Supporting Information

\title{
Radically-initiated group transfer polymerization of methacrylates by titanium amino-phenolate complexes
}

Daniel L. Coward, Benjamin R. M. Lake, Rinaldo Poli and Michael P. Shaver*

EaStCHEM School of Chemistry, University of Edinburgh, Edinburgh, EH9 3FJ, UK.

CNRS, LCC (Laboratoire de Chimie de Coordination) and Université de Toulouse, UPS, INPT, 205 route de Narbonne, BP 44099, 31077 Toulouse Cedex 4, France.

School of Materials, University of Manchester, Oxford Road, Manchester, M13 9PL

\section{CONTENTS}

\section{MATERIALS AND METHODS}

\section{SYNTHETIC PROCEDURES}

\section{COMPLEX SYNTHESIS}

Scheme S1 Synthesis of ligand precursors Lig1 $\mathrm{H}_{2}-\mathrm{Lig}_{\mathrm{H}} \mathrm{H}_{3}$

Scheme S2 a) Synthesis of complexes 1-5 and b) synthesis of complexes 6 and 7

\section{POLYMERIZATION DATA}

Table S1 Polymerization screening of MMA with complexes 1-7

Table S2 Polymerization of MMA, with and without 1 and radical scavenger dihydroanthracene

Table S3 Polymerization solvent screening of MMA with complexes 1-3

Table S4 Additional polymerization screening

\section{KINETIC DATA}

Figure $\mathbf{S 1} \quad$ MMA kinetics with complex 1

Figure S2 MMA kinetics, with complex 1, using different solvents

Figure S3 MMA kinetics, with and without complex 1, using different concentrations of initiator

Figure S4 MMA kinetics, with complex 1, using different concentrations of initiator

Figure S5 Addition of extra 1 during MMA polymerization

Figure S6 Use of a macroinitiator, with and without added 1

Figure S7 $\quad$ nBuMA kinetics with complex 1

Figure S8 MMA kinetics using different concentrations of complex 1

Figure S9 MMA kinetics with complex 1 and $\mathbf{4}$

\section{MECHANISTIC PATHWAYS}

Scheme S3 Energy diagram showing the relative stability of 6-coordinate titanium species, relative to the 5-coordinate complex 
Scheme S5 Energy diagram showing the proposed monometallic group transfer polymerization mechanism

\section{MOLECULAR STRUCTURES}

Figure S10 Molecular structures of 2, 3 and 4

Figure S11 Molecular structures of $2(\mu-\mathrm{Cl})_{2}$ and $\mathbf{3}(\mu-\mathrm{Cl})_{2}$

Figure S12 Dimeric structure of complex 7

\section{NMR SPECTRA}

Figure S13 $\quad{ }^{1} \mathrm{H}$ NMR spectrum of complex $2(\boldsymbol{\mu}-\mathrm{Cl})_{2}\left(500 \mathrm{MHz}, \mathrm{C}_{6} \mathrm{D}_{6}\right)$

Figure S14 $\quad{ }^{1} \mathrm{H}$ NMR spectrum of complex $3(\mu-\mathrm{Cl})_{2}\left(500 \mathrm{MHz}, \mathrm{C}_{6} \mathrm{D}_{6}\right)$

Figure S15 $\quad{ }^{1} \mathrm{H}$ NMR spectrum of complex $6\left(500 \mathrm{MHz}, \mathrm{C}_{6} \mathrm{D}_{6}\right)$

Figure S16 $\quad{ }^{1} \mathrm{H}$ NMR spectrum of complex $7\left(500 \mathrm{MHz}, \mathrm{C}_{6} \mathrm{D}_{6}\right)$

Figure S17 $\quad{ }^{13} \mathrm{C}\left\{{ }^{1} \mathrm{H}\right\}$ NMR spectrum of complex $7\left(126 \mathrm{MHz}, \mathrm{C}_{6} \mathrm{D}_{6}\right)$

Figure S18 Variable temperature ${ }^{1} \mathrm{H}$ NMR spectra of complex $7\left(400 \mathrm{MHz}, \mathrm{C}_{6} \mathrm{D}_{6}\right)$

Figure S19 Variable temperature ${ }^{1} \mathrm{H}$ NMR spectra of complex 7 (400 MHz, d8-toluene)

Figure S20 Determination of tacticity of poly (methyl methacrylate) $\left(\mathrm{CDCl}_{3}\right)$

Figure S21 $\quad{ }^{1} \mathrm{H}$ NMR spectrum of purified poly(methyl methacrylate) produced by complex 7 (500 $\mathrm{MHz}$, $\mathrm{CDCl}_{3}$ )

Figure S22 Overlaid ${ }^{1} \mathrm{H}$ NMR spectra of pure complex 1 (bottom) and product of reaction between complex 1 and $\mathrm{V}-70\left(80^{\circ} \mathrm{C}, 30\right.$ mins) (bottom) $\left(600 \mathrm{MHz}, \mathrm{C}_{6} \mathrm{D}_{6}\right)$

Figure S23 Overlaid ${ }^{1} \mathrm{H}$ NMR spectra of complex 1 and V-601 (bottom) and product of reaction between complex 1 and $\mathrm{V}-601\left(80^{\circ} \mathrm{C}, 20\right.$ hours) (top) (500 MHz, $\mathrm{C}_{6} \mathrm{D}_{6}$ )

\section{X-RAY CRYSTALLOGRAPHIC DATA}

Figure S24 Molecular structure of $\mathbf{2}$

Figure S25 Molecular structure of $\mathbf{2}(\mu-\mathrm{Cl})_{2}$

Figure S26 Molecular structure of 3

Figure S27 Molecular structure of $\mathbf{3}(\boldsymbol{\mu}-\mathrm{Cl})_{2}$

Figure S28 Molecular structure of 4

Figure S29 Molecular structure of 6

Figure S30 Molecular structures of 6 viewed along the N-Ti bond illustrating the a) major and b) minor components of the disorder and $\mathbf{c}$ ) the superimposition of both components

\section{COMPUTATIONAL DATA}

Table S5 Benchmarking of three functionals against published crystal structure

Cartesian coordinates and energies for all optimized geometries

\section{NOTES AND REFERENCES}




\section{MATERIALS AND METHODS}

All experiments involving moisture- and air-sensitive compounds were performed under a nitrogen atmosphere using an MBraun LABmaster sp glovebox system equipped with a $-35^{\circ} \mathrm{C}$ freezer and $\left[\mathrm{H}_{2} \mathrm{O}\right]$ and $\left[\mathrm{O}_{2}\right]$ analysers or using standard Schlenk techniques. Solvents used were obtained from a solvent purification system (Innovative Technologies) consisting of columns of alumina and copper catalyst and were further degassed by three freeze-pump-thaw cycles prior to use. Benzene- $d_{6}$ and THF- $d_{8}$ were dried by stirring over sodium/benzophenone, before being collected by distillation and degassed by three freeze-pump-thaw cycles. Chloroform- $d_{1}$ was used as received. Methyl methacrylate (MMA), methyl acrylate (MA), tert-butyl methacrylate ( $\left.{ }^{t} \mathrm{BuMA}\right)$ and $n$-butyl methacrylate ( $\left.{ }^{n} \mathrm{BuMA}\right)$ were dried by stirring over calcium hydride for a minimum of 24 hours, before being vacuum transferred and stored at $-35^{\circ} \mathrm{C}$. V-70 (Wako) was suspended in dry acetone at $10^{\circ} \mathrm{C}$, stirred vigorously for ca. 30 mins before collection by filtration and drying in vacuo followed by storage at $-35^{\circ} \mathrm{C}$ under an inert atmosphere. Gel permeation chromatography (GPC) was carried out in THF at a flow rate of $1 \mathrm{~mL} \mathrm{~min}{ }^{-1}$ at $35^{\circ} \mathrm{C}$ on a Malvern Instruments Viscotek 270 GPC Max triple detection system with $2 \times$ mixed bed styrene/DVB columns $(300 \times 7.5 \mathrm{~mm})$. Absolute molar masses were obtained using $\mathrm{dn} / \mathrm{dc}$ values of 0.088 for poly(methyl methacrylate), ${ }^{1} 0.068$ for poly(methyl acrylate),,$^{2} 0.076$ for poly( $n$-butyl methacrylate), ${ }^{3}$ and 0.185 for poly(styrene). ${ }^{4} \mathrm{NMR}$ spectra were obtained on either a $400 \mathrm{MHz}, 500 \mathrm{MHz}$ or $600 \mathrm{MHz}$ Bruker Avance III spectrometer. Solution magnetic moments were determined via NMR spectroscopy using Evans' method. ${ }^{5}$ Elemental analyses were performed by Stephen Boyer at London Metropolitan University.

\section{GENERAL POLYMERIZATION PROCEDURE}

In a glovebox, a small ampoule was charged with titanium(III) complex (24.0 $\mu \mathrm{mol})$, monomer $(2.40 \mathrm{mmol})$, solvent (solvent:monomer, 1:1 v/v) and V-70 $(24.0 \mu \mathrm{mol})$. The ampoule was brought out of the glovebox and heated at $80^{\circ} \mathrm{C}$ for $x$ hours with a stir-rate of $500 \mathrm{rpm}$. After this time, the ampoule was cooled rapidly to ambient temperature, and an aliquot removed for analysis by ${ }^{1} \mathrm{H}$ NMR spectroscopy to determine monomer conversion. The remainder of the reaction mixture was taken-up in a small volume of THF (ca. $2 \mathrm{ml}$ ), and the polymer precipitated by addition of the THF solution to acidified methanol $\left(\mathrm{MeOH}: \mathrm{HCl}_{(\mathrm{aq})}\right.$, ca. $\left.75 \mathrm{ml}: 1 \mathrm{ml}\right)$. The polymer was collected by filtration and dried in vacuo.

\section{REPRESENTATIVE POLYMERIZATION PROCEDURE}

${ }^{\mathrm{t}} \mathrm{Bu},{ }^{\mathrm{t}} \mathrm{Bu}, \mathrm{NMe}_{2}\left[\mathrm{O}_{2} \mathrm{NN}^{\prime}\right]-\mathrm{TiCl}, 1$ (0.014 g, $\left.0.02 \mathrm{mmol}\right), \mathrm{V}-70(0.006 \mathrm{~g}, 0.02 \mathrm{mmol})$, methyl methacrylate $(0.200 \mathrm{~g}, 2$ $\mathrm{mmol})$ and toluene $(0.185 \mathrm{~g}, 1: 1 \mathrm{v} / \mathrm{v})$ were added to an ampule containing a microstirrer bar under inert atmosphere, which was then sealed and heated at $80{ }^{\circ} \mathrm{C}$ with stirring for $1 \mathrm{~h} .{ }^{1} \mathrm{H}$ NMR spectroscopic analysis of the crude residue indicated $88 \%$ monomer conversion. Precipitation into acidified methanol gave white poly(methyl methacrylate), with $\mathrm{M}_{\mathrm{n}}=37800$ and $€=1.13$.

\section{CRYSTALLOGRAPHY}

X-Ray diffraction data was collected on an Agilent SuperNova diffractometer fitted with an Atlas CCD detector with Mo-K $K_{\alpha}$ radiation $(\lambda=0.7107 \AA)$ or $\mathrm{Cu}-\mathrm{K}_{\alpha}$ radiation $(\lambda=1.5418 \AA$ ). Crystals were mounted under paratone on MiTeGen loops. The structures were solved by direct methods using SHELXS or SHELXT interfaced through Olex 2 and refined by full-matrix least-squares on F2 using SHELXL, interfaced through Olex2. ${ }^{6-8}$ Molecular graphics for all structures were generated using POV-RAY, POVLabel and Ortep.

\section{COMPUTATIONAL METHODS}

The computational work was carried out using the Gaussian09.E01 suite of programs. ${ }^{9}$ The geometry optimizations were performed without any symmetry constraint using the M06, B3LYP and BP86 functionals. The SVP basis functions were used for all atoms. The unrestricted formulation was used for all open-shell molecules, yielding only minor spin contamination. Maximum deviations for $\left\langle\mathrm{S}^{2}\right\rangle$ at convergence were 0.766 (vs the theoretical value of 0.75 ) for spin doublets and 2.013 (vs 2.00) for triplets. 


\section{SYNTHETIC PROCEDURES}

Synthesis of ${ }^{\mathrm{t}-\mathrm{Bu}, \mathrm{t}-\mathrm{Bu}, \mathrm{NMe} 2}\left[\mathrm{O}_{2} \mathrm{NN}^{\prime}\right] \mathrm{TiCl}(\mathrm{THF})(\mathbf{1}) .{ }^{10}$ A solution of $\mathrm{Lig} \mathrm{H}_{2}(0.60 \mathrm{~g}, 1.14 \mathrm{mmol})$ and Li(HMDS) (0.38 g, $2.27 \mathrm{mmol}$ ) in THF $(5 \mathrm{ml})$ was stirred at ambient temperature for 30 minutes. To this was added a suspension of $\mathrm{TiCl}_{3}(\mathrm{THF})_{3}(0.42 \mathrm{~g}, 1.14 \mathrm{mmol})$ in THF $(5 \mathrm{ml})$. The resulting mixture was stirred for 4 hours. After this time, the volatiles were removed in vacuo, and the residue taken-up in to toluene $(15 \mathrm{ml})$, filtered through Celite and the solvent removed in vacuo to yield a pale yellow crystalline solid ( $0.62 \mathrm{~g}, 0.92 \mathrm{mmol}, 80 \%$ ). Meff (Evans' Method, $\left.\mathrm{C}_{6} \mathrm{D}_{6}\right)=1.7 \mu_{\mathrm{B}}$.

Synthesis of ${ }^{\mathrm{Me}, \mathrm{Me}, \mathrm{NMe} 2}\left[\mathrm{O}_{2} \mathrm{NN}^{\prime}\right] \mathrm{TiCl}(\mathrm{THF})$ (2). A solution of $\mathrm{Lig}_{2} \mathrm{H}_{2}(0.20 \mathrm{~g}, 0.56 \mathrm{mmol})$ and Li(HMDS) (0.19 g, 1.12 $\mathrm{mmol})$ in toluene $(10 \mathrm{ml})$ was stirred at ambient temperature for 30 minutes. To this was added a suspension of $\mathrm{TiCl}_{3}(\mathrm{THF})_{3}(0.21 \mathrm{~g}, 0.56 \mathrm{mmol})$ in THF $(5 \mathrm{ml})$. The resulting mixture was stirred for 24 hours. After this time, the volatiles were removed in vacuo, and the residue taken-up in to toluene $(15 \mathrm{ml})$, filtered through Celite and the solvent removed in vacuo. Recrystallization of the complex from THF/n-hexane at $-35{ }^{\circ} \mathrm{C}$ produced the pure complex as pale yellow crystals $(0.26 \mathrm{~g}, 0.51 \mathrm{mmol}, 91 \%)$. Single crystals of 2 suitable for $x$-ray diffraction analysis could be obtained by the vapour diffusion of $n$-hexane in to a concentrated THF solution. Single crystals of $\mathbf{2}(\boldsymbol{\mu}-$ $\mathrm{Cl}_{2}$ could be obtained on prolonged standing of a concentrated solution of 2 in $\mathrm{C}_{6} \mathrm{D}_{6}{ }^{1} \mathrm{H} \mathrm{NMR}\left(500 \mathrm{MHz}, \mathrm{C}_{6} \mathrm{D}_{6}\right) \delta$ 6.99 (s), 6.59 (s), 4.60 (br s), 3.44 (br s), 2.33 (s), 2.22 (s), 2.16 (br s), 1.79 (br s) ppm. $\mu_{\text {eff }}$ (Evans' Method, ds-THF) $=1.6 \mu_{\mathrm{B}}$. Analysis Calculated for $\mathrm{C}_{26} \mathrm{H}_{38} \mathrm{ClN}_{2} \mathrm{O}_{3} \mathrm{Ti}: \mathrm{C}, 61.24 ; \mathrm{H}, 7.51 ; \mathrm{N}, 5.49$. Found: $\mathrm{C}, 61.09 ; \mathrm{H}, 7.61 ; \mathrm{N}, 5.41$.

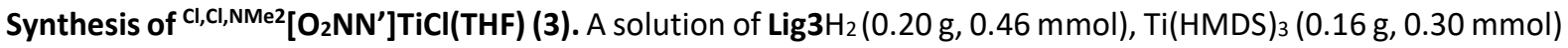
and $\mathrm{TiCl}_{3}(\mathrm{THF})_{3}(0.056 \mathrm{~g}, 0.15 \mathrm{mmol})$ in THF $(5 \mathrm{ml})$ was stirred at ambient temperature for 1 hour. After this time, the volatiles were removed in vacuo, and the residue taken-up in to a minimum of THF. Precipitation with an excess of $n$-hexane gave the pure product as a pale yellow crystalline solid $(0.19 \mathrm{~g}, 0.32 \mathrm{mmol}, 70 \%) .{ }^{1} \mathrm{H} \mathrm{NMR}$ $\left(500 \mathrm{MHz}, \mathrm{C}_{6} \mathrm{D}_{6}\right) \delta 7.35$ (s), 7.11* (s), 6.60 (s), 6.43* (s), 4.18 (br s), 2.99 (br s), 2.54* (br s), 2.21 (br s), 1.81 (br s), 1.65 (br s) ppm. $\mu_{\text {eff }}$ (Evans' Method, d8-THF) = $1.7 \mu$ в. Analysis Calculated for $\mathrm{C}_{22} \mathrm{H}_{26} \mathrm{Cl}_{5} \mathrm{~N}_{2} \mathrm{O}_{3} \mathrm{Ti}: \mathrm{C}, 44.67 ; \mathrm{H}, 4.43$; $\mathrm{N}$, 4.74. Found: $\mathrm{C}, 44.59 ; \mathrm{H}, 4.56 ; \mathrm{N}, 4.75$.

Synthesis of ${ }^{\mathrm{t}-\mathrm{Bu}, \mathrm{t}-\mathrm{Bu}, \mathrm{THF}}\left[\mathrm{O}_{2} \mathrm{NO}^{\prime}\right] \mathrm{TiCl}(\mathrm{THF})$ (4). A solution of $\mathrm{Lig}_{4} \mathrm{H}_{2}(0.20 \mathrm{~g}, 0.37 \mathrm{mmol})$ and Li(HMDS) $(0.12 \mathrm{~g}, 0.74$ $\mathrm{mmol})$ in toluene $(10 \mathrm{ml})$ was stirred at ambient temperature for 30 minutes. To this was added a suspension of $\mathrm{TiCl}_{3}(\mathrm{THF})_{3}(0.14 \mathrm{~g}, 0.37 \mathrm{mmol})$ in THF $(5 \mathrm{ml})$. The resulting mixture was stirred for 24 hours. After this time, the volatiles were removed in vacuo, and the residue taken-up in to toluene $(15 \mathrm{ml})$, filtered through Celite and the solvent removed in vacuo. Recrystallization of the complex from toluene/ $n$-hexane at reflux produced the pure complex as a pale yellow crystalline solid $(0.22 \mathrm{~g}, 0.31 \mathrm{mmol}, 84 \%)$. Single crystals of 4 suitable for $\mathrm{x}$-ray diffraction analysis could be obtained by the vapour diffusion of $n$-hexane in to a concentrated toluene solution. $\mu_{\text {eff }}$ (Evans' Method, $\left.\mathrm{C}_{6} \mathrm{D}_{6}\right)=1.7 \mu_{\mathrm{B}}$. Analysis Calculated for $\mathrm{C}_{39} \mathrm{H}_{61} \mathrm{ClNO}_{4} \mathrm{Ti}: \mathrm{C}, 67.77 ; \mathrm{H}, 8.90 ; \mathrm{N}, 2.03$. Found: $\mathrm{C}$, 67.89; H, 9.01; N, 2.14.

Synthesis of ${ }^{\mathrm{t}-\mathrm{Bu}, \mathrm{t}-\mathrm{Bu}, \mathrm{Bn}}\left[\mathrm{O}_{2} \mathrm{~N}_{2}\right] \mathrm{TiCl}(\mathrm{THF})$ (5). A solution of $\mathrm{Lig} 5 \mathrm{H}_{2}(0.20 \mathrm{~g}, 0.30 \mathrm{mmol})$ and $\mathrm{Li}(\mathrm{HMDS})(0.10 \mathrm{~g}, 0.59$ $\mathrm{mmol})$ in toluene $(10 \mathrm{ml})$ was stirred at ambient temperature for 30 minutes. To this was added a suspension of $\mathrm{TiCl}_{3}(\mathrm{THF})_{3}(0.11 \mathrm{~g}, 0.30 \mathrm{mmol})$ in THF $(5 \mathrm{ml})$. The resulting mixture was stirred for 24 hours. After this time, the volatiles were removed in vacuo, and the residue taken-up in to toluene (15 $\mathrm{ml})$, filtered through Celite and the solvent removed in vacuo. Recrystallization of the complex from toluene/ $n$-hexane at reflux produced the pure complex as a pale yellow crystalline solid $\left(0.24 \mathrm{~g}, 0.29 \mathrm{mmol}, 97 \%\right.$ ). $\mu_{\text {eff }}$ (Evans' Method, $\mathrm{C}_{6} \mathrm{D}_{6}$ ) $=1.5 \mu_{\mathrm{B}}$. Analysis Calculated for $\mathrm{C}_{50} \mathrm{H}_{70} \mathrm{ClN}_{2} \mathrm{O}_{3} \mathrm{Ti}: \mathrm{C}, 72.32 ; \mathrm{H}, 8.50 ; \mathrm{N}, 3.37$. Found: $\mathrm{C}, 67.62 ; \mathrm{H}, 8.41 ; \mathrm{N}, 3.36 .^{11}$

Synthesis of t-Bu,t-Bu$\left[\mathrm{O}_{3} \mathrm{~N}\right] \mathrm{Ti}(\mathrm{THF})$ (6). A solution of $\mathrm{Lig6H}_{3}(0.32 \mathrm{~g}, 0.48 \mathrm{mmol})$ and $\mathrm{Li}(\mathrm{HMDS})(0.24 \mathrm{~g}, 1.43 \mathrm{mmol})$ in toluene was stirred at ambient temperature for 2 hours. Solid $\mathrm{TiCl}_{3}(\mathrm{THF})_{3}(0.18 \mathrm{~g}, 0.48 \mathrm{mmol})$ was then added and the resulting suspension was stirred at ambient temperature for 18 hours. After this time, the volatiles were removed in vacuo, and the residue taken-up in to toluene $(15 \mathrm{ml})$, filtered through Celite and the solvent removed in vacuo. The resulting off-white solid was washed with $n$-hexane $(2 \times 10 \mathrm{ml})$ and dried in vacuo (0.33 g, $0.42 \mathrm{mmol}, 89$ \%). ${ }^{1} \mathrm{H}$ NMR (500 MHz, $\mathrm{C}_{6} \mathrm{D}_{6}$ ) $\delta 7.40$ (br s), 5.26 (br s), 4.86 (br s), 4.65 (s), 1.58 (br s), 1.34 (s) ppm. $\mu_{\text {eff }}$ (Evans' Method, $\left.\mathrm{C}_{6} \mathrm{D}_{6}\right)=1.6 \mu_{\mathrm{B}}$. Analysis Calculated for $\mathrm{C}_{49} \mathrm{H}_{74} \mathrm{NO}_{4} \mathrm{Ti}: \mathrm{C}, 74.59 ; \mathrm{H}, 9.45 ; \mathrm{N}, 1.78$. Found: C, 68.99; H, 9.12; N, 2.04. ${ }^{11}$ 
Synthesis of ${ }^{\mathrm{Me}, \mathrm{Me}}\left[\mathrm{O}_{3} \mathrm{~N}\right] \mathrm{Ti}(7) .{ }^{12} \mathrm{~A}$ solution of $\mathrm{Lig} \mathrm{H}_{3}(0.20 \mathrm{~g}, 0.48 \mathrm{mmol})$ and Li(HMDS) $(0.24 \mathrm{~g}, 1.43 \mathrm{mmol})$ in toluene was stirred at ambient temperature for 2 hours. Solid $\mathrm{TiCl}_{3}(\mathrm{THF})_{3}(0.18 \mathrm{~g}, 0.48 \mathrm{mmol})$ was then added and the resulting suspension was stirred at ambient temperature for 18 hours. After this time, the volatiles were removed in vacuo, and the residue taken-up in to toluene $(15 \mathrm{ml})$, filtered through Celite and the solvent removed in vacuo. The resulting violet solid was washed with $n$-hexane $(2 \times 10 \mathrm{ml})$ and dried in vacuo $(0.18 \mathrm{~g}$, $0.39 \mathrm{mmol}, 82 \%) .{ }^{1} \mathrm{H}$ NMR $\left(500 \mathrm{MHz}, \mathrm{C}_{6} \mathrm{D}_{6}\right) \delta 6.80(\mathrm{~s}, 3 \mathrm{H}, \mathrm{ArH}), 6.61(\mathrm{~d}, \mathrm{~J}=1.8 \mathrm{~Hz}, 1 \mathrm{H}, \mathrm{ArH}), 6.53(\mathrm{~s}, 2 \mathrm{H}, \mathrm{ArH})$, 3.70 (v. br s), 3.04 (br s), 2.19 (s, 9H, CH $), 2.17\left(\mathrm{~s}, 6 \mathrm{H}, \mathrm{CH}_{3}\right), 2.11\left(\mathrm{~s}, 3 \mathrm{H}, \mathrm{CH}_{3}\right)$ ppm. ${ }^{13} \mathrm{C}\left({ }^{1} \mathrm{H}\right) \mathrm{NMR}\left(126 \mathrm{MHz} \mathrm{C}_{6} \mathrm{D}_{6}\right)$ $\delta$ 133.0, 131.6, 130.1, 129.9, 127.4, 126.0, 125.0, 123.9, 123.4, 60.2, 59.7, 20.8, 20.5, 19.3, 16.5 ppm. Meff (Evans' Method, $\left.\mathrm{C}_{6} \mathrm{D}_{6}\right)=0 \mu_{\text {в }}$ (diamagnetic). Analysis Calculated for $\mathrm{C}_{27} \mathrm{H}_{30} \mathrm{NO}_{3} \mathrm{Ti}_{2} 2 \mathrm{H}_{2} \mathrm{O}: \mathrm{C}, 64.80 ; \mathrm{H}, 6.85 ; \mathrm{N}, 2.80$. Found: C, 64.34; H, 6.90; N, 2.85. 


\section{COMPLEX SYNTHESIS}

Scheme S1 Synthesis of ligand precursors Lig1 $\mathrm{H}_{2}-\mathrm{Lig}_{3} \mathrm{H}_{3}$

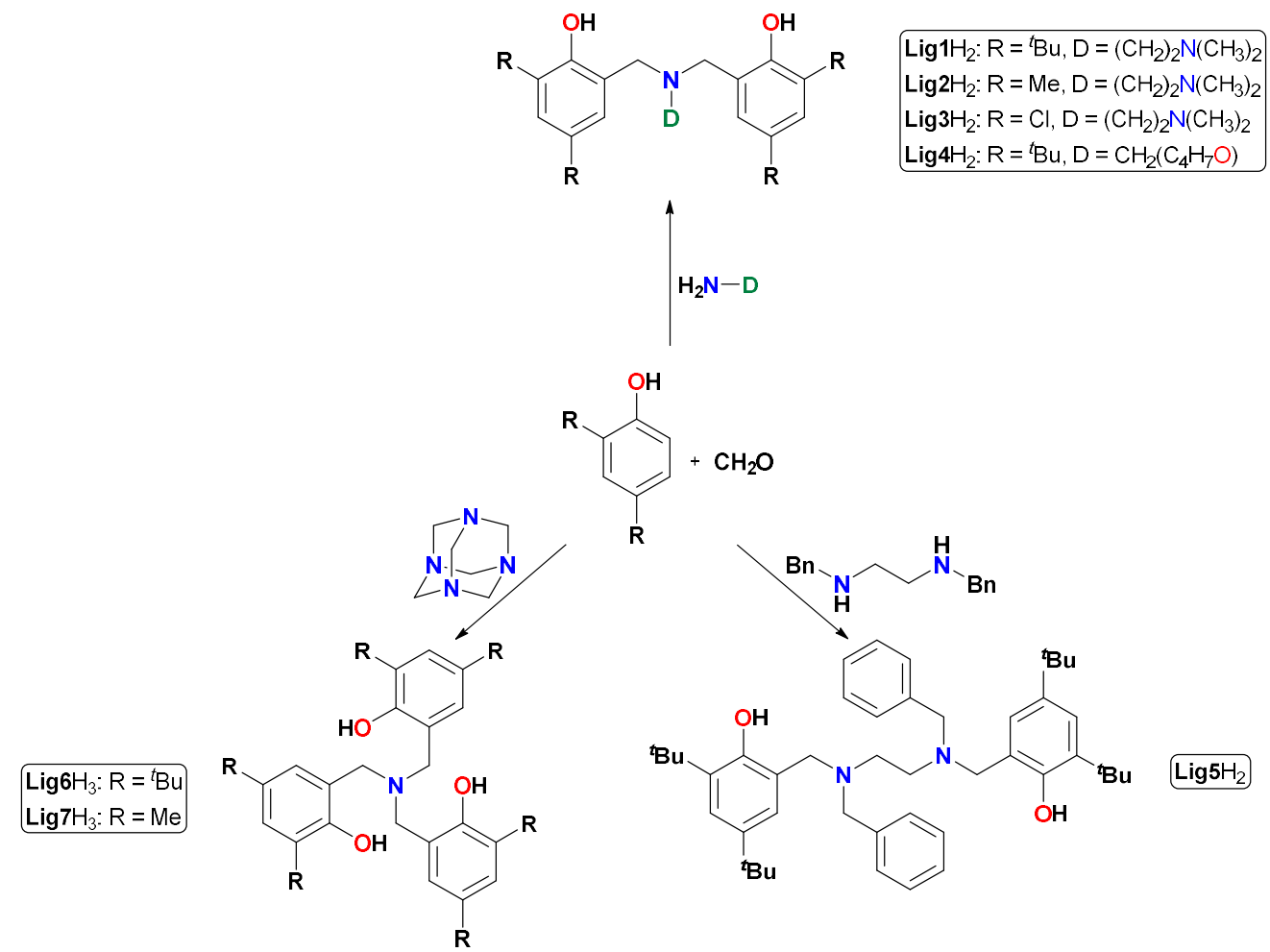

Scheme S2a) Synthesis of complexes 1-5 and b) synthesis of complexes $\mathbf{6}$ and $\mathbf{7}$

a)

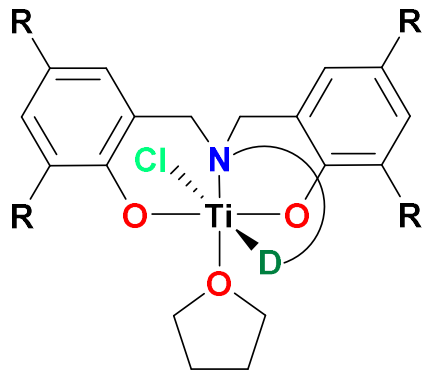

1: $\mathrm{R}={ }^{\mathrm{t}} \mathrm{Bu}, \mathrm{D}=\left(\mathrm{CH}_{2}\right)_{2} \mathrm{~N}\left(\mathrm{CH}_{3}\right)_{2}$

2: $\mathrm{R}=\mathrm{Me}, \mathrm{D}=\left(\mathrm{CH}_{2}\right)_{2} \mathrm{~N}\left(\mathrm{CH}_{3}\right)_{2}$

$\begin{array}{ll}\text { Lig1H }_{2} & 1 / 3 \mathrm{TiCl}_{3}(\mathrm{THF})_{3} \\ \text { Lig2 }_{2} & \stackrel{2}{3} \mathrm{Ti}(\mathrm{HMDS})_{3} \\ \longrightarrow\end{array}$

3: $\mathrm{R}=\mathrm{Cl}, \mathrm{D}=\left(\mathrm{CH}_{2}\right)_{2} \mathrm{~N}\left(\mathrm{CH}_{3}\right)_{2}$

4: $\mathrm{R}={ }^{t} \mathrm{Bu}, \mathrm{D}=\mathrm{CH}_{2}\left(\mathrm{C}_{4} \mathrm{H}_{7} \mathrm{O}\right)$

Lig3 $\mathrm{H}_{2}$

Lig4 $\mathrm{H}_{2}$

i) $2 \mathrm{Li}(\mathrm{HMDS})$

Lig5 $\mathrm{H}_{2}$

ii) $\mathrm{TiCl}_{3}(\mathrm{THF})_{3} \quad t_{\mathrm{Bu}}$

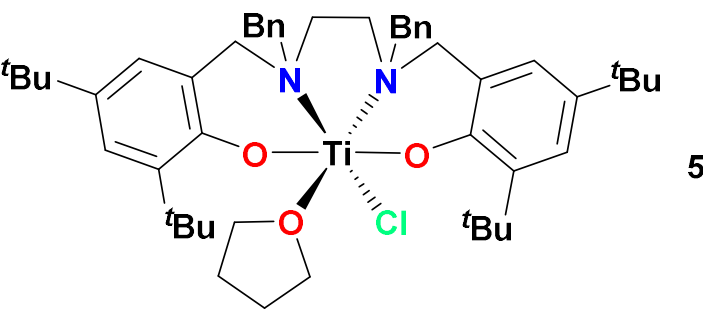

b)

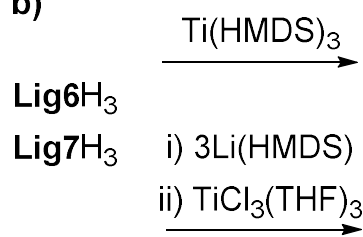

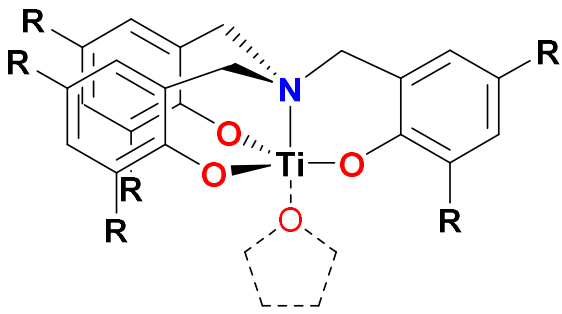

6: $R={ }^{t} \mathrm{Bu}$

7: $R=M e$ 
POLYMERIZATION DATA

Table S1 Polymerization screening of MMA with complexes 1-7

\begin{tabular}{|c|c|c|c|c|c|c|}
\hline Entry & Complex & Conv. (\%) & $M_{n, t h}(D a)$ & $M_{n}(D a)$ & $\bigoplus$ & mm:rm:rr \\
\hline$S 1^{c}$ & 1 & 50 & 5000 & 19700 & 1.10 & $15: 47: 38$ \\
\hline$S 2^{c}$ & 2 & 97 & 9700 & 46500 & 1.61 & $6: 39: 55$ \\
\hline $\mathbf{S 3}^{\mathrm{b}}$ & 3 & 73 & 7300 & 20700 & 1.59 & $6: 39: 55$ \\
\hline$S 4^{b}$ & 4 & 80 & 8000 & 21600 & 1.26 & $18: 48: 34$ \\
\hline$S 5^{b}$ & 5 & 55 & 5500 & 16900 & 1.97 & $8: 34: 57$ \\
\hline$S 6^{c}$ & 6 & 76 & 7600 & 19100 & 2.41 & $8: 38: 54$ \\
\hline$S 7^{c}$ & 7 & 70 & 7000 & 29700 & 2.01 & $4: 36: 59$ \\
\hline
\end{tabular}

a Conditions: [MMA]:[Ti(III)]:[V-70] = 100:1.0:1.0, MMA:toluene $=1: 1(\mathrm{v} / \mathrm{v}) .80^{\circ} \mathrm{C} .{ }^{\mathrm{b}} 1$ hour. ${ }^{\mathrm{c}} 2.5$ hours. Conversion determined by ${ }^{1} \mathrm{H}$ NMR spectroscopy. $\mathrm{M}_{\mathrm{n}, \mathrm{th}}=[\mathrm{MMA}]_{0} /[\mathrm{Ti}(\mathrm{III})]_{0} \times \mathrm{M}(\mathrm{MMA}) \times$ conversion.

Table S2 Polymerization of MMA, with and without complex 1 and radical scavenger dihydroanthracene (DHA) ${ }^{\mathrm{a}}$

\begin{tabular}{cccc}
\hline Entry & Equiv. Ti & Equiv. DHA & Conv. (\%) \\
\hline S8 & 0 & 0 & 56 \\
S9 & 0 & 5 & 33 \\
S10 & 0 & 20 & 20 \\
S11 & 1 & 0 & 84 \\
S12 & 1 & 5 & 81 \\
\hline
\end{tabular}

${ }^{a}$ Conditions: [MMA]:[1]:[V-70]:[DHA] = 100:x:1.0:y, MMA:toluene = 1:1 (v/v), 80 $\mathrm{C}, 40 \mathrm{~min}$. DHA is added at the start of the polymerization. Conversion determined by ${ }^{1} \mathrm{H}$ NMR spectroscopy. DHA is slow to react, and therefore does not quench the primary radicals of $\mathrm{V}-70$. This accounts for the suppression of the free-radical polymerization, but the absence of complete termination. 
Table S3 Polymerization solvent screening of MMA with complexes $\mathbf{1 - 3}^{\mathrm{a}}$

\begin{tabular}{ccccccc}
\hline Entry & Complex & Solvent & Conv. (\%) & $\mathbf{M}_{\mathbf{n}, \text { th }}(\mathbf{D a})$ & $\mathbf{M}_{\mathbf{n}}(\mathbf{D a})$ & $\boldsymbol{\Xi}$ \\
\hline S13 & 1 & toluene & 93 & 9300 & 54800 & 1.19 \\
S14 & 2 & toluene & $>99$ & 10000 & 95300 & 1.48 \\
S15 & 3 & toluene & 76 & 7600 & 22800 & 1.40 \\
S16 & 1 & 1,4-dioxane & 32 & 3200 & 29400 & 1.13 \\
S17 & 2 & 1,4-dioxane & $>99$ & 10000 & 85800 & 1.54 \\
S18 & 3 & 1,4-dioxane & 66 & 6600 & 22700 & 1.53 \\
S19 & 1 & THF & 12 & 1200 & 15500 & 1.25 \\
S20 & 2 & THF & $>99$ & 10000 & 68600 & 1.60 \\
S21 & 3 & THF & 54 & 5400 & 23500 & 1.54 \\
\hline
\end{tabular}

${ }^{a}$ Conditions: $[\mathrm{MMA}]:[\mathrm{Ti}(\mathrm{III})]:[\mathrm{V}-70]=100: 1.0: 0.5$, MMA:solvent $=1: 1(\mathrm{v} / \mathrm{v}), 75{ }^{\circ} \mathrm{C}, 2.5$ hours. Conversion determined by ${ }^{1} \mathrm{H}$ NMR spectroscopy. $\mathrm{Mn}_{\mathrm{n}, \mathrm{th}}=[\mathrm{MMA}]_{0} /[\mathrm{Ti}(\mathrm{III})]_{0} \times \mathrm{M}(\mathrm{MMA}) \times$ conversion.

Table S4 Additional polymerization screening ${ }^{\mathrm{a}}$

\begin{tabular}{ccccccc}
\hline Entry & Complex & Monomer & Conv. (\%) & $\mathbf{M}_{n, \text { th }}$ (Da) & $\mathbf{M n}_{\mathbf{n}}$ (Da) & $\boldsymbol{\Xi}$ \\
\hline S22 & 1 & MA & 81 & 7000 & 23800 & 2.68 \\
S23 & 2 & ${ }^{t}$ BuMA & $<1$ & - & - & - \\
S24 & 1 & ${ }^{t}$ BuMA & $<1$ & - & - & - \\
S25 & 2 & ${ }^{n}$ BuMA & $>99$ & 14200 & 45000 & 2.12 \\
S26 & 1 & ${ }^{n}$ BuMA & 81 & 11500 & 58500 & 1.14 \\
S27 $^{\text {b }}$ & 1 & MMA & 80 & 8000 & 30000 & 1.09 \\
\hline
\end{tabular}

${ }^{a}$ Conditions: [Monomer]:[Ti(III)]:[V-70] = 100:1.0:1.0, monomer:toluene $=1: 1(\mathrm{v} / \mathrm{v}) .80^{\circ} \mathrm{C} .2 .5$ hours. ${ }^{\mathrm{b}}$ No added solvent. Conversion determined by ${ }^{1} \mathrm{H}$ NMR spectroscopy. $\mathrm{Mn}_{\mathrm{n}, \mathrm{th}}=[\text { monomer }]_{0} /[\mathrm{Ti}(\mathrm{III})]_{0} \times \mathrm{M}($ monomer $) \times$ conversion. 


\section{KINETIC DATA}

Figure S1 MMA kinetics with complex 1
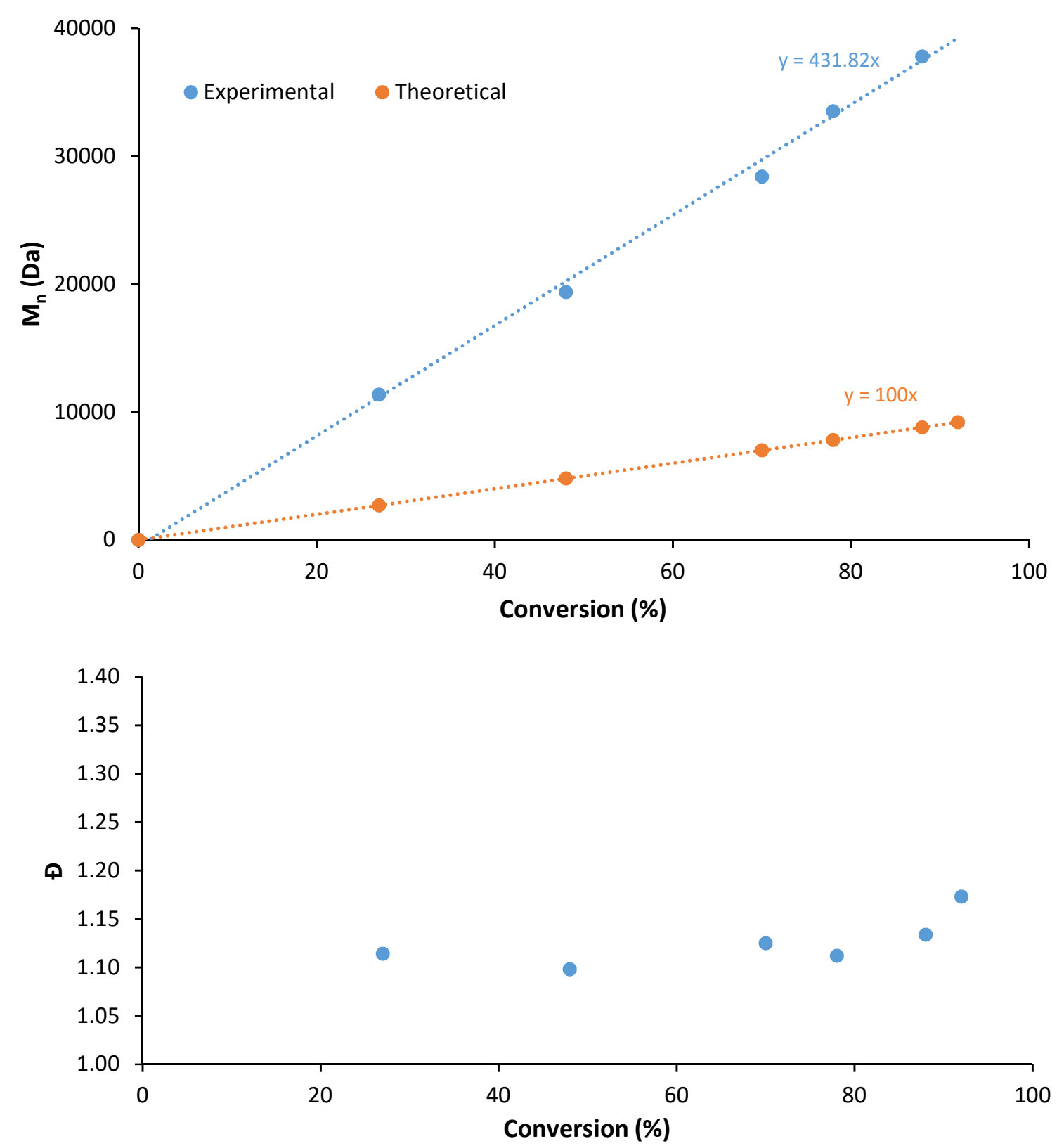

Conditions: $[\mathrm{MMA}]:[\mathbf{1}]:[\mathrm{V}-70]=100: 1.0: 1.0, \mathrm{MMA}:$ toluene $=1: 1(\mathrm{v} / \mathrm{v}), 80^{\circ} \mathrm{C}$. Conversion determined by ${ }^{1} \mathrm{H}$ NMR spectroscopy. $\mathrm{M}_{\mathrm{n}, \mathrm{th}}=[\mathrm{MMA}]_{0} /[\mathrm{Ti}(\mathrm{III})]_{0} \times \mathrm{M}(\mathrm{MMA}) \times$ conversion. 
Figure S2 MMA kinetics, with complex 1, using different solvents

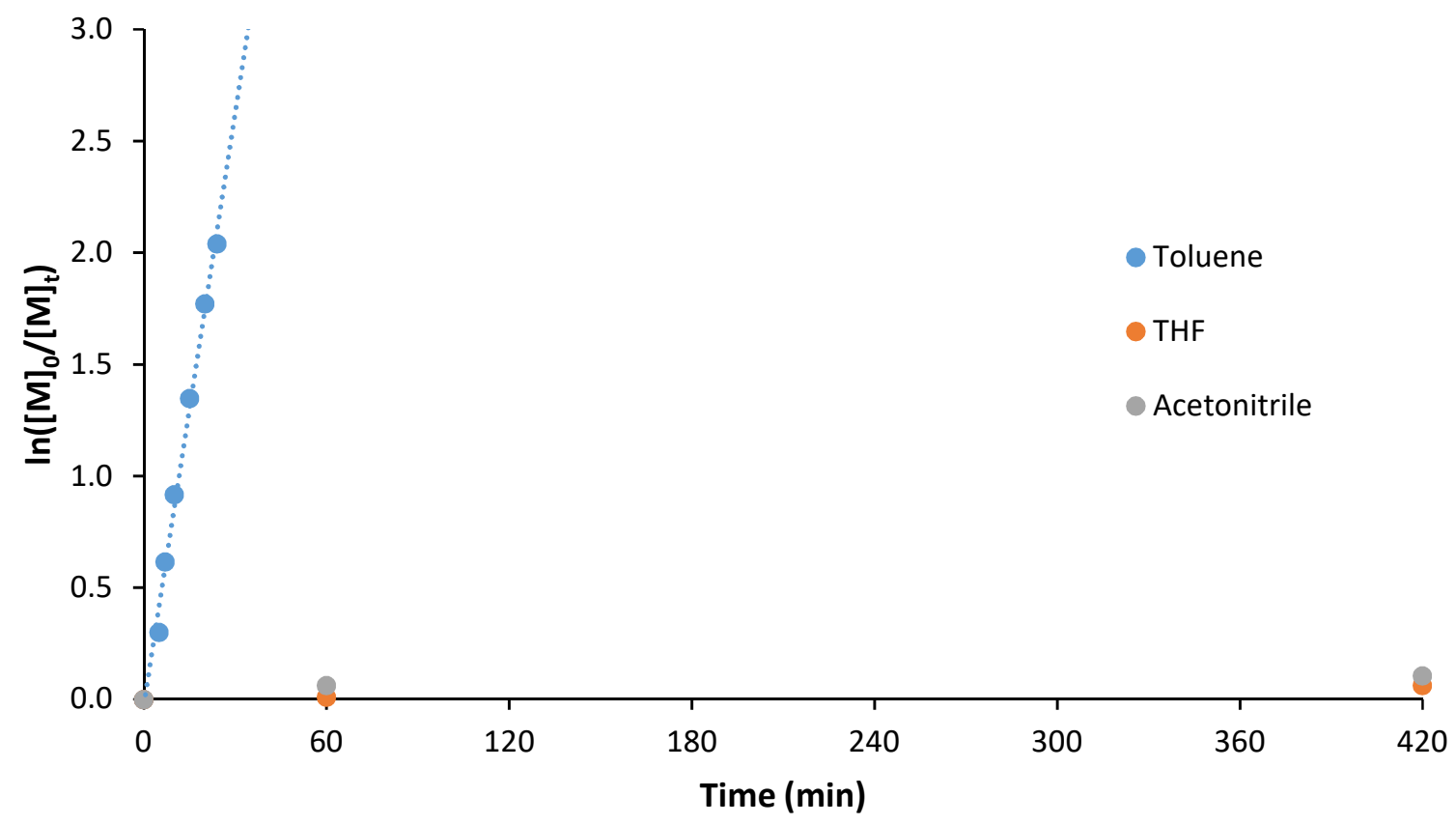

Conditions: $[\mathrm{MMA}]:[1]:[\mathrm{V}-70]=100: 1.0: 0.5, \mathrm{MMA}$ :solvent $=1: 1(\mathrm{v} / \mathrm{v}), 80^{\circ} \mathrm{C}$. Conversion determined by ${ }^{1} \mathrm{H}$ NMR spectroscopy.

Figure S3 MMA kinetics, with and without complex 1, using different concentrations of initiator

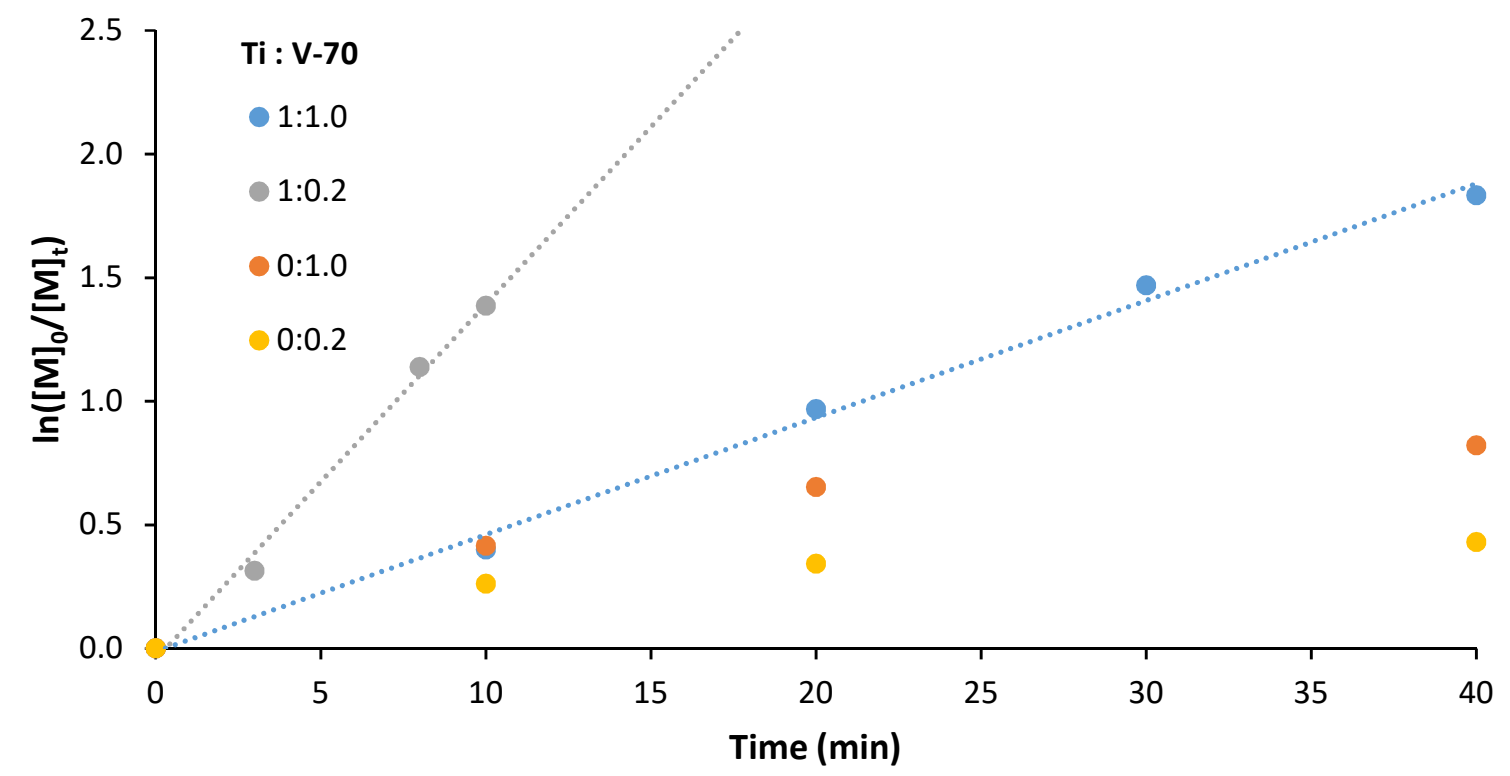

Conditions: $[\mathrm{MMA}]:[\mathbf{1}]:[\mathrm{V}-70]=100: \mathrm{x}: \mathrm{y}, \mathrm{MMA}:$ toluene $=1: 1(\mathrm{v} / \mathrm{v}), 80^{\circ} \mathrm{C}$. Conversion determined by ${ }^{1} \mathrm{H}$ NMR spectroscopy. 
Figure S4 MMA kinetics, with complex 1, using different concentrations of initiator
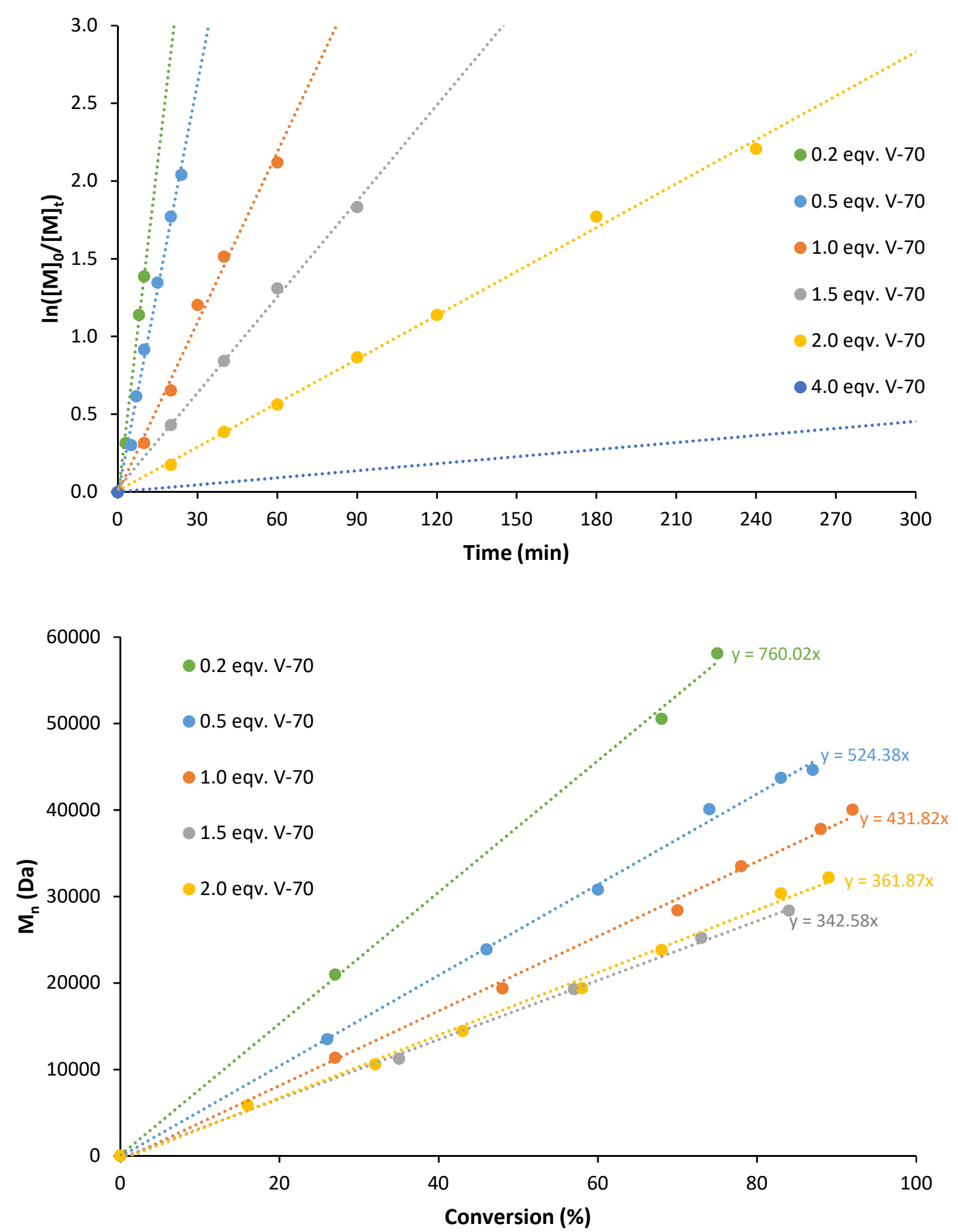

Conditions: $[\mathrm{MMA}]:[1]:[\mathrm{V}-70]=100: 1.00: \mathrm{x}, \mathrm{MMA}:$ toluene $=1: 1(\mathrm{v} / \mathrm{v}), 80^{\circ} \mathrm{C}$. Conversion determined by ${ }^{1} \mathrm{H}$ NMR spectroscopy. 
Figure S5 Addition of extra 1 during MMA polymerization

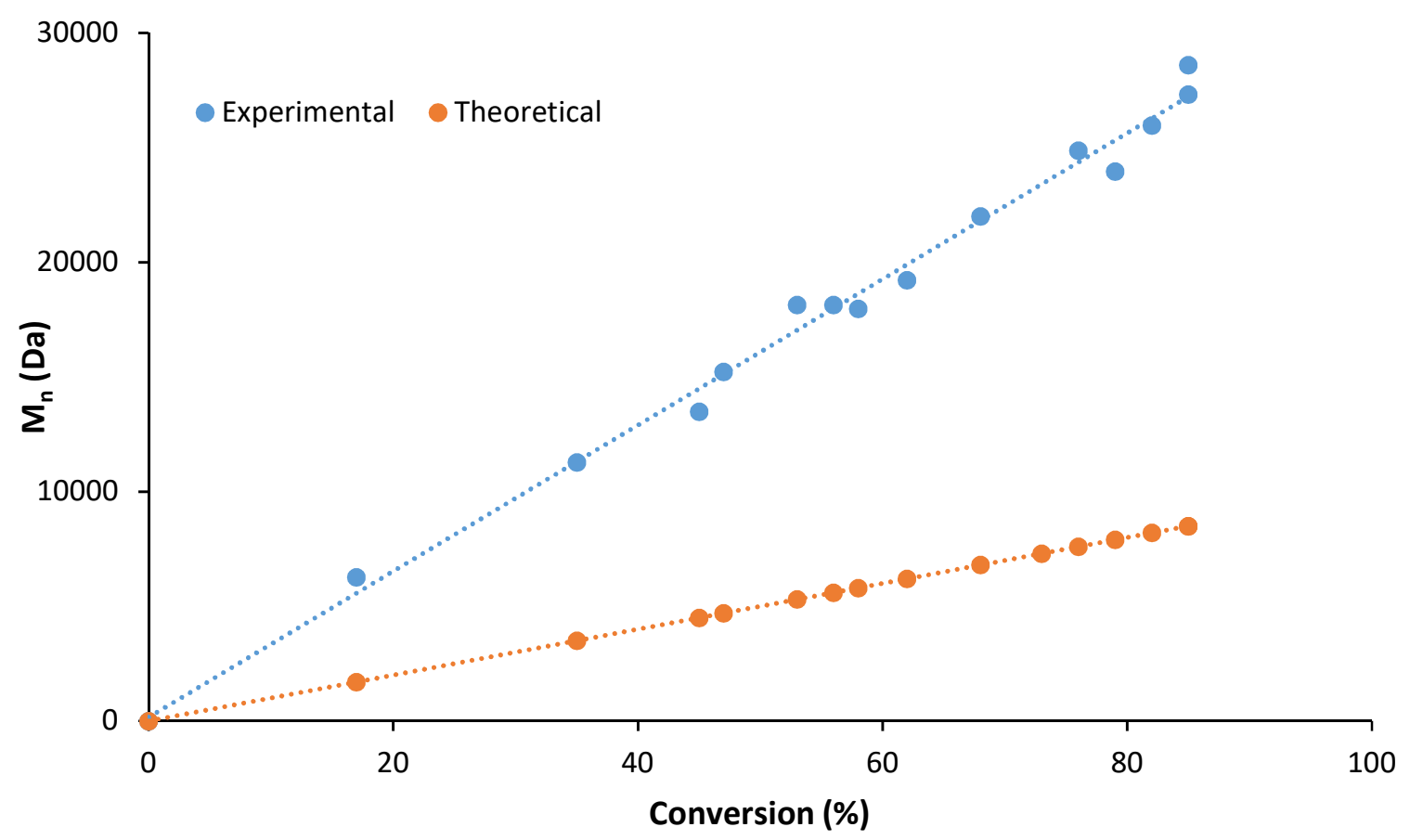

Conditions: [MMA]:[1]:[V-70] = 100:1.00:2.00, MMA:toluene $=1: 1(\mathrm{v} / \mathrm{v}), 80^{\circ} \mathrm{C}, 20 \mathrm{~min}$. Subsequently, add $\mathrm{x}$ eqv. 1, continue heating. Conversion determined by ${ }^{1} \mathrm{H}$ NMR spectroscopy. $\mathrm{M}_{n, t h}=[\mathrm{MMA}]_{0} /[\mathrm{Ti}(\mathrm{III})]_{0} \times \mathrm{M}(\mathrm{MMA}) \times$ conversion.

Figure S6 Use of a macroinitiator, with and without added 1

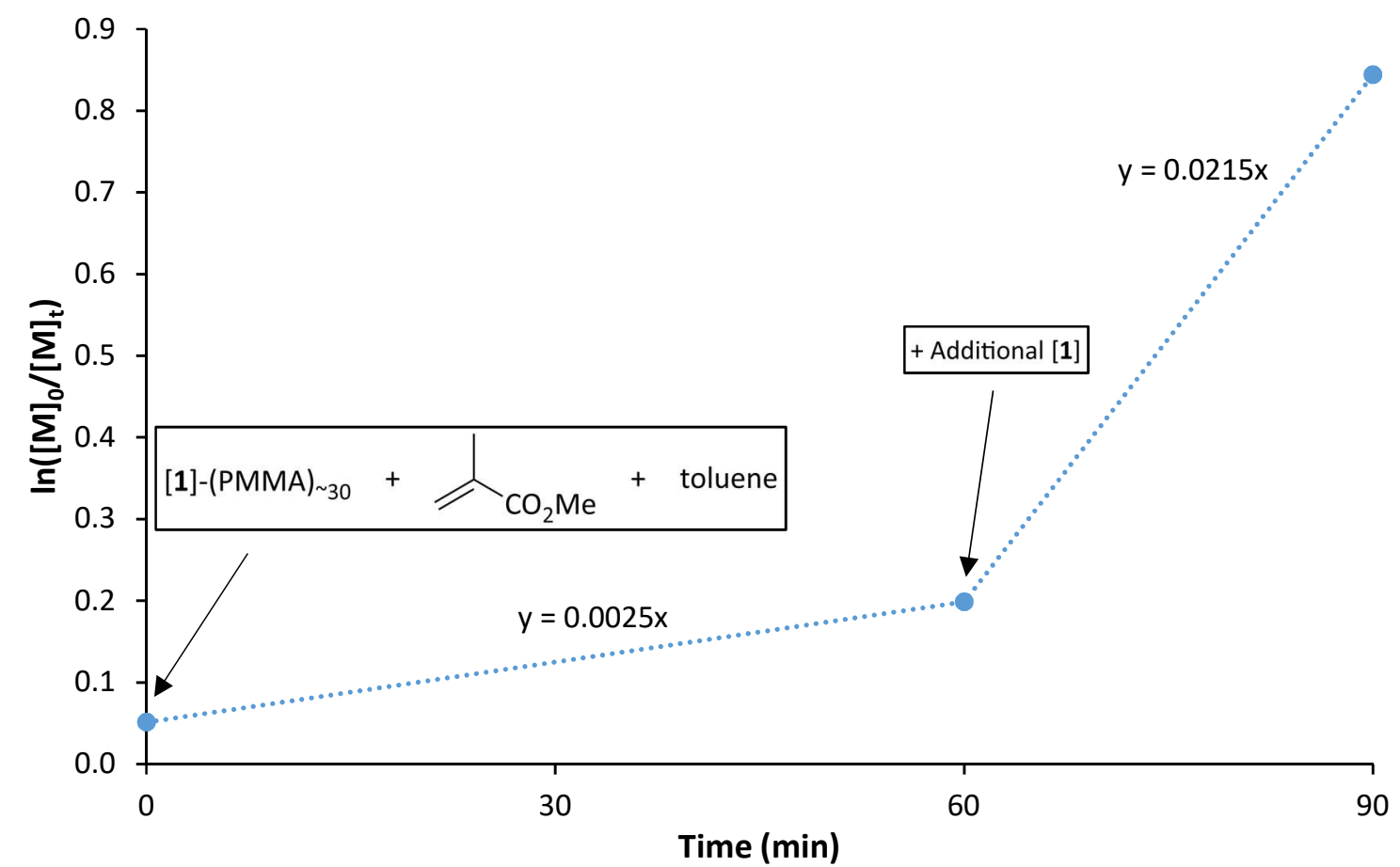

Conditions: MMA:toluene $=1: 1(\mathrm{v} / \mathrm{v}), 80^{\circ} \mathrm{C}$. Conversion determined by ${ }^{1} \mathrm{H}$ NMR spectroscopy. 
Figure $\mathbf{S 7}{ }^{\mathrm{n}}$ BuMA kinetics with complex 1
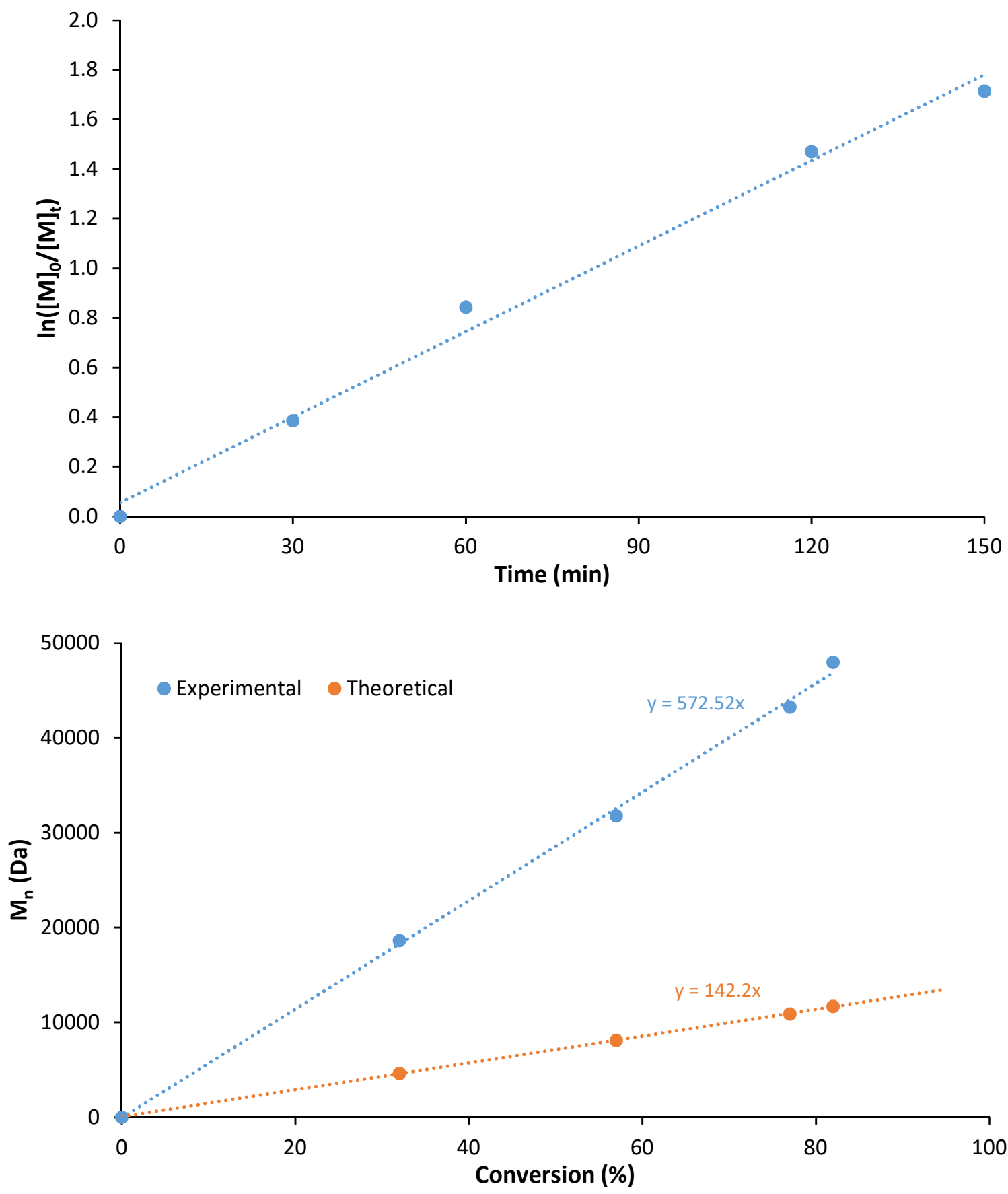

Conditions: [ $\left.{ }^{\mathrm{B} B M M}\right]:[1]:[\mathrm{V}-70]=100: 1.0: 1.0,{ }^{\mathrm{n} B u M A}$ :toluene $=1: 1(\mathrm{v} / \mathrm{v}) .80^{\circ} \mathrm{C}$. Conversion determined by ${ }^{1} \mathrm{H}$ NMR spectroscopy. $M_{n, t h}=\left[{ }^{n} B u M A\right]_{0} /[T i(I I I)]_{0} \times M\left({ }^{n} B u M A\right) \times$ conversion. 
Figure S8 MMA kinetics using different concentrations of complex 1
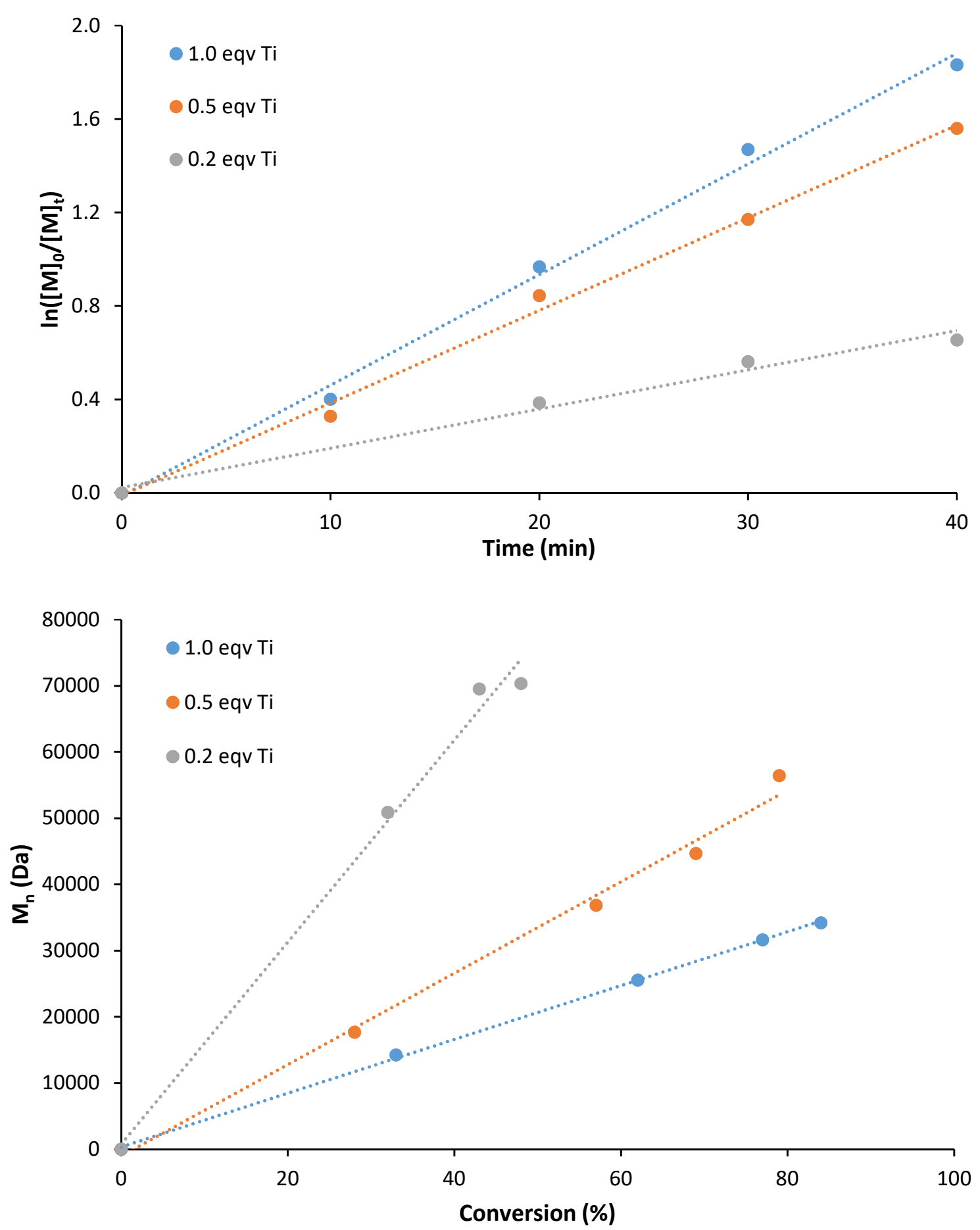

Conditions: $[\mathrm{MMA}]:[1]=100: \mathrm{x},[1]:[\mathrm{V}-70]=1.0: 1.0, \mathrm{MMA}:$ toluene $=1: 1(\mathrm{v} / \mathrm{v}) .80^{\circ} \mathrm{C}$. Conversion determined by ${ }^{1} \mathrm{H}$ NMR spectroscopy. 
Figure S9 MMA kinetics with complex 1 and 4
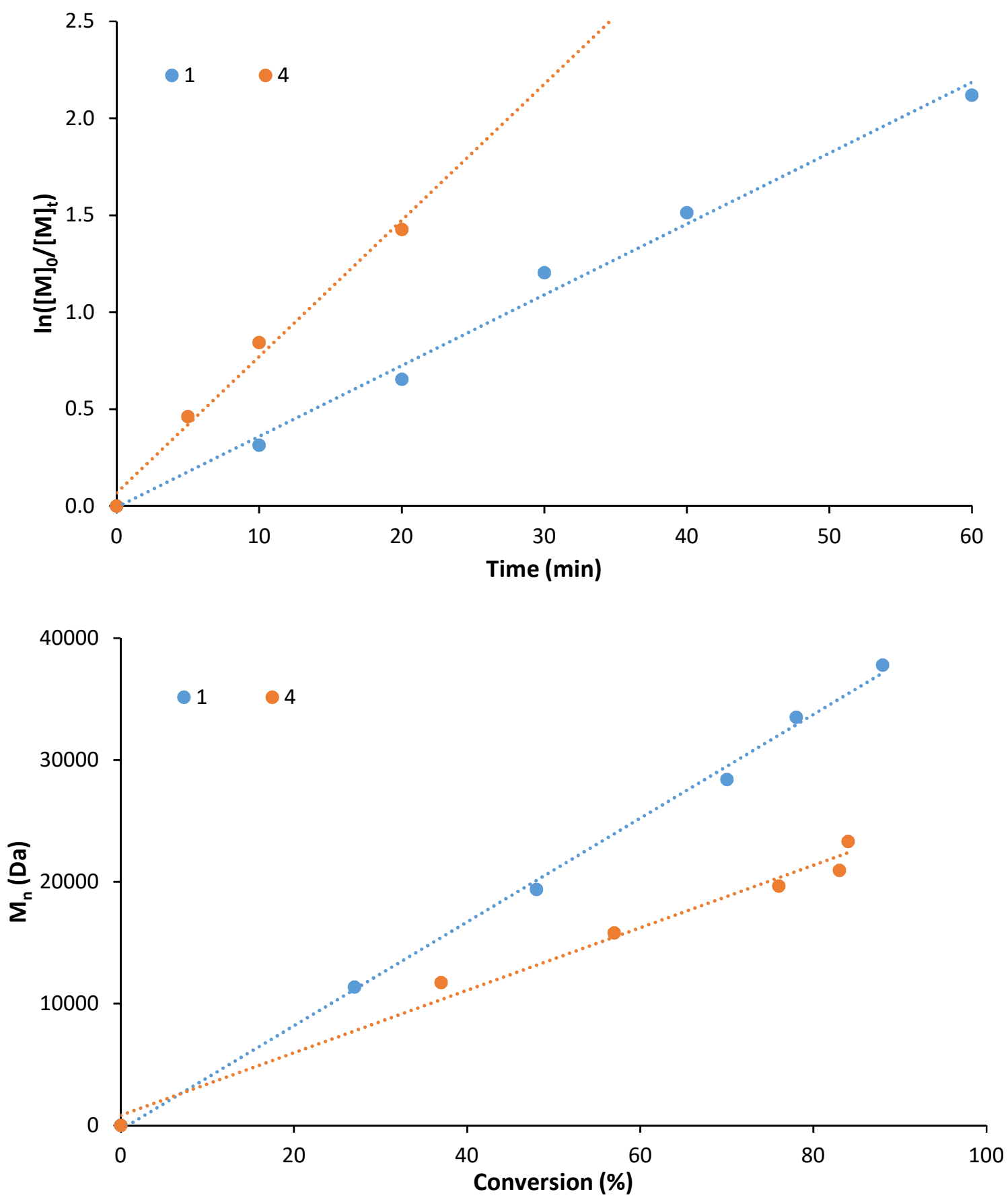

Conditions: $[\mathrm{MMA}]:[\mathrm{Ti}(\mathrm{III})]:[\mathrm{V}-70]=100: 1.0: 1.0, \mathrm{MMA}$ :toluene $=1: 1(\mathrm{v} / \mathrm{v}), 80^{\circ} \mathrm{C}$. Conversion determined by ${ }^{1} \mathrm{H}$ NMR spectroscopy. 


\section{MECHANISTIC PATHWAYS}

Scheme S3 Energy diagram showing the relative stability of 6-coordinate titanium species, relative to the 5coordinate complex

$\Delta \mathrm{G}_{1 \mathrm{M}, 298 \mathrm{~K}}(\mathrm{kcal} / \mathrm{mol})$

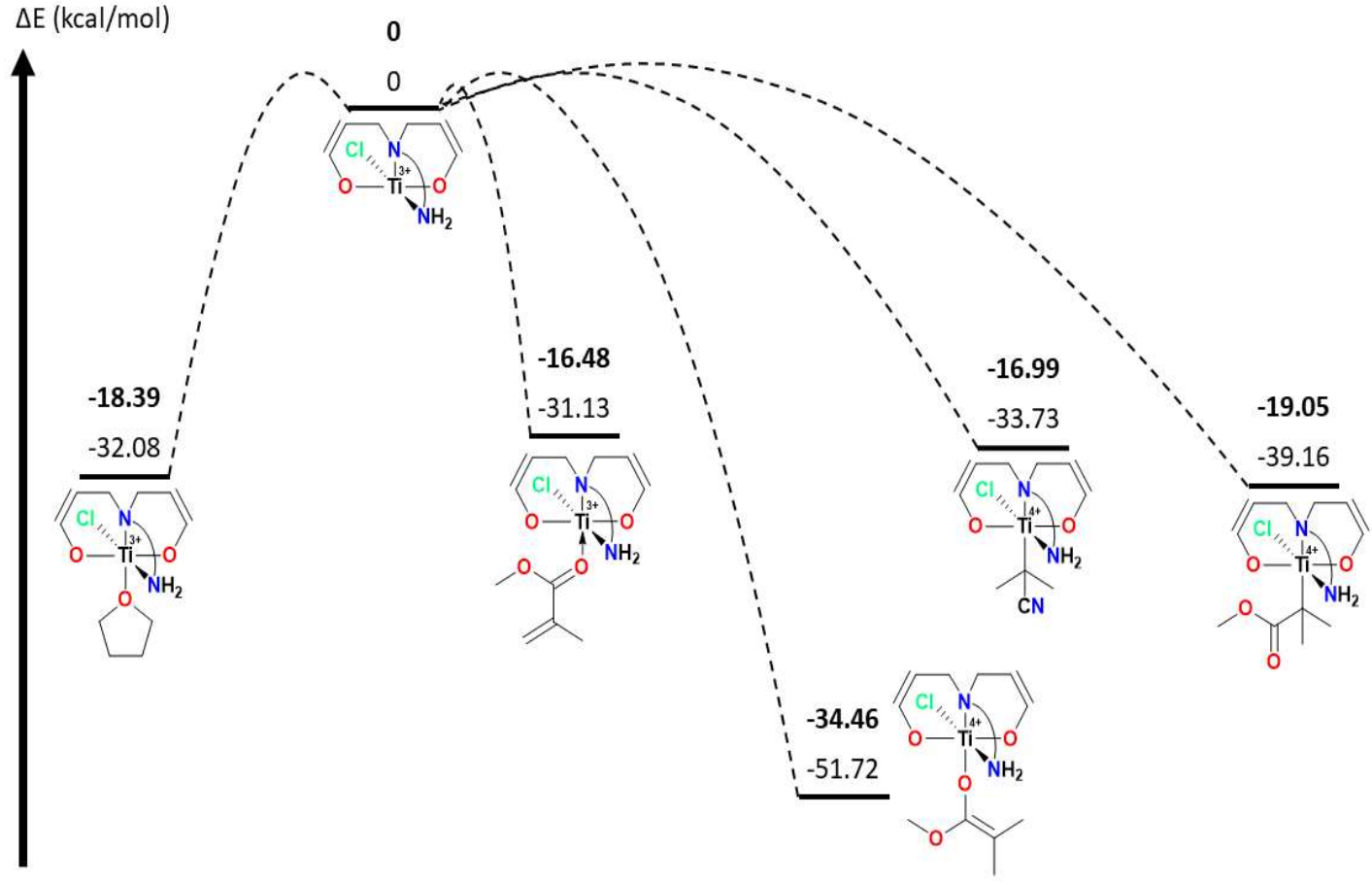

Scheme S4 Energy diagram showing the proposed bimetallic group transfer polymerization mechanism

\section{$\Delta \mathrm{G}_{1 \mathrm{M}, 298 \mathrm{~K}}(\mathrm{kcal} / \mathrm{mol})$ \\ $\Delta \mathrm{E}(\mathrm{kcal} / \mathrm{mol})$}

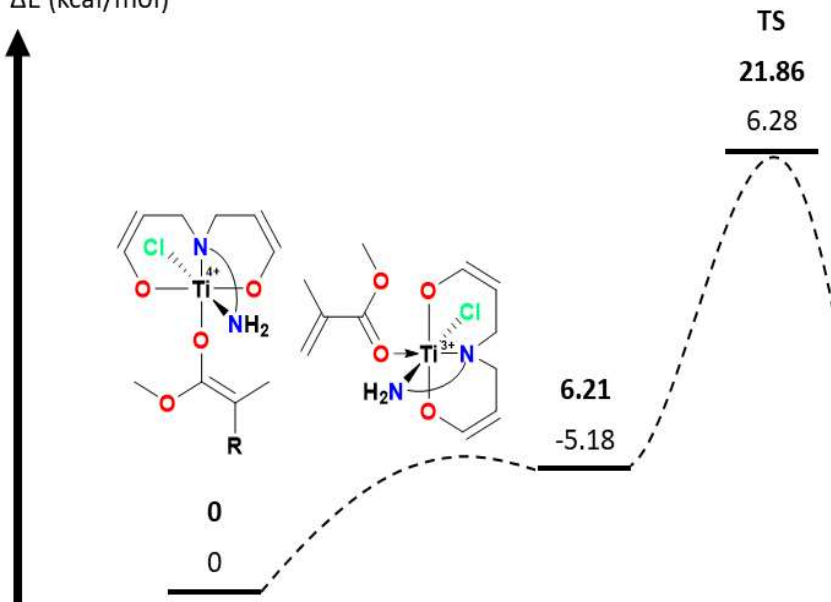

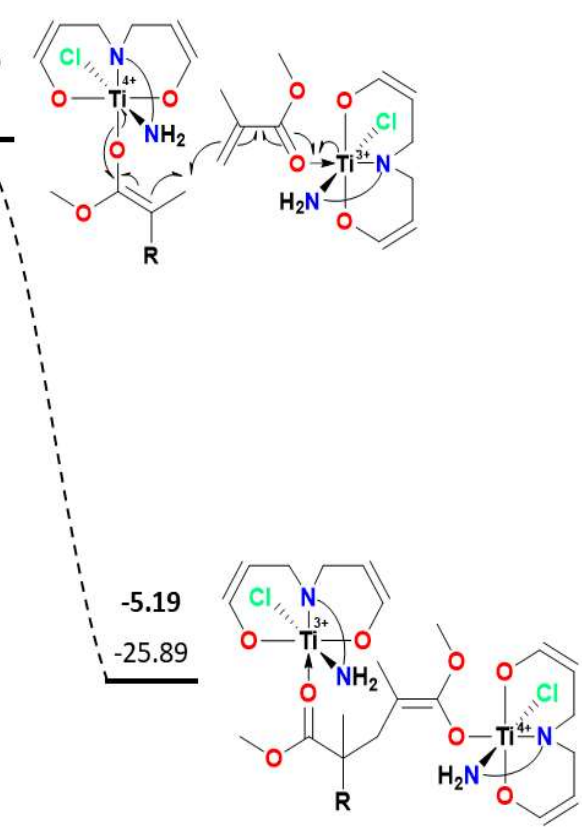


Scheme S5 Energy diagram showing the proposed monometallic group transfer polymerization mechanism

$\Delta \mathrm{G}_{1 \mathrm{M}, 298 \mathrm{~K}}(\mathrm{kcal} / \mathrm{mol})$

$\Delta \mathrm{E}(\mathrm{kcal} / \mathrm{mol})$

1
20.46

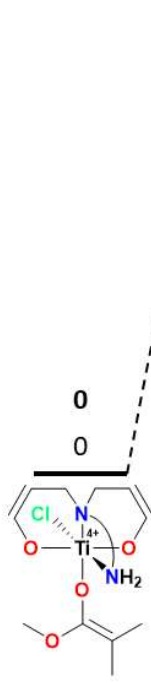

TS $\mathrm{H}_{\mathrm{N}^{-}} \mathrm{H}^{-\mathrm{H}}$

24.61

9.51 O II-

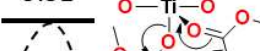

15.17

$\underbrace{1.77}_{\mathrm{H}_{-N}-\mathrm{H}^{-}}$,

$0-\mathrm{Ti}^{\mathrm{i}} \mathrm{O}$

少

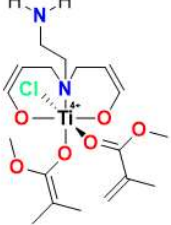




\section{MOLECULAR STRUCTURES}

Figure S10 Molecular structures of $\mathbf{2}$ (top), $\mathbf{3}$ (middle) and $\mathbf{4}$ (bottom) with ellipsoids set at the $50 \%$ probability level. Hydrogen atoms, solvent and minor disorder components have been omitted for clarity. Selected bond lengths $(\AA ̊ \AA)$ and angles $\left({ }^{\circ}\right)$ : 2 - Ti-Cl 2.4188(5), Ti-O1 1.8984(13), Ti-O2 1.8995(13), Ti-O3 2.2085(14), Ti-N1 2.2769(15), Ti-N2 2.2581(16), Cl-Ti-N1 175.10(4), O1-Ti-O2 167.43(6), N2-Ti-O3 175.39(6). 3 - Ti-Cl5 2.4209(5), Ti-O1 1.9158(11), Ti-O2 1.9072(11), Ti-O3 2.1415(12), Ti-N1 2.2350(13), Ti-N2 2.3052(13), Cl5-Ti-N2 177.97(4), O1-Ti-O2 172.47(5), N1-Ti-O3 169.50(5). 4 - Ti-Cl 2.4185(6), Ti-O1 1.8893(16), Ti-O2 1.9211(16), Ti-O3 2.1142(17), Ti-O4 2.1856(17), Ti-N 2.2847(17), Cl-Ti-N 170.48(5), O1-Ti-O2 166.07(6), O3-Ti-O4 174.28(6).
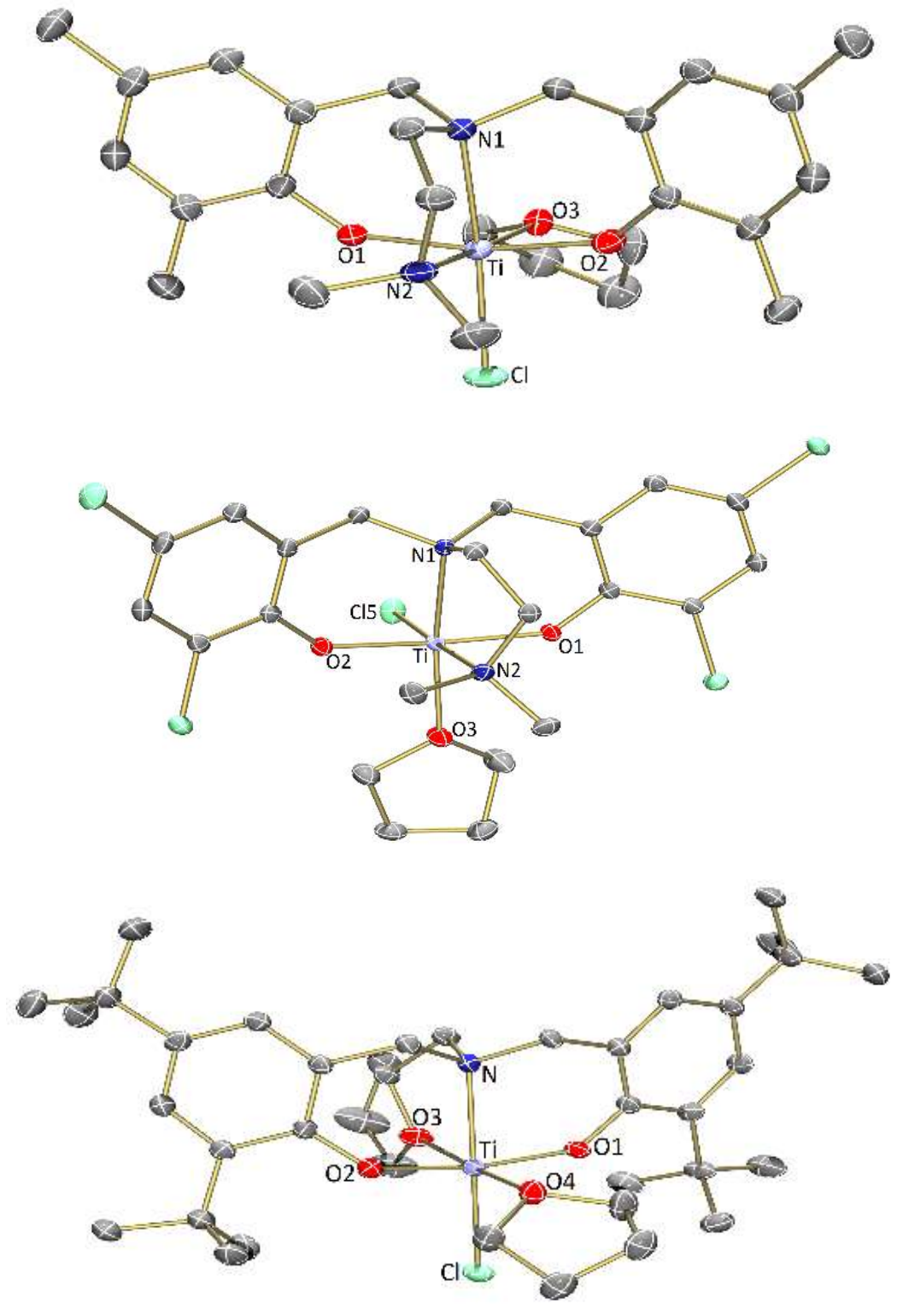
Figure S11 Molecular structures of $\mathbf{2}(\boldsymbol{\mu}-\mathrm{Cl})_{2}$ (top) and $\mathbf{3}(\boldsymbol{\mu}-\mathrm{Cl})_{2}$ (bottom) with ellipsoids set at the $50 \%$ probability level. Hydrogen atoms and solvent have been omitted for clarity. Selected bond lengths $(\AA)$ and angles $\left({ }^{\circ}\right): \mathbf{2}(\boldsymbol{\mu}-$ $\mathrm{Cl}_{2}$ - Ti---Ti 3.2506(7), Ti-Cl 2.4568(6), Ti-Cl' 2.4847(6), Ti-O1 1.8875(16), Ti-O2 1.8734(15), Ti-N1 2.2827(18), TiN2 2.2891(19), Cl-Ti-N1 169.13(5), Cl'-Ti-N2 171.80(5), O1-Ti-O2 167.81(7). 3( $\mu$-Cl)2 - Ti---Ti 3.2002(8), Ti-Cl5 2.4463(6), Ti-Cl5' 2.4635(7), Ti-O1 1.8880(16), Ti-O2 1.8946(16), Ti-N1 2.2776(18), Ti-N2 2.275(2), Cl5-Ti-N1 168.47(6), Cl5'-Ti-N2 171.98(6), O1-Ti-O2 168.42(7).

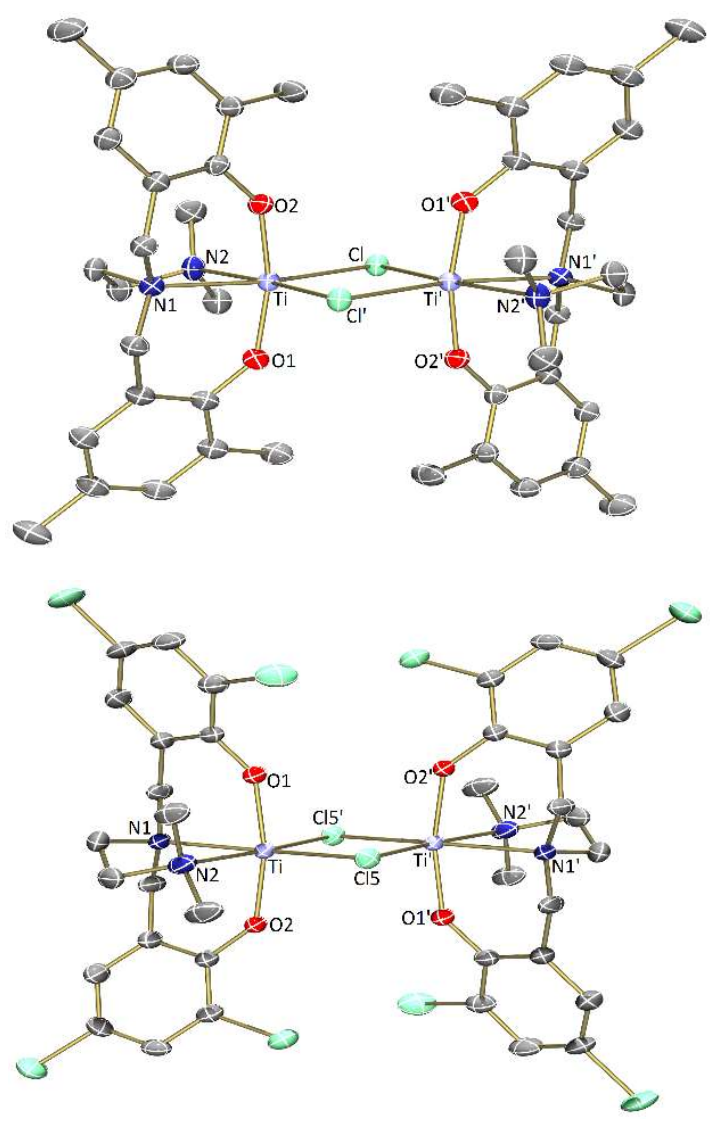

Figure S12 Dimeric structure of complex 7 (top) and views along the two different $\mathrm{N}$-Ti-Obridge axes showing pseudo- $\Delta$ (bottom left) and pseudo- $\Lambda$ (bottom right) configurations

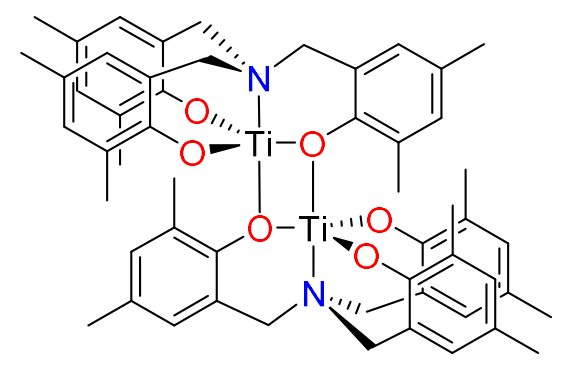

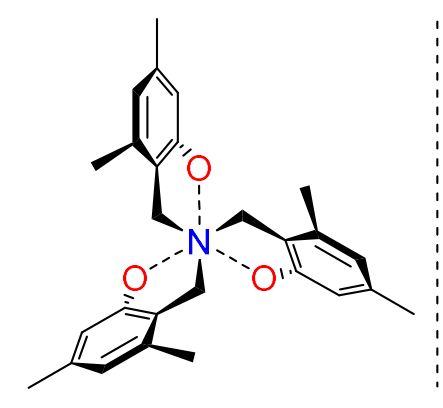

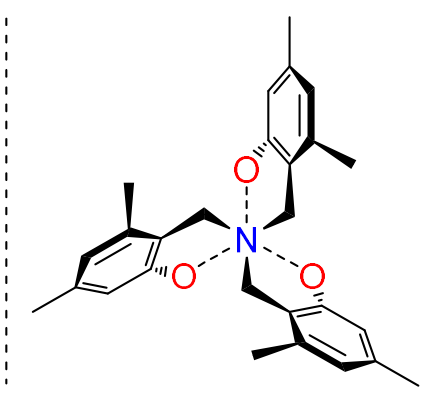


NMR SPECTRA

Figure $\mathbf{S 1 3}{ }^{1} \mathrm{H}$ NMR spectrum of complex $2(\boldsymbol{\mu}-\mathrm{Cl})_{2}\left(500 \mathrm{MHz}, \mathrm{C}_{6} \mathrm{D}_{6}\right)$

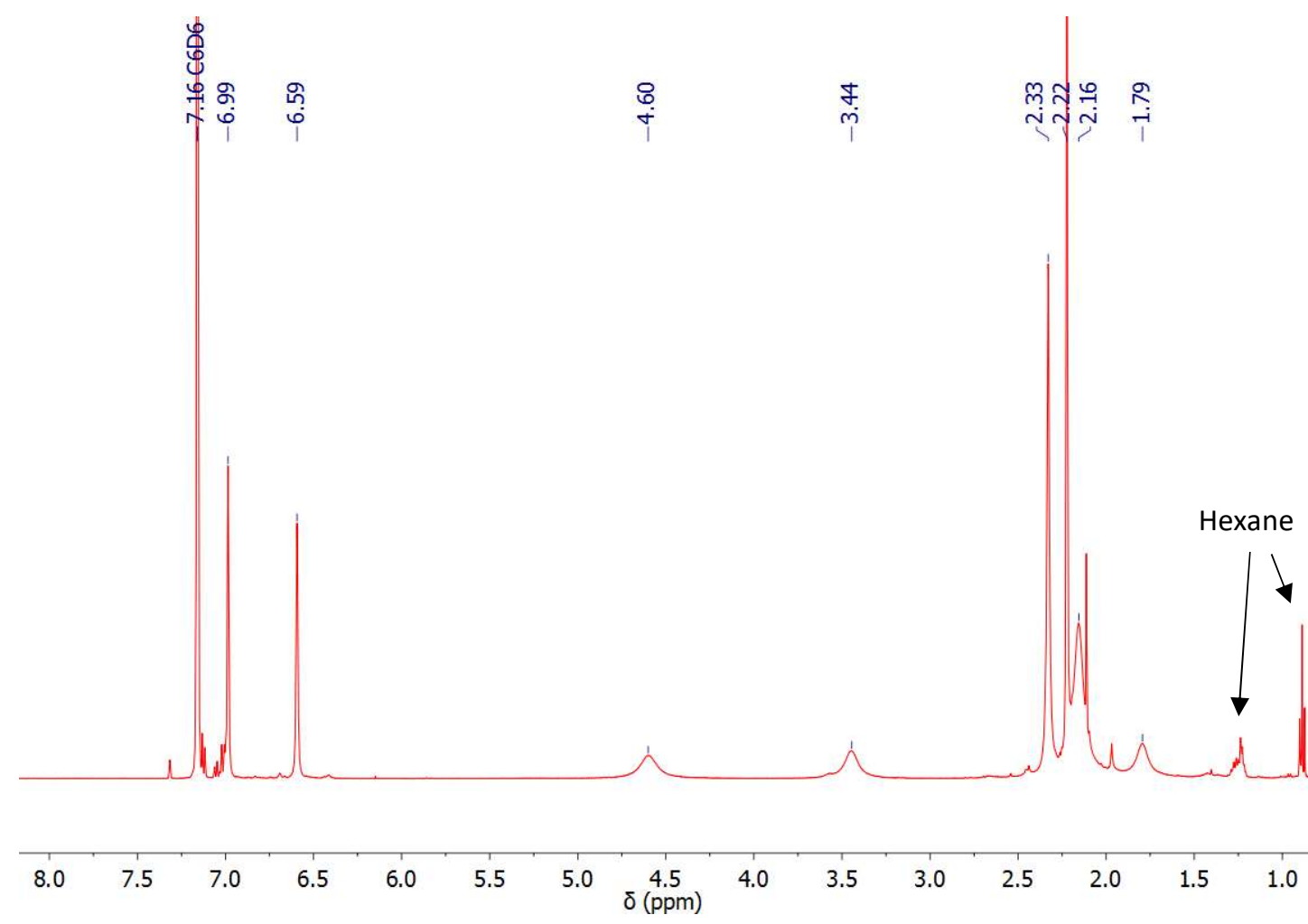

Figure S14 ${ }^{1} \mathrm{H}$ NMR spectrum of complex $\mathbf{3}(\boldsymbol{\mu}-\mathrm{Cl})_{2}\left(500 \mathrm{MHz}, \mathrm{C}_{6} \mathrm{D}_{6}\right)$

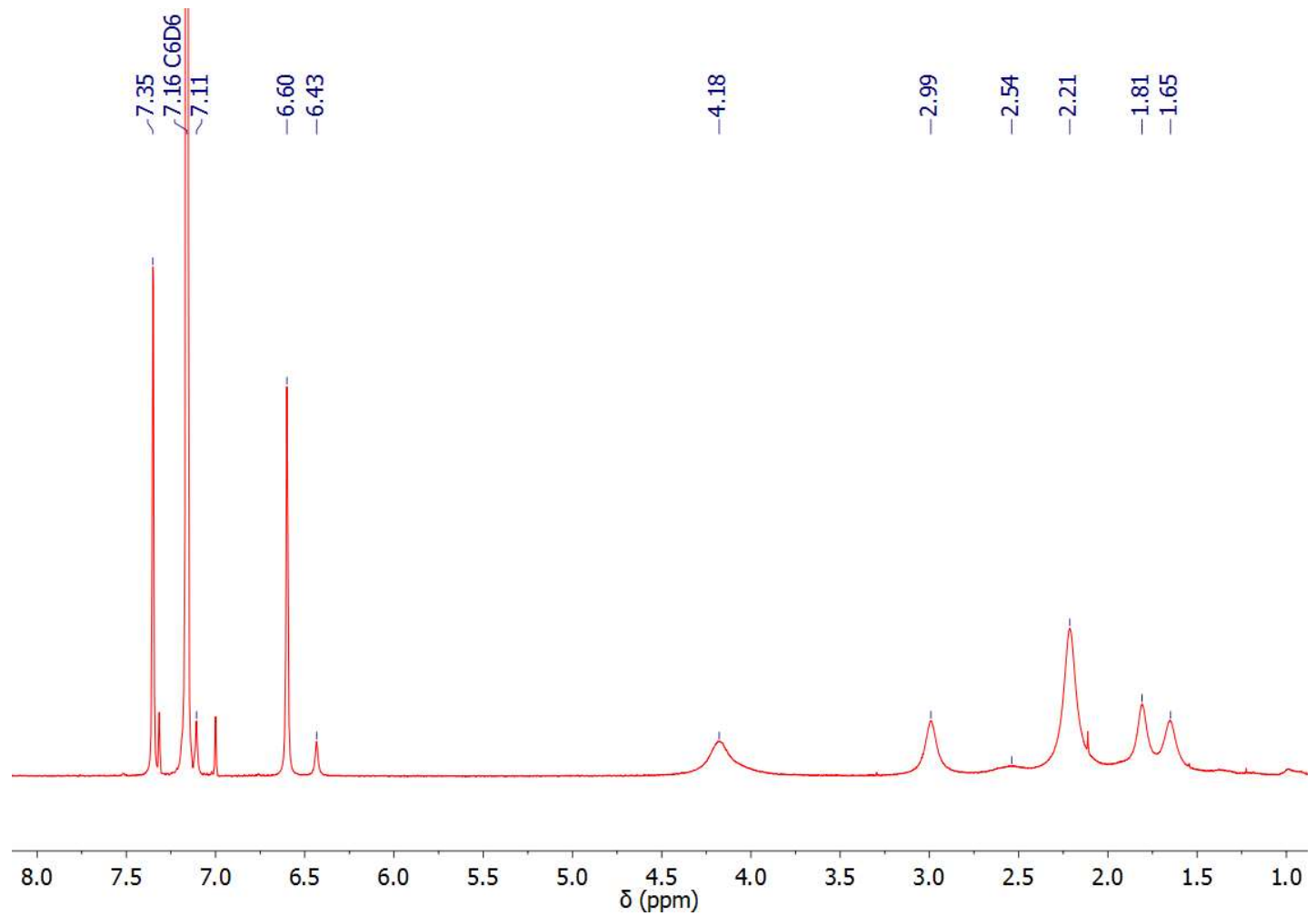


Figure $\mathrm{S} 15{ }^{1} \mathrm{H}$ NMR spectrum of complex $6\left(500 \mathrm{MHz}, \mathrm{C}_{6} \mathrm{D}_{6}\right)$
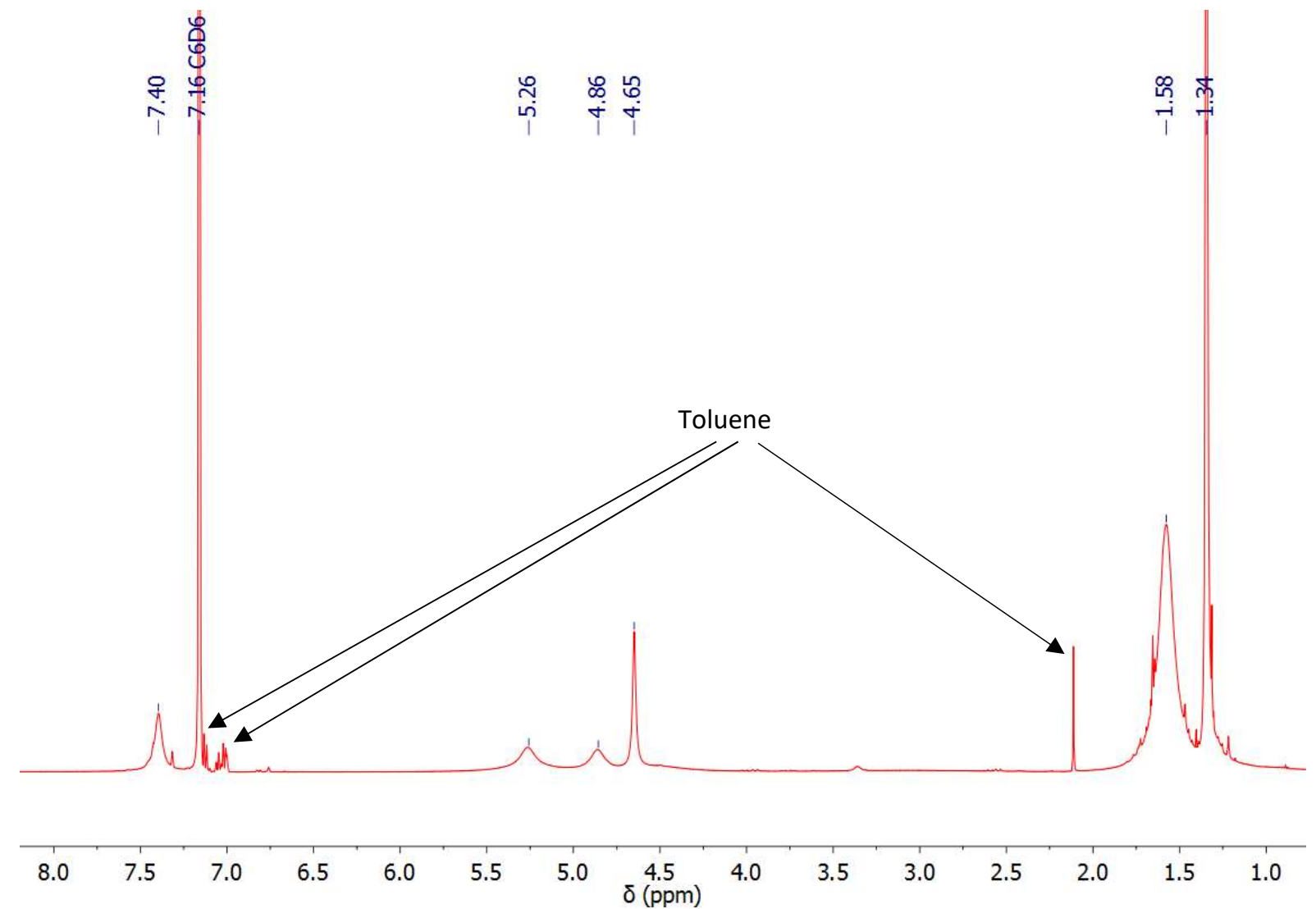

Figure $\mathrm{S} 16{ }^{1} \mathrm{H}$ NMR spectrum of complex $7\left(500 \mathrm{MHz}, \mathrm{C}_{6} \mathrm{D}_{6}\right)$

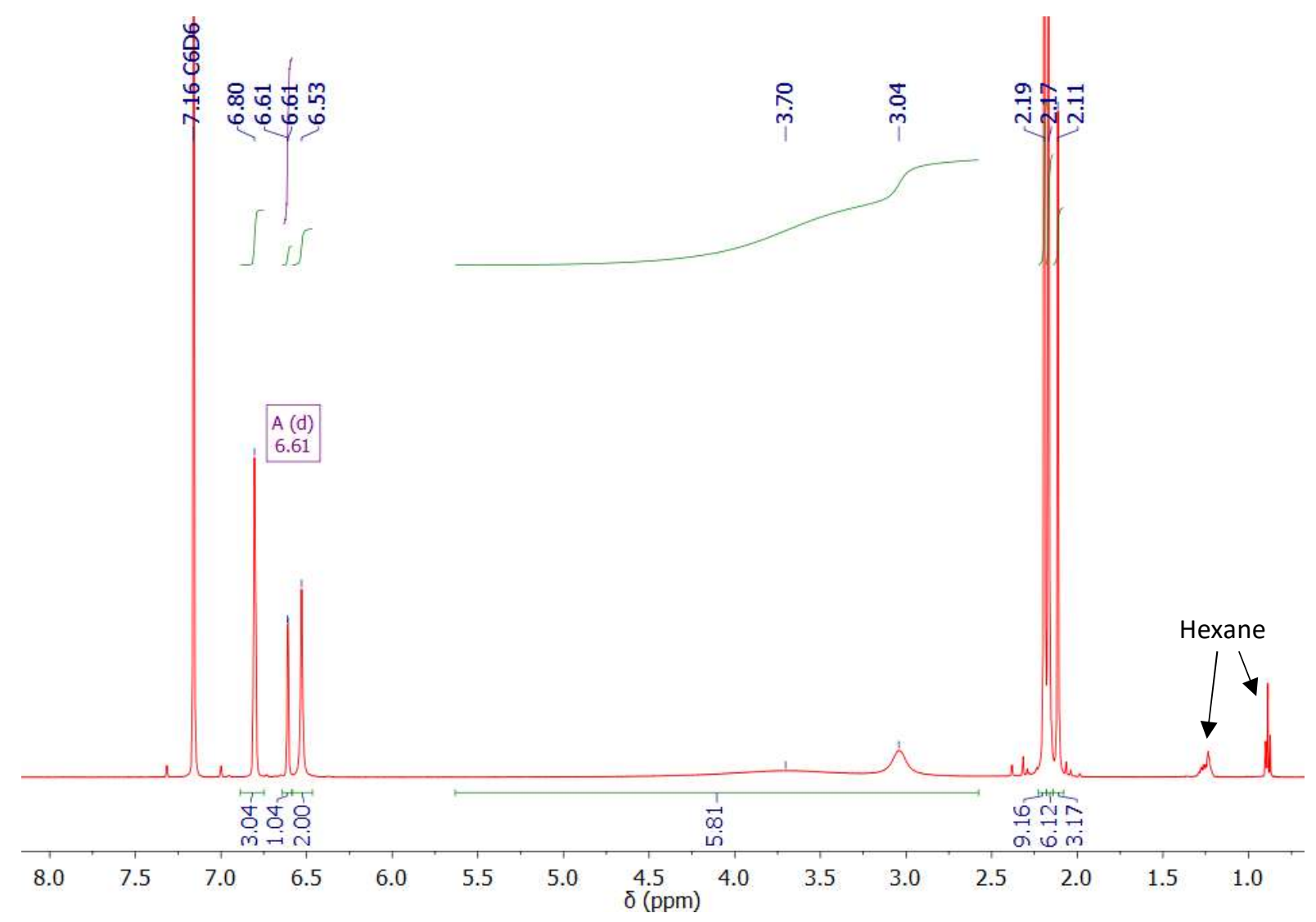


Figure $\mathrm{S} 17{ }^{13} \mathrm{C}\left\{{ }^{1} \mathrm{H}\right\}$ NMR spectrum of complex $7\left(126 \mathrm{MHz}, \mathrm{C}_{6} \mathrm{D}_{6}\right)$

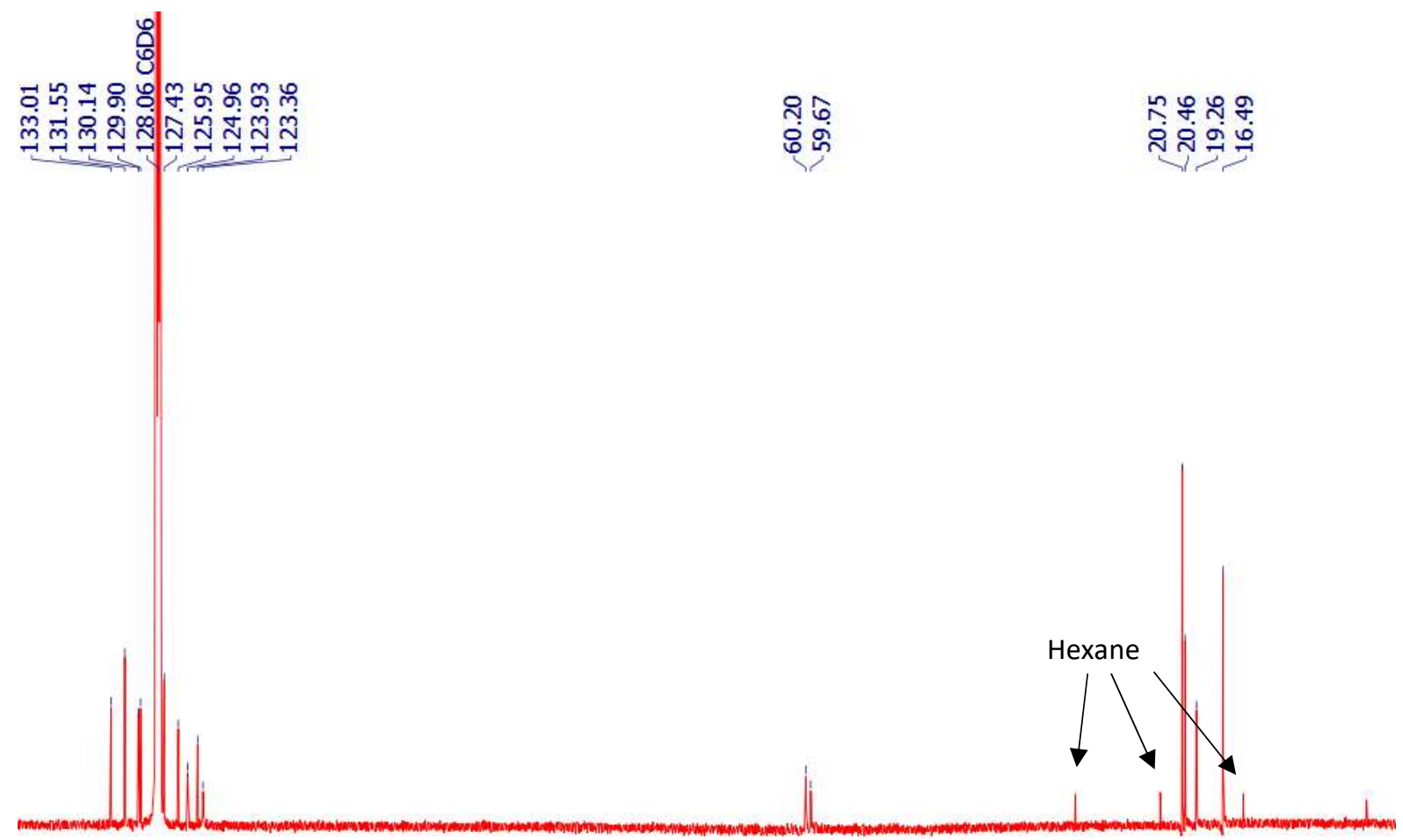

$14013513012512011511010510095 \quad 90 \quad 85 \quad 80 \begin{gathered}75 \\ \delta(\mathrm{ppm})\end{gathered}$

Figure S18 Variable temperature (203-363 K (top-bottom)) ${ }^{1} \mathrm{H}$ NMR spectra of complex 7 (400 MHz, $\left.\mathrm{C}_{6} \mathrm{D}_{6}\right)$

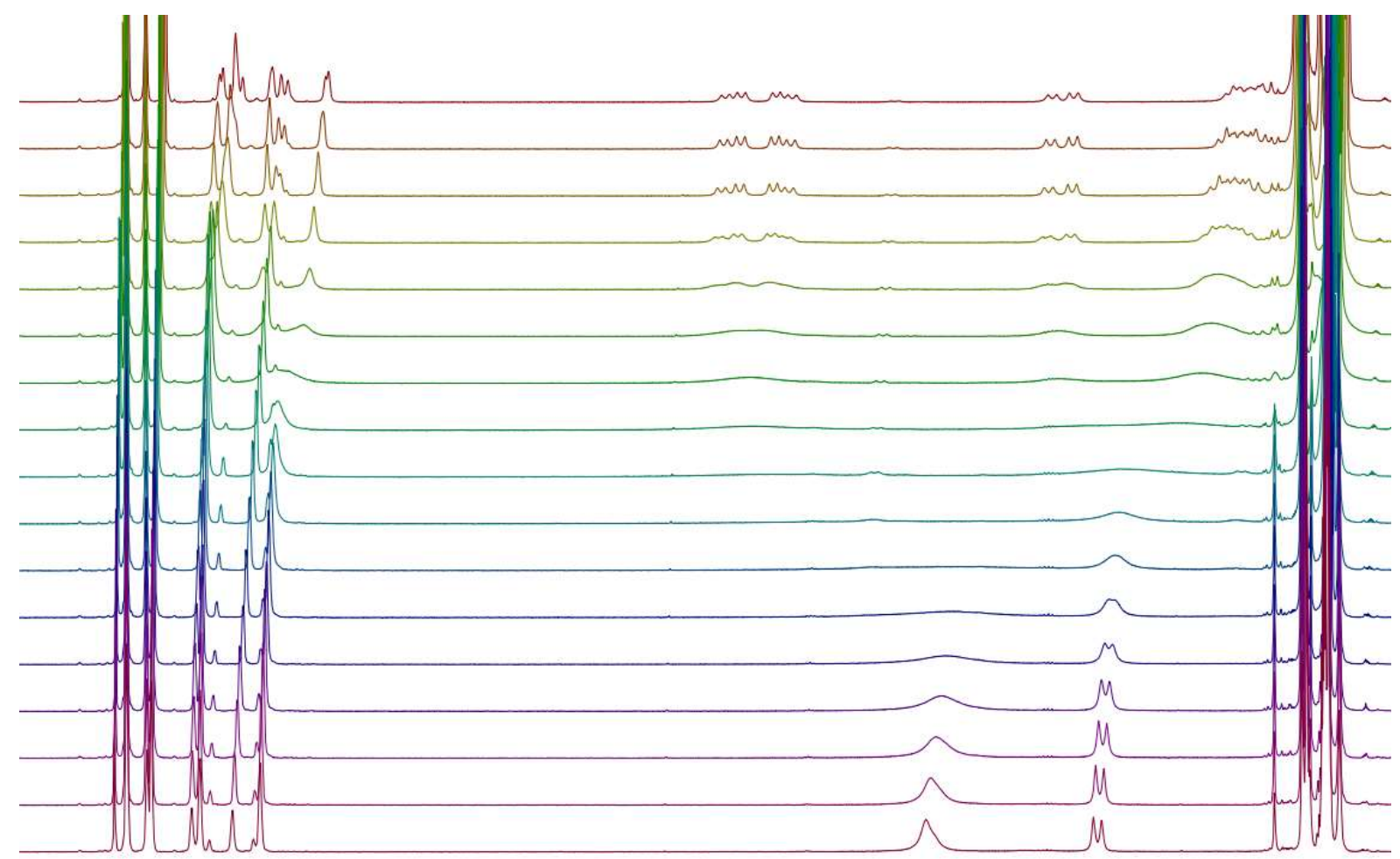

$\begin{array}{llllllllllllllllllllllllllll}7.4 & 7.2 & 7.0 & 6.8 & 6.6 & 6.4 & 6.2 & 6.0 & 5.8 & 5.6 & 5.4 & 5.2 & 5.0 & 4.8 & 4.6 & 4.4 & 4.2 & 4.0 & 3.8 & 3.6 & 3.4 & 3.2 & 3.0 & 2.8 & 2.6 & 2.4 & 2.2 & 2.0\end{array}$ $\delta(\mathrm{ppm})$ 
Figure S19 Variable temperature (203-363 K (top-bottom)) ${ }^{1} \mathrm{H}$ NMR spectra of complex $7(400 \mathrm{MHz}$, d8toluene)

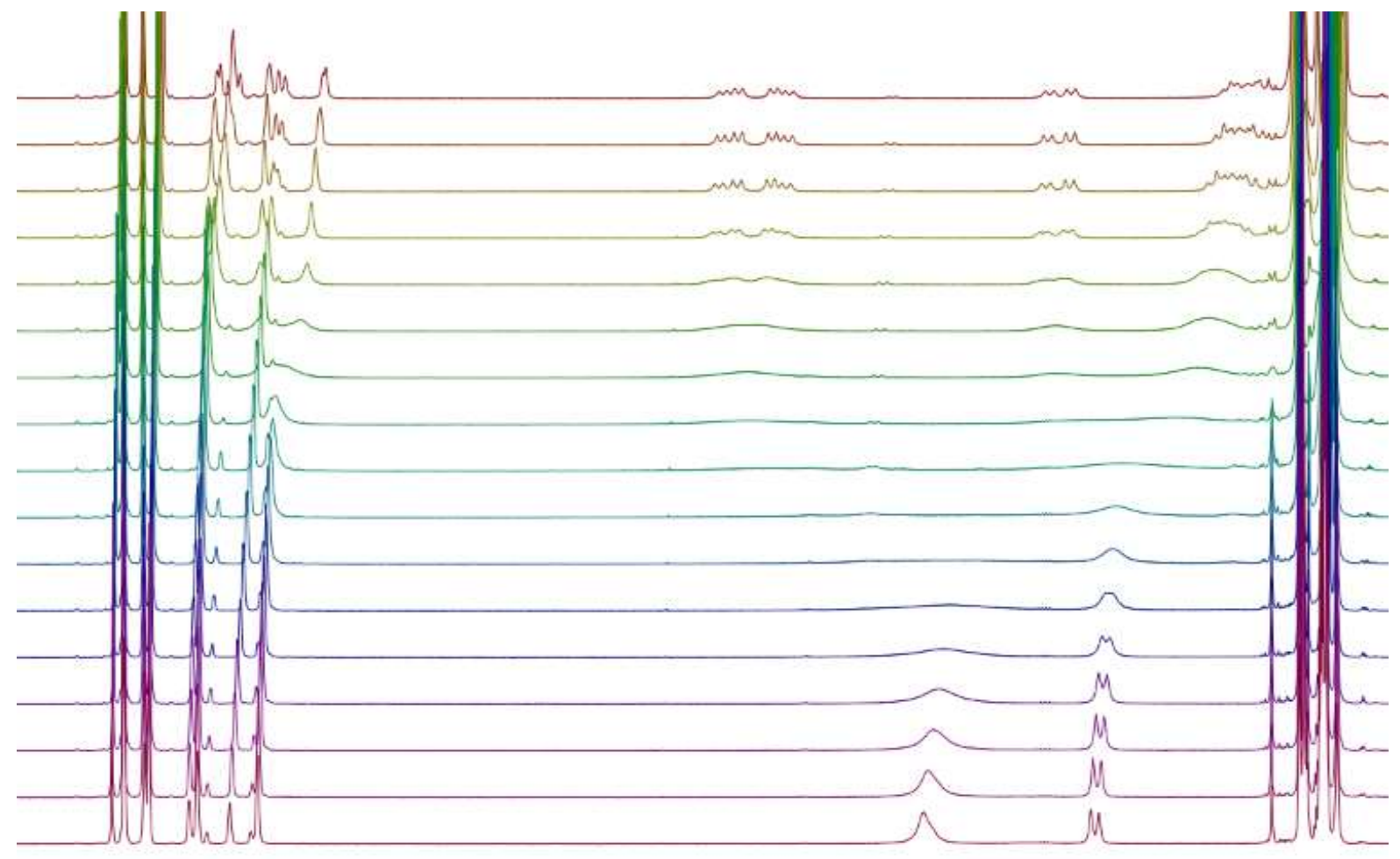

$\begin{array}{llllllllllllllllllllllllllllllll}7.4 & 7.2 & 7.0 & 6.8 & 6.6 & 6.4 & 6.2 & 6.0 & 5.8 & 5.6 & 5.4 & 5.2 & 5.0 & \underset{\delta}{\delta}(\mathrm{ppm}) & 4.8 & 4.6 & 4.4 & 4.2 & 4.0 & 3.8 & 3.6 & 3.4 & 3.2 & 3.0 & 2.8 & 2.6 & 2.4 & 2.2 & 2.0\end{array}$

Figure S20 Determination of the tacticity of purified poly(methyl methacrylate) by ${ }^{1} \mathrm{H}$ NMR spectroscopy $\left(\mathrm{CDCl}_{3}\right)$

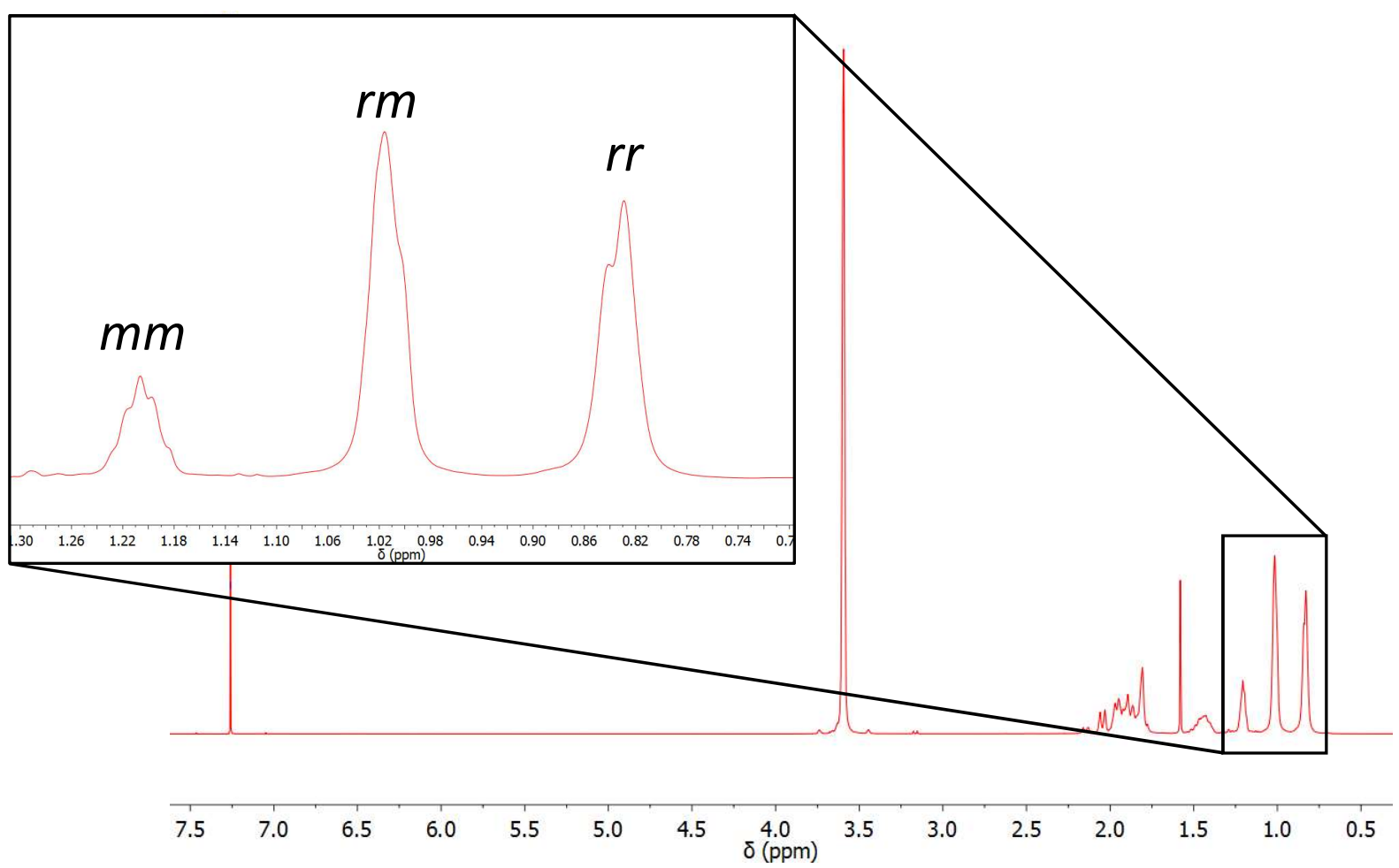


Figure S21 ${ }^{1} \mathrm{H}$ NMR spectrum of purified poly(methyl methacrylate) produced by complex $7\left(500 \mathrm{MHz}^{\mathrm{CDCl}} \mathrm{CD}_{3}\right)$

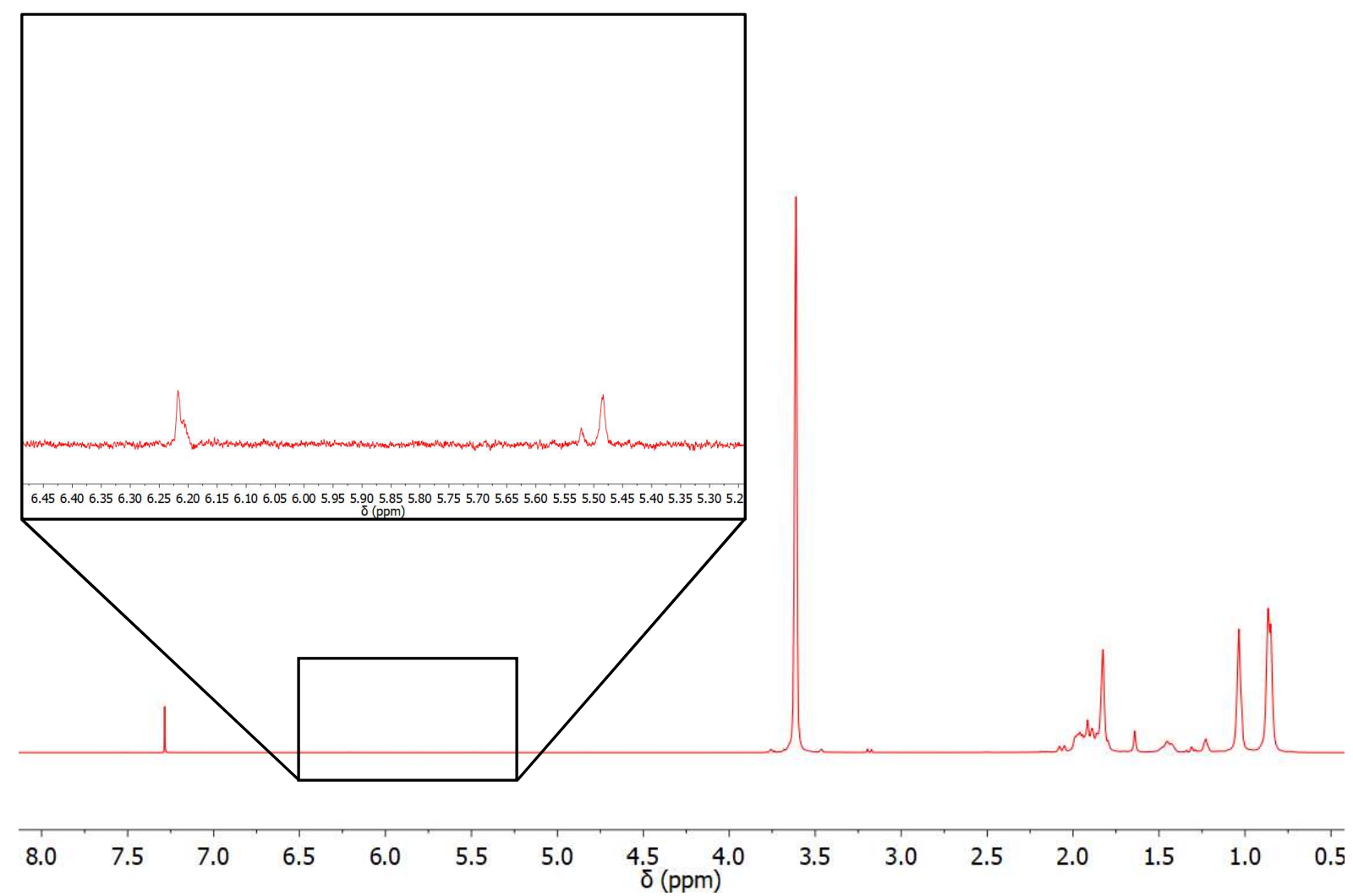

Figure S22 Overlaid ${ }^{1} \mathrm{H}$ NMR spectra of pure complex $\mathbf{1}$ (bottom) and product of reaction between complex $\mathbf{1}$ and $\mathrm{V}-70\left(80^{\circ} \mathrm{C}, 30 \mathrm{mins}\right)$ (top) $\left(600 \mathrm{MHz}, \mathrm{C}_{6} \mathrm{D}_{6}\right)$

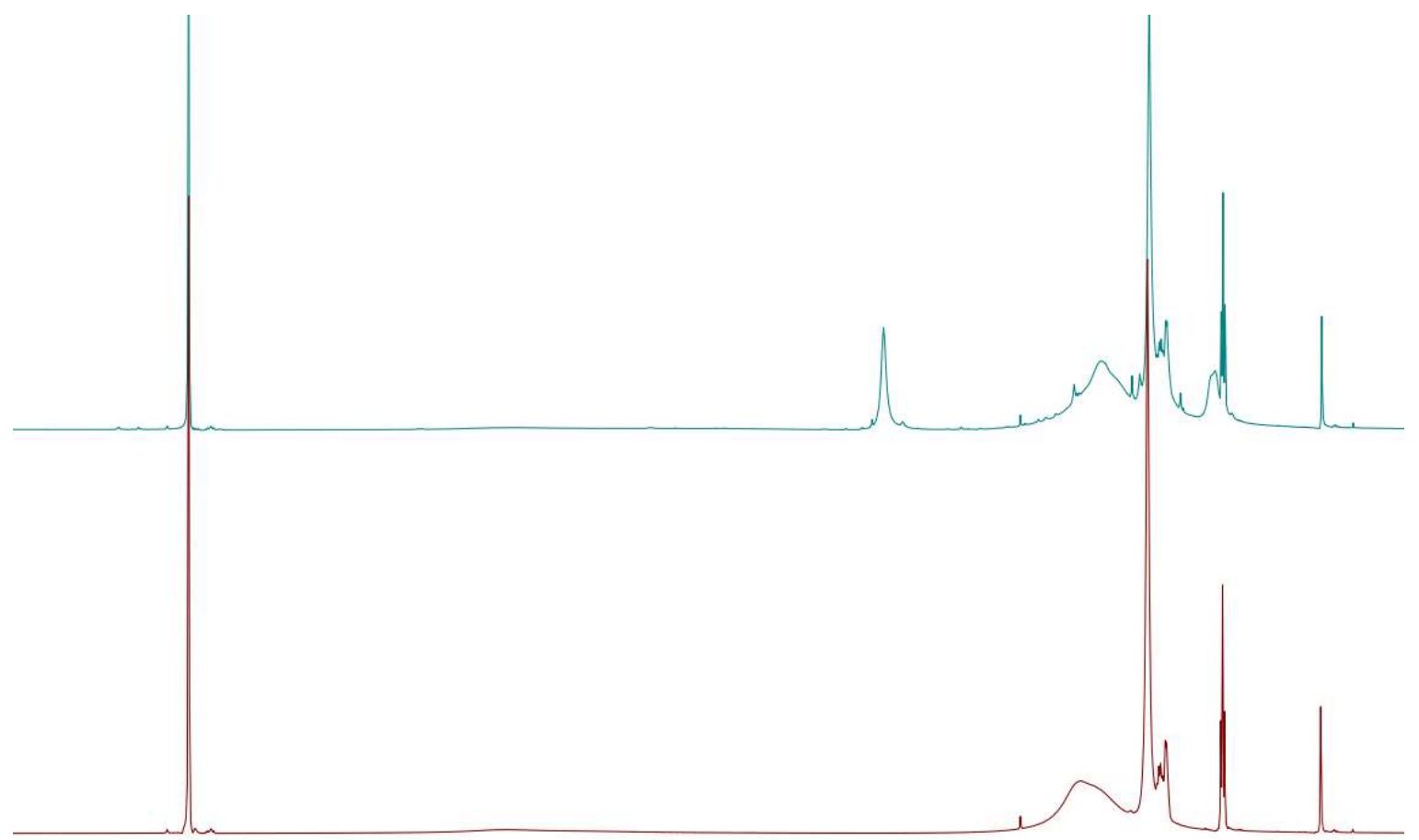

$\begin{array}{lllllllllllllllll}8.0 & 7.5 & 7.0 & 6.5 & 6.0 & 5.5 & 5.0 & 4.5 & \begin{array}{c}4.0 \\ \delta(\mathrm{ppm})\end{array} & 3.5 & 3.0 & 2.5 & 2.0 & 1.5 & 1.0 & 0.5 & 0.0\end{array}$


Figure S23 Overlaid ${ }^{1} \mathrm{H}$ NMR spectra of complex 1 and V-601 (bottom) and product of reaction between complex 1 and $\mathrm{V}$-601 ( $80^{\circ} \mathrm{C}, 20$ hours) (top) $\left(500 \mathrm{MHz}, \mathrm{C}_{6} \mathrm{D}_{6}\right)$

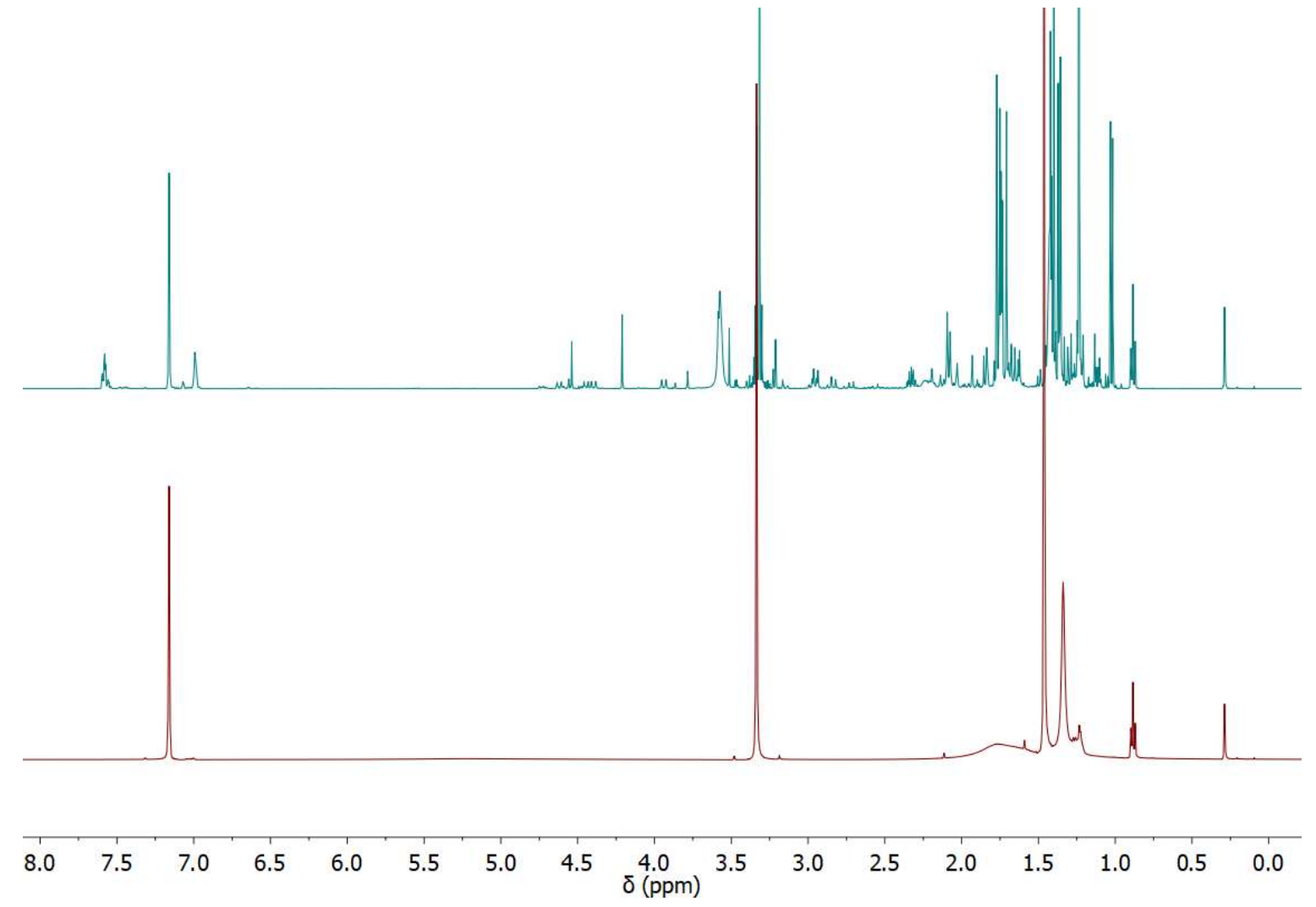




\section{X-RAY CRYSTALLOGRAPHIC DATA}

Figure S24 Molecular structure of $\mathbf{2}$ with ellipsoids set at the 50\% probability level. Hydrogen atoms and disordered solvent have been omitted for clarity.

Note. Residual electron density could be modelled as part of a molecule of THF. 'Growing' the THF reveals that the THF molecule is in fact disordered over two positions about an inversion centre. One atom of the THF (C27B) was found to be common to both components of the disorder, and hence was assigned an SOF of 1 . The other atoms (O4, C28, C29) were assigned SOFs of 0.5. A number of 'FREE' and 'BIND' commands were required to allow chemically sensible placement of $H$ atoms.

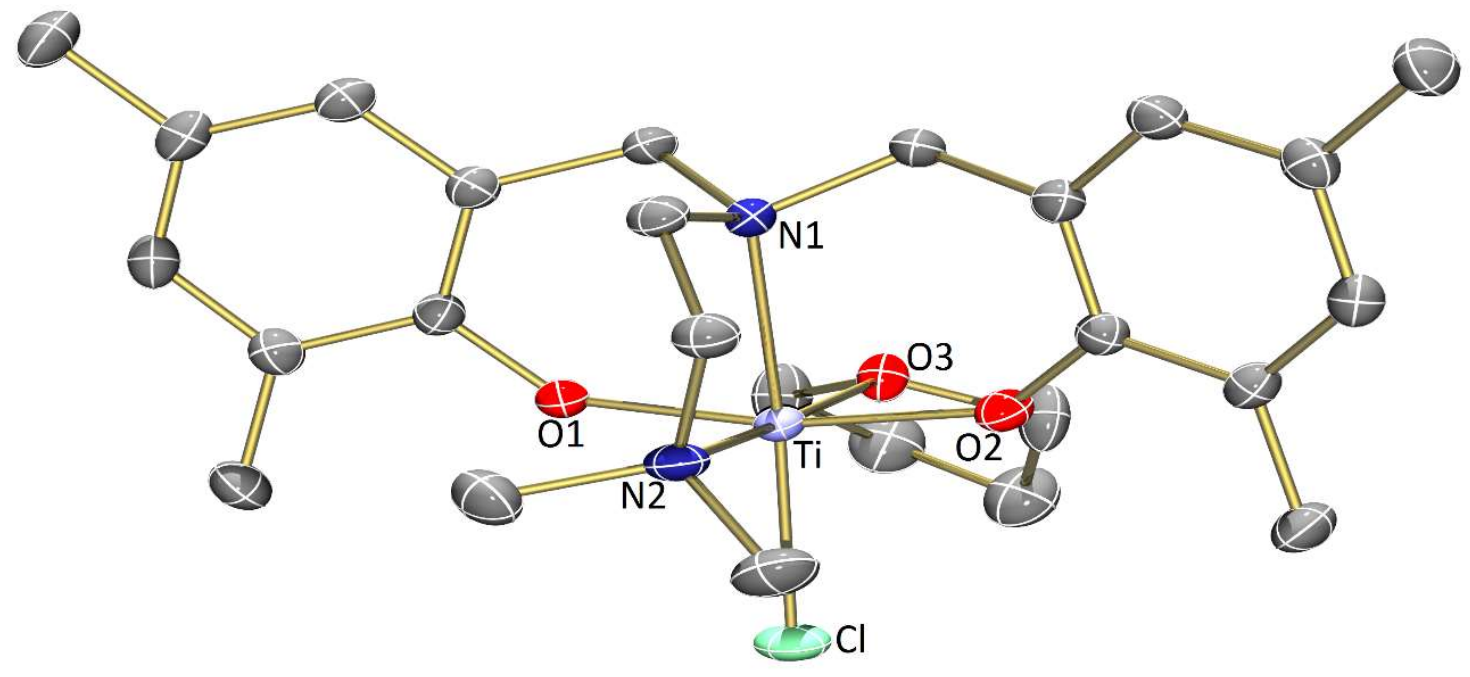

CCDC code

Formula

Formula weight

Size

Crystal morphology

Temperature

Wavelength

Crystal system

Space group

Unit cell dimensions

Volume

Z

Density (calculated)

Absorption coefficient

$F(000)$

Data collection range

Index ranges

Reflections collected
1889717

$\mathrm{C}_{56} \mathrm{H}_{84} \mathrm{Cl}_{2} \mathrm{~N}_{4} \mathrm{O}_{7} \mathrm{Ti}_{2}$

1091.91

$0.2663 \times 0.0972 \times 0.0344 \mathrm{~mm}$

Yellow plate

$120.00(10) \mathrm{K}$

$1.54184 \AA\left[\mathrm{Cu}-K_{\alpha}\right]$

Monoclinic

$P 21 / C$

$a=8.61539(7) \AA$

$\alpha=90^{\circ}$

$b=15.80257(11) \AA$

$\beta=96.8755(8)^{\circ}$

$c=20.58955(17) \AA$

$\gamma=90^{\circ}$

2783.01(4) $\AA^{3}$

2

$1.303 \mathrm{Mg} / \mathrm{m}^{3}$

$3.751 \mathrm{~mm}^{-1}$

1164

$3.535 \leq \theta \leq 76.187^{\circ}$

$-10 \leq h \leq 10,-19 \leq k \leq 16,-25 \leq I \leq 25$

44749 
Independent reflections

Observed reflections

Absorption correction

Max. and min. transmission

Refinement method

Data / restraints / parameters

Goodness of fit

Final $R$ indices $[I>2 \sigma(I)]$

$R$ indices (all data)

Largest diff. peak and hole
$5809[R$ (int $)=0.0718]$

$5526[I>2 \sigma(I)]$

multi-scan

1 and 0.62598

Full

5809 / 3 / 346

1.042

$R_{1}=0.0491, w R_{2}=0.1365$

$R_{1}=0.0507, w R_{2}=0.1386$

0.604 and $-0.551 e . \AA^{-3}$

Figure S25 Molecular structure of $\mathbf{2}(\boldsymbol{\mu}-\mathrm{Cl})_{2}$ with ellipsoids set at the $50 \%$ probability level. Hydrogen atoms have been omitted for clarity.

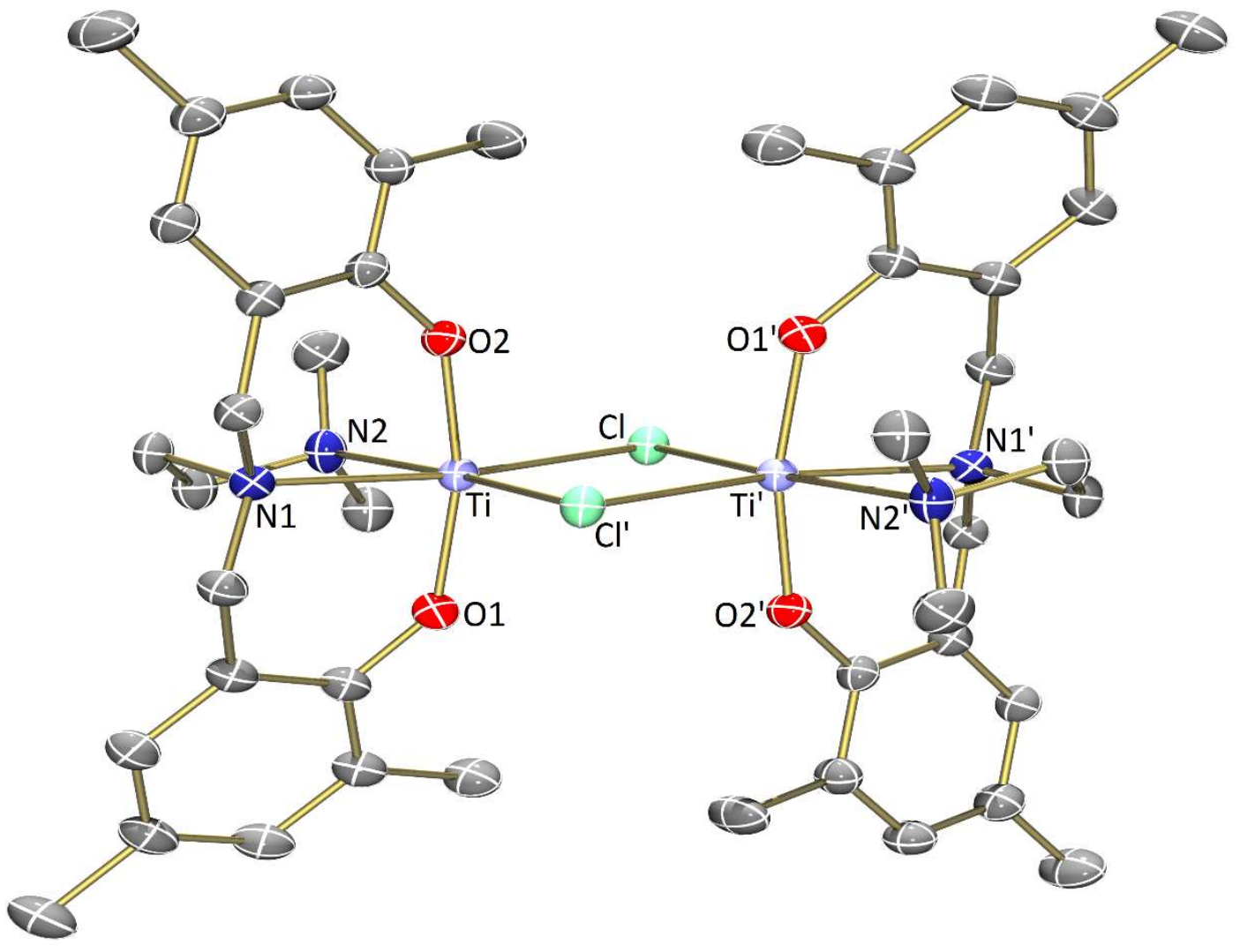

CCDC code

Formula

Formula weight

Size

Crystal morphology

Temperature

Wavelength

Crystal system

Space group
1889718

$\mathrm{C}_{44} \mathrm{H}_{60} \mathrm{Cl}_{2} \mathrm{~N}_{4} \mathrm{O}_{4} \mathrm{Ti}_{2}$

875.66

$0.217 \times 0.0685 \times 0.0393 \mathrm{~mm}$

Light green prism

120.00(10) K

$1.54184 \AA$ A [Cu- $\left.K_{\alpha}\right]$

Monoclinic

$P 21 / n$ 
Unit cell dimensions

Volume

Z

Density (calculated)

Absorption coefficient

$F(000)$

Data collection range

Index ranges

Reflections collected

Independent reflections

Observed reflections

Absorption correction

Max. and min. transmission

Refinement method

Data / restraints / parameters

Goodness of fit

Final $R$ indices $[I>2 \sigma(I)]$

$R$ indices (all data)

Largest diff. peak and hole

$$
\begin{array}{ll}
a=8.24598(11) \AA & \alpha=90^{\circ} \\
b=19.1270(3) \AA & \beta=90.3456(14)^{\circ} \\
c=13.8373(2) \AA & \gamma=90^{\circ}
\end{array}
$$

2182.38(6) $\AA^{3}$

2

$1.333 \mathrm{Mg} / \mathrm{m}^{3}$

$4.599 \mathrm{~mm}^{-1}$

924

$3.943 \leq \theta \leq 76.546^{\circ}$

$-8 \leq h \leq 10,-24 \leq k \leq 23,-17 \leq l \leq 17$

44176

$4553[R($ int $)=0.0781]$

$4270[I>2 \sigma(I)]$

multi-scan

1 and 0.51912

Full

4553 / 0 / 259

1.066

$R_{1}=0.0481, w R_{2}=0.1325$

$R_{1}=0.0501, w R_{2}=0.1347$

0.856 and $-0.565 e . \AA^{-3}$

Figure S26 Molecular structure of $\mathbf{3}$ with ellipsoids set at the $50 \%$ probability level. Hydrogen atoms and minor disorder component have been omitted for clarity.

Note. One carbon atom ('C21') of the coordinated THF molecule was found to be disordered over two positions. The two modelled sites (C21A and C21B) could be refined satisfactorily with SOFs of 0.616 and 0.384 respectively.

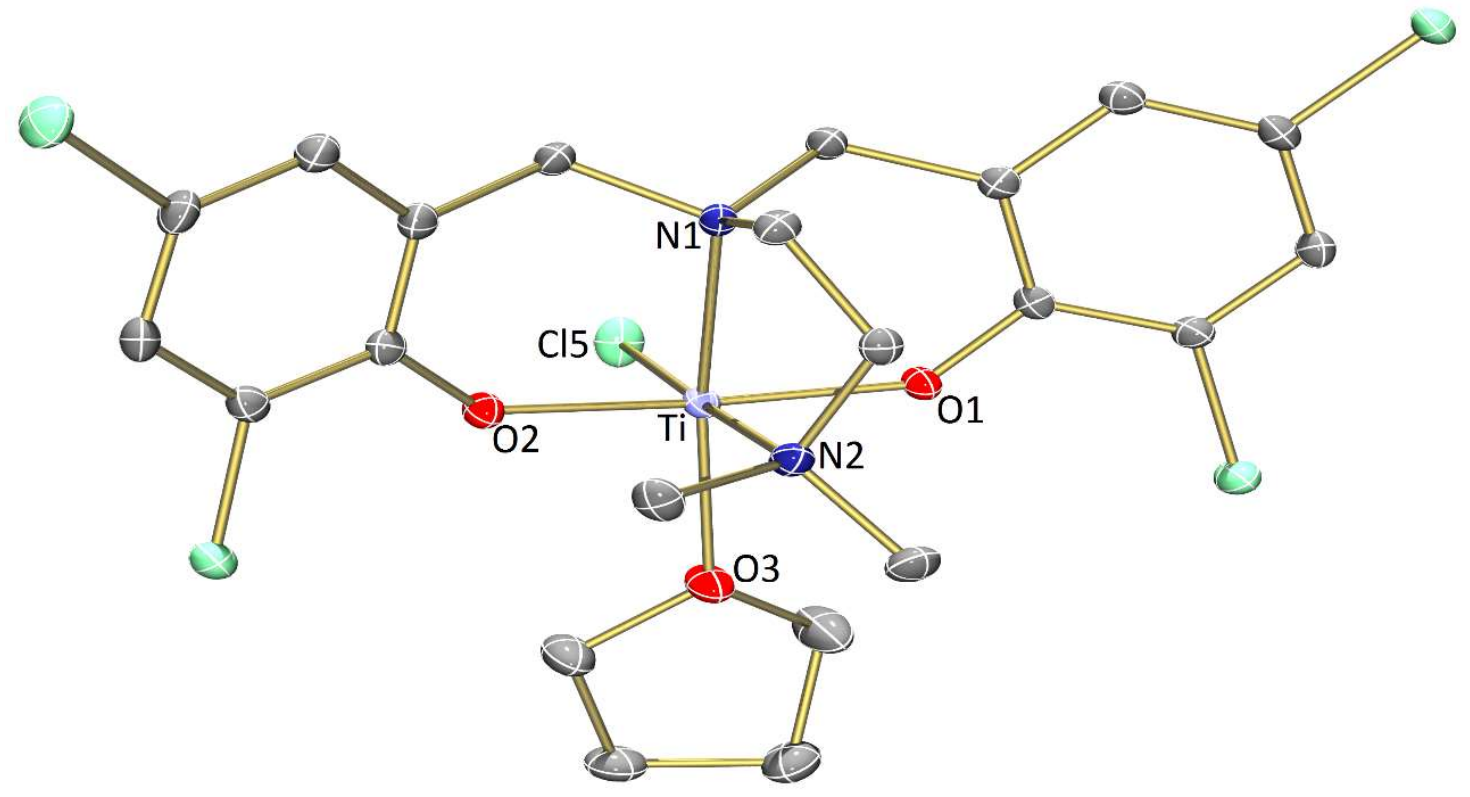


CCDC code

Formula

Formula weight

Size

Crystal morphology

Temperature

Wavelength

Crystal system

Space group

Unit cell dimensions

Volume

Z

Density (calculated)

Absorption coefficient

$F(000)$

Data collection range

Index ranges

Reflections collected

Independent reflections

Observed reflections

Absorption correction

Max. and min. transmission

Refinement method

Data / restraints / parameters

Goodness of fit

Final $R$ indices $[I>2 \sigma(I)]$

$R$ indices (all data)

Largest diff. peak and hole
1889719

$\mathrm{C}_{22} \mathrm{H}_{26} \mathrm{Cl}_{5} \mathrm{~N}_{2} \mathrm{O}_{3} \mathrm{Ti}$

591.6

$0.3517 \times 0.1178 \times 0.0475 \mathrm{~mm}$

Yellow prism

$120.00(10) \mathrm{K}$

$0.71073 \AA ̊\left[\mathrm{Mo}-K_{\alpha}\right.$ ]

Orthorhombic

Pbca

$\begin{array}{ll}a=15.9365(3) \AA & \alpha=90^{\circ} \\ b=14.9569(2) \AA & \beta=90^{\circ} \\ c=21.2219(3) \AA & \gamma=90^{\circ}\end{array}$

5058.45(14) $\AA^{3}$

8

$1.554 \mathrm{Mg} / \mathrm{m}^{3}$

$0.894 \mathrm{~mm}^{-1}$

2424

$2.888 \leq \theta \leq 32.959^{\circ}$

$-24 \leq h \leq 24,-22 \leq k \leq 22,-32 \leq I \leq 32$

248707

$9356[R$ (int $)=0.071]$

$8471[I>2 \sigma(I)]$

multi-scan

1 and 0.78809

Full

$9356 / 0$ / 310

1.223

$R_{1}=0.0473, w R_{2}=0.0869$

$R_{1}=0.0575, w R_{2}=0.0899$

0.634 and $-0.356 e . \AA^{-3}$

Figure S27 Molecular structure of $\mathbf{3}(\mu-\mathbf{C l})_{2}$ with ellipsoids set at the $50 \%$ probability level. Hydrogen atoms and disordered solvent have been omitted for clarity.

Note. Significant residual electron density in the vicinity of the Ti complex could be refined as 1 molecule of toluene with full positional disorder over two positions. Assignment of SOFs of 0.65 and 0.35 respectively for the two independent molecules gave stable refinement and convergence. It was necessary to refine the minor component as a rigid hexagon to ensure a chemically sensible structure was obtained. 


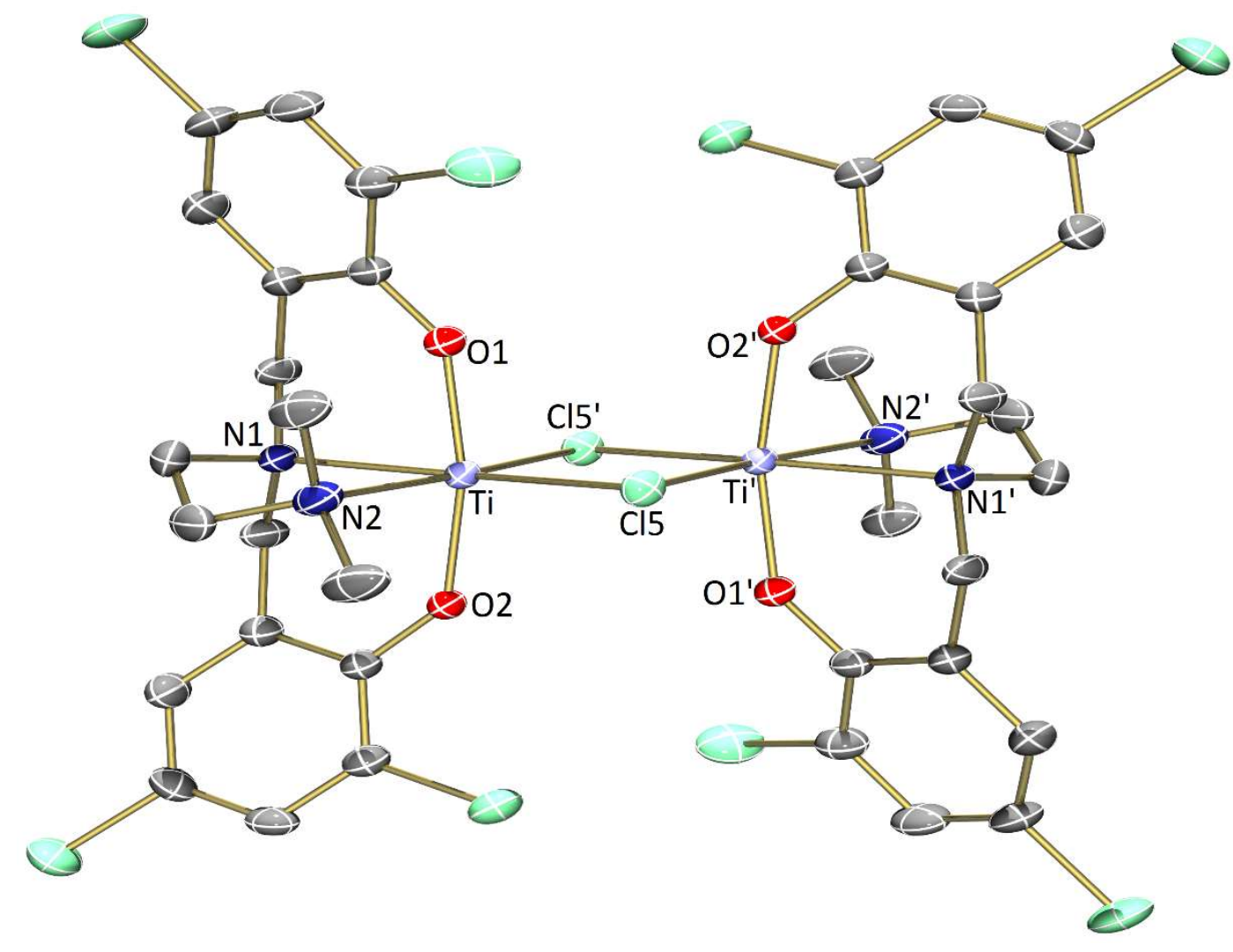

CCDC code

Formula

Formula weight

Size

Crystal morphology

Temperature

Wavelength

Crystal system

Space group

Unit cell dimensions

Volume

Z

Density (calculated)

Absorption coefficient

$F(000)$

Data collection range

Index ranges

Reflections collected

Independent reflections
1889720

$\mathrm{C}_{50} \mathrm{H}_{52} \mathrm{Cl}_{10} \mathrm{~N}_{4} \mathrm{O}_{4} \mathrm{Ti}_{2}$

1223.25

$0.3408 \times 0.2758 \times 0.046 \mathrm{~mm}$

Green plate

120.00(10) K

$0.71073 \AA$ [Mo- $K_{\alpha}$ ]

Monoclinic

$\mathrm{C} 2 / \mathrm{c}$

$a=15.1807(5) \AA$

$\alpha=90^{\circ}$

$b=19.7102(6) \AA$

$\beta=98.694(4)^{\circ}$

$c=18.0932(7) \AA$

$\gamma=90^{\circ}$

5351.5(3) $\AA^{3}$

4

$1.518 \mathrm{Mg} / \mathrm{m}^{3}$

$0.846 \mathrm{~mm}^{-1}$

2504

$3.076 \leq \theta \leq 29.613^{\circ}$

$-20 \leq h \leq 21,-26 \leq k \leq 25,-24 \leq I \leq 24$

47257

$7083[R$ (int $)=0.0677]$ 
Observed reflections

Absorption correction

Max. and min. transmission

Refinement method

Data / restraints / parameters

Goodness of fit

Final $R$ indices $[I>2 \sigma(I)]$

$R$ indices (all data)

Largest diff. peak and hole
$5393[I>2 \sigma(I)]$

gaussian

0.953 and 0.743

Full

7083 / 0 / 371

1.088

$R_{1}=0.0541, w R_{2}=0.0924$

$R_{1}=0.0813, w R_{2}=0.1014$

0.435 and $-0.4 e . \AA^{-3}$

Figure S28 Molecular structures 4 with ellipsoids set at the $50 \%$ probability level. Hydrogen atoms, disordered solvent and minor disorder components have been omitted for clarity.

Note. Three atoms of the pendant THF donor (C32, C33 \& C34) were found to be disordered over two positions, and were successfully modelled with a SOF of 0.652 (C32A, C33A \& C34A) for the major component and 0.348 (C32B, C33B \& C34B) for the minor component. The disorder likely originates from the use of a racemic starting material (tetrahydrofurfurylamine) and thus due to the cocrystallization of the two possible enantiomers ( $R$ and $S)$. In addition, further residual electron density could be modelled as a molecule of toluene, which was found to be positionally disordered over an inversion centre. Thus, the toluene was modelled with a PART -1 command and a SOF of 0.5 .

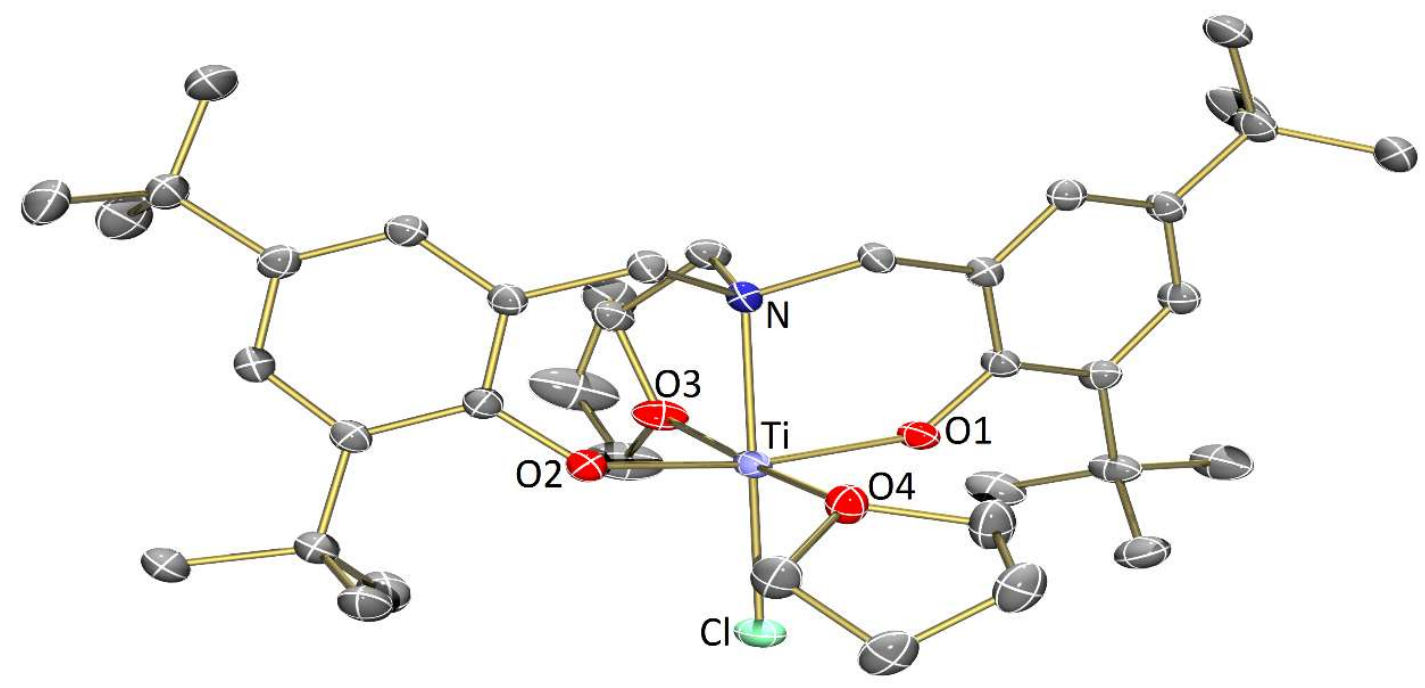

CCDC code

Formula

Formula weight

Size

Crystal morphology

Temperature

Wavelength

Crystal system

Space group

Unit cell dimensions
1889721

$\mathrm{C}_{85} \mathrm{H}_{130} \mathrm{Cl}_{2} \mathrm{~N}_{2} \mathrm{O}_{8} \mathrm{Ti}_{2}$

1474.6

$0.661 \times 0.12 \times 0.023 \mathrm{~mm}$

Colourless plate

120.01(10) K

$1.54184 \AA\left[\mathrm{Cu}-K_{\alpha}\right]$

Monoclinic

$P 21 / c$

$a=12.7740(4) \AA \quad \alpha=90^{\circ}$ 


\begin{tabular}{|c|c|c|}
\hline & $b=20.1276(6) \AA$ & $\beta=94.834(3)^{\circ}$ \\
\hline & $c=16.5375(4) \AA$ & $\gamma=90^{\circ}$ \\
\hline Volume & $4236.8(2) \AA^{3}$ & \\
\hline$z$ & 2 & \\
\hline Density (calculated) & $1.156 \mathrm{Mg} / \mathrm{m}^{3}$ & \\
\hline Absorption coefficient & $2.586 \mathrm{~mm}^{-1}$ & \\
\hline$F(000)$ & 1592 & \\
\hline Data collection range & $3.466 \leq \theta \leq 76.10$ & \\
\hline Index ranges & $-15 \leq h \leq 16,-25 \leq$ & $10 \leq I \leq 20$ \\
\hline Reflections collected & 34874 & \\
\hline Independent reflections & $8785[R(\mathrm{int})=0.0$ & \\
\hline Observed reflections & $7216[I>2 \sigma(I)]$ & \\
\hline Absorption correction & gaussian & \\
\hline Max. and min. transmission & 1 and 0.348 & \\
\hline Refinement method & Full & \\
\hline Data / restraints / parameters & 8785 / 4 / 519 & \\
\hline Goodness of fit & 1.038 & \\
\hline Final $R$ indices $[/>2 \sigma(/)]$ & $R_{1}=0.0567, w R_{2}$ & \\
\hline$R$ indices (all data) & $R_{1}=0.069, w R_{2}=$ & \\
\hline Largest diff. peak and hole & 0.466 and -0.721 & \\
\hline
\end{tabular}

Figure S29 Molecular structure of 6 with ellipsoids set at the 50\% probability level. Hydrogen atoms and the minor disorder component have been omitted for clarity.

Note. This complex is structurally analogous to a vanadium(III) complex reported by Goldschmidt, Kol and coworkers (Inorg. Chem., 2005, 44 (14), pp 5073-5080). In this report, the overall quality of the reported structure was low, which was explained to be mainly due to twinning effects. For our dataset, no suitable twin laws could be discerned. Rather, the structure was simply found to be heavily positionally disordered (40\% disorder) over two positions. While the donor THF, Ti, N atom and most of the tert-butyl groups were common to both components of the disorder, most of the phenolate ring atoms were disordered, as were the benzylic carbon atoms and phenolate $O$ atoms. Free refinement of the two disorder components (which were both clear in the diff. map) gave convergence at SOFs of 0.61 and 0.39 respectively (for the major and minor components, PART 1 and PART 2). Use of SADI restraints on the disordered components ensured that bond parameters were chemically sensible. In one case, it was unfortunately necessary to use an EADP constraint (since the use of softer SIMU, DELU, ISOR constraints was ineffective). Additionally, one of the tert-butyl groups was found to be disordered over two positions. Refinement of the two disorder components with SOFs of 0.72 and 0.28 gave a satisfactory outcome. 


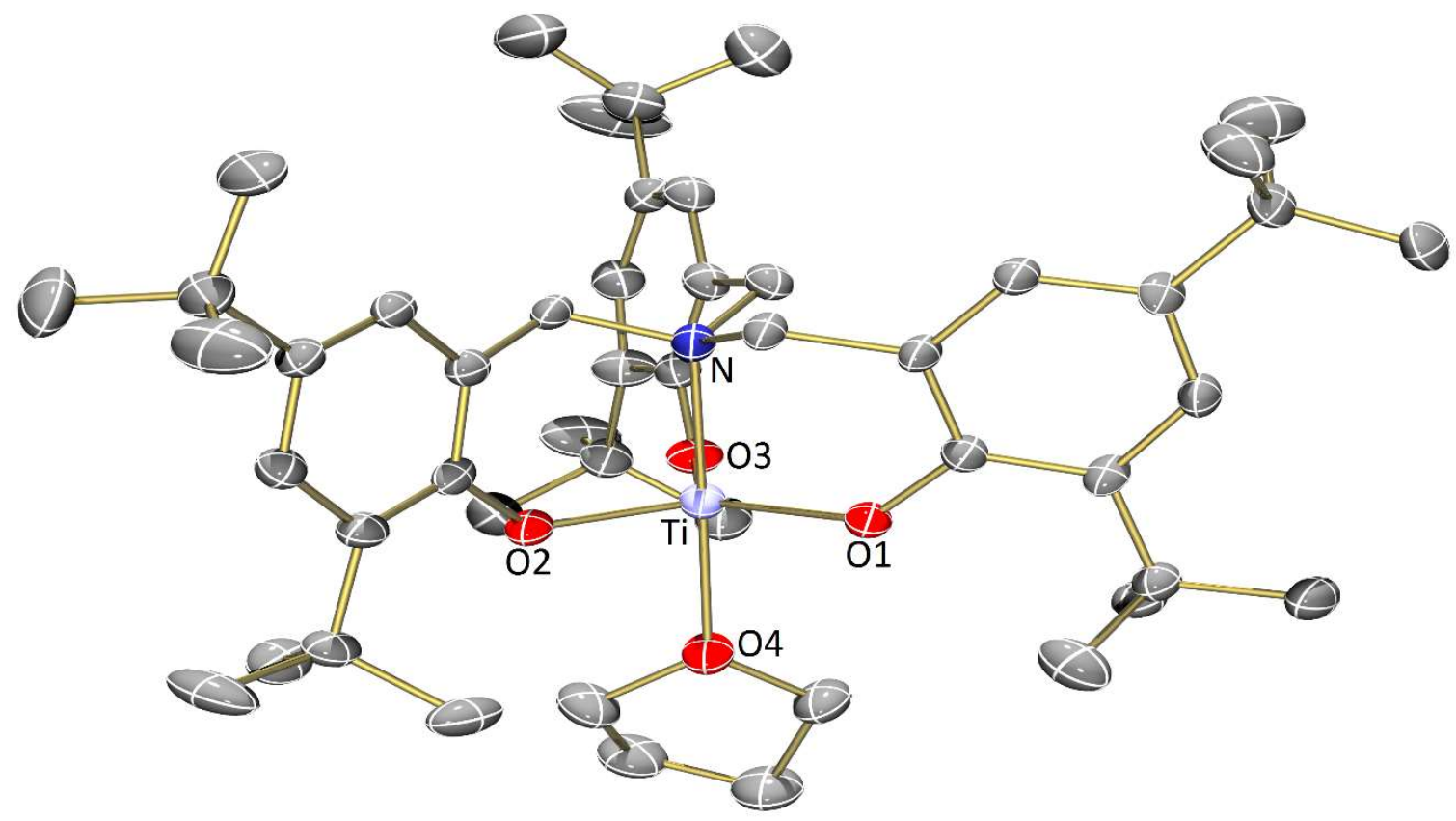

Figure S30 Molecular structures of 6 viewed along the $\mathrm{N}$-Ti bond with ellipsoids set at the $50 \%$ probability level illustrating the a) major and b) minor components of the disorder and c) the superimposition of both components. Hydrogen atoms and the coordinated THF (which is common to both components) have been omitted for clarity.

a)

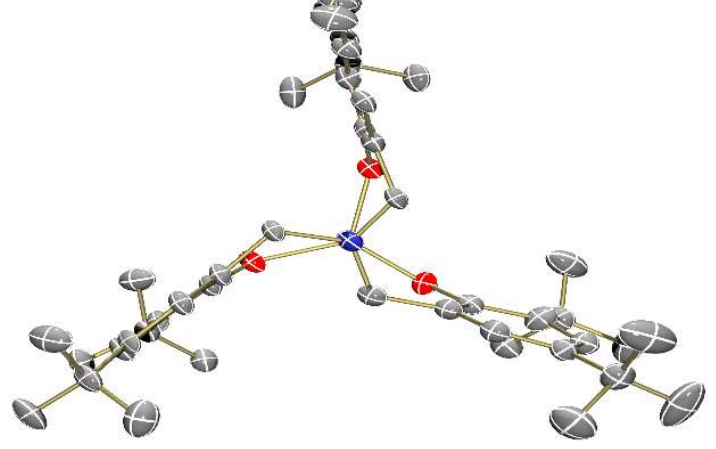

b)

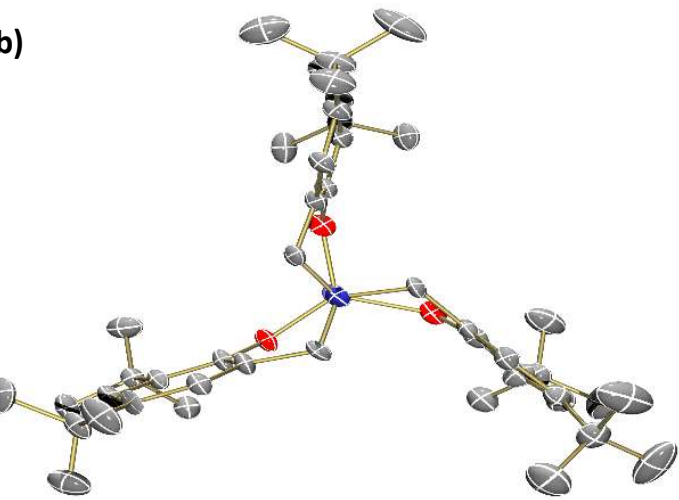

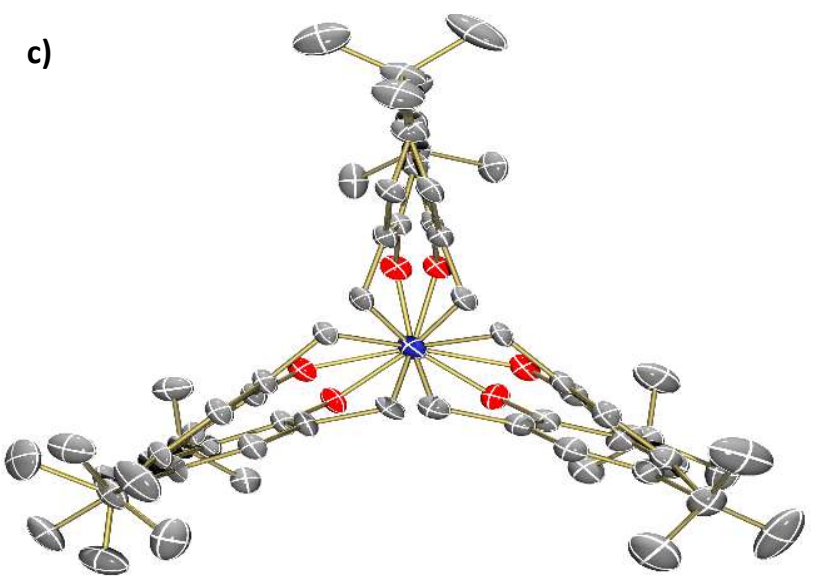


CCDC code

Formula

Formula weight

Size

Crystal morphology

Temperature

Wavelength

Crystal system

Space group

Unit cell dimensions

Volume

Z

Density (calculated)

Absorption coefficient

$F(000)$

Data collection range

Index ranges

Reflections collected

Independent reflections

Observed reflections

Absorption correction

Max. and min. transmission

Refinement method

Data / restraints / parameters

Goodness of fit

Final $R$ indices $[I>2 \sigma(I)]$

$R$ indices (all data)

Largest diff. peak and hole
1889722

$\mathrm{C}_{49} \mathrm{H}_{74} \mathrm{NO}_{4} \mathrm{Ti}$

788.99

$0.263 \times 0.105 \times 0.033 \mathrm{~mm}$

Colourless plate

120.01(10) K

$1.54184 \AA\left[\mathrm{Cu}-K_{\alpha}\right]$

Triclinic

$P-1$

$\begin{array}{ll}a=10.3781(2) \AA & \alpha=91.943(2)^{\circ} \\ b=11.0454(2) \AA & \beta=100.093(2)^{\circ} \\ c=20.6189(5) \AA & \gamma=96.4710(10)^{\circ}\end{array}$

2308.67(8) $\AA^{3}$

2

$1.135 \mathrm{Mg} / \mathrm{m}^{3}$

$1.884 \mathrm{~mm}^{-1}$

858

$4.034 \leq \theta \leq 76.395^{\circ}$

$-11 \leq h \leq 12,-13 \leq k \leq 13,-25 \leq l \leq 25$

44555

$9466[R$ (int) $=0.0703]$

$8262[I>2 \sigma(I)]$

gaussian

1 and 0.653

Full

9466 / 14 / 711

1.087

$R_{1}=0.068, w R_{2}=0.1763$

$R_{1}=0.0761, w R_{2}=0.183$

0.755 and $-0.624 e . \AA^{-3}$ 


\section{COMPUTATIONAL DATA}

Table S5 Benchmarking of three functionals against published crystal structure. ${ }^{10}$

\begin{tabular}{cccccc}
\hline Bond/Angle & Structure 1 & Structure 2 & B3LYP & BP86 & M06 \\
\hline Ti-Cl & 2.41831 & 2.41862 & 2.40054 & 2.39643 & 2.37623 \\
Ti-O1 & 1.90541 & 1.89781 & 1.91068 & 1.91708 & 1.90251 \\
Ti-O2 & 1.90628 & 1.90855 & 1.91652 & 1.92144 & 1.91521 \\
Ti-O(THF) & 2.22565 & 2.22425 & 2.25951 & 2.26529 & 2.21958 \\
Ti-N(pivot) & 2.27749 & 2.27879 & 2.40697 & 2.3897 & 2.36881 \\
Ti-N(arm) & 2.28153 & 2.28388 & 2.33739 & 2.32005 & 2.2939 \\
& & & & & 173.051 \\
Cl-Ti-N(pivot) & 173.946 & 173.835 & 173.08 & 173.808 \\
O1-Ti-O2 & 167.546 & 167.453 & 162.884 & 163.762 & 164.258 \\
N(arm)-Ti-O(THF) & 172.251 & 172.33 & 176.281 & 176.528 & 176.441 \\
\hline
\end{tabular}

Cartesian coordinates in $\AA$ and energies in hartrees for all optimized geometries. E values are electronic energies in the gas phase. $\mathrm{G}$ values are at $298 \mathrm{~K}$ and adjusted for $1 \mathrm{M}$ solution.

\begin{tabular}{|c|c|c|c|c|c|c|c|}
\hline \multirow{2}{*}{\multicolumn{2}{|c|}{ Organic Molecules }} & & & 1 & 2.784537 & -0.805676 & -0.917766 \\
\hline & & & & 1 & 2.866194 & -0.729277 & 0.841817 \\
\hline \multicolumn{4}{|c|}{$\mathrm{CH}_{2}=\mathrm{C}\left(\mathrm{CH}_{3}\right) \mathrm{COOCH}_{3}$} & 1 & 1.769942 & -1.913019 & 0.055736 \\
\hline \multicolumn{4}{|c|}{$E=-345.308729$} & 6 & 1.445511 & 1.556544 & 0.000057 \\
\hline \multicolumn{4}{|c|}{$G=-345.216117$} & 1 & 2.064750 & 1.805832 & 0.882804 \\
\hline \multirow{6}{*}{\multicolumn{2}{|c|}{80}} & & & 1 & 2.073921 & 1.799141 & -0.878030 \\
\hline & & & & 1 & 0.569034 & 2.215662 & -0.006650 \\
\hline & & & & 6 & -0.292738 & -0.354032 & 0.001932 \\
\hline & & & & 8 & -0.624787 & -1.521434 & 0.001819 \\
\hline & & & & 8 & -1.194955 & 0.652073 & 0.000222 \\
\hline & & 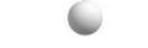 & & 6 & -2.548755 & 0.255617 & -0.002097 \\
\hline 6 & 1.139370 & 0.256386 & -0.000033 & 1 & -2.787424 & -0.346353 & -0.893751 \\
\hline 6 & 1.356526 & 1.576706 & 0.000029 & 1 & -2.788618 & -0.352843 & 0.884765 \\
\hline 1 & 2.376432 & 1.974434 & 0.000120 & 1 & -3.149944 & 1.173554 & 0.000620 \\
\hline 1 & 0.530957 & 2.291572 & 0.000027 & & & & \\
\hline 6 & 2.223700 & -0.769501 & 0.000028 & & & & \\
\hline 1 & 2.143145 & -1.429166 & -0.879326 & \multicolumn{4}{|c|}{$\left(\mathrm{CH}_{3}\right)_{2} \mathrm{C}^{\bullet}(\mathrm{CN})$} \\
\hline 1 & 3.217114 & -0.298544 & 0.000731 & \multicolumn{4}{|c|}{$E=-210.413718$} \\
\hline 1 & 2.142217 & -1.430069 & 0.878604 & \multicolumn{2}{|c|}{$G=-210.353582$} & & \\
\hline 6 & -0.242165 & -0.300893 & -0.000052 & \multirow{2}{*}{\multicolumn{2}{|c|}{$e^{20000}$}} & & \\
\hline 8 & -0.484435 & -1.482326 & 0.000011 & & & & \\
\hline 8 & -1.198898 & 0.637991 & -0.000103 & & & & \\
\hline 6 & -2.528446 & 0.158470 & 0.000081 & & & & \\
\hline 1 & -2.727502 & -0.458069 & -0.890645 & & & & \\
\hline 1 & -2.726298 & -0.460818 & 0.889140 & \multirow{2}{*}{\multicolumn{2}{|c|}{68}} & & \\
\hline \multirow[t]{2}{*}{1} & \multirow[t]{2}{*}{-3.183310} & 1.038337 & 0.001768 & & & -0.000008 & -0.000004 \\
\hline & & & & 6 & -1.063292 & -1.285093 & -0.000007 \\
\hline \multicolumn{4}{|c|}{$\left(\mathrm{CH}_{3}\right)_{2} \mathrm{C}^{\bullet}\left(\mathrm{COOCH}_{3}\right)$} & 1 & -1.724014 & -1.349002 & 0.884195 \\
\hline \multicolumn{4}{|c|}{$E=-345.889640$} & 1 & -1.724121 & -1.348936 & -0.884133 \\
\hline \multirow{2}{*}{\multicolumn{4}{|c|}{$G=-345.789905$}} & 1 & -0.399666 & -2.160662 & -0.000075 \\
\hline & & & & 6 & -1.063196 & 1.285141 & -0.000009 \\
\hline \multirow{5}{*}{\multicolumn{2}{|c|}{8}} & & & 1 & -1.723954 & 1.349072 & -0.884182 \\
\hline & & & & 1 & -1.723976 & 1.349066 & 0.884148 \\
\hline & & & & 1 & -0.399497 & 2.160654 & 0.000003 \\
\hline & & & & 6 & 1.085839 & -0.000032 & 0.000012 \\
\hline & & & & 7 & 2.257868 & -0.000034 & 0.000013 \\
\hline 6 & 1.085778 & 0.118318 & 0.003993 & \multirow{2}{*}{\multicolumn{4}{|c|}{$\left(\mathrm{CH}_{2}\right)_{4} \mathrm{O}$}} \\
\hline 6 & 2.169461 & -0.891803 & -0.001531 & & & & \\
\hline
\end{tabular}




$\begin{array}{rrrr}G=-232.021355 & & \\ & & & \\ & & & \\ & & & \\ & & & \\ 1 & -1.599226 & -1.072320 & 0.460964 \\ 8 & 0.000000 & 0.000000 & 1.232695 \\ 6 & 0.489784 & 1.050391 & 0.426978 \\ 6 & 0.000000 & 0.760617 & -0.982401 \\ 6 & 0.000000 & -0.760617 & -0.982401 \\ 6 & -0.489784 & -1.050391 & 0.426978 \\ 1 & 0.132904 & 2.017333 & 0.823091 \\ 1 & 1.599226 & 1.072320 & 0.460964 \\ 1 & 0.629975 & 1.211324 & -1.764333 \\ 1 & -1.028246 & 1.140582 & -1.117962 \\ 1 & 1.028246 & -1.140582 & -1.117962 \\ 1 & -0.629975 & -1.211324 & -1.764333 \\ 1 & -0.132904 & -2.017333 & 0.823091\end{array}$


Complexes in Scheme S3
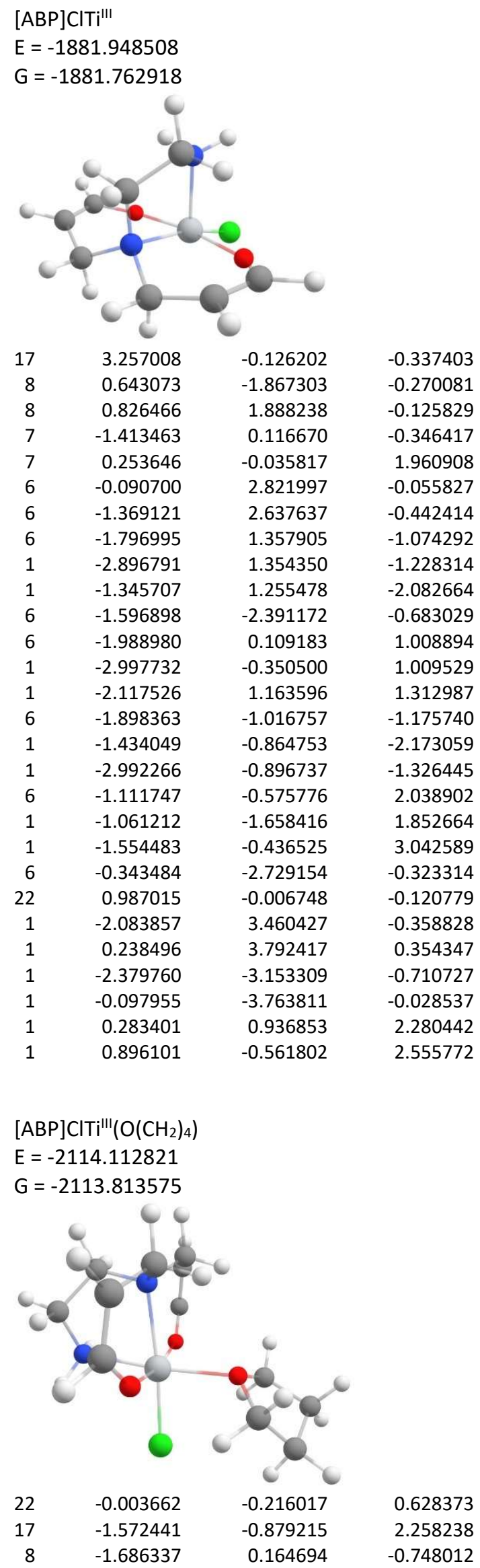

$\begin{array}{rrrr}8 & 0.040906 & 1.652791 & 1.086569 \\ 8 & 0.302381 & -1.965442 & -0.111981 \\ 7 & 1.726901 & 0.418330 & -0.867514 \\ 7 & 1.822097 & -0.476864 & 1.879395 \\ 6 & 0.694389 & 2.739903 & 0.766801 \\ 6 & 1.140089 & -2.573816 & -0.913730 \\ 6 & 1.426022 & 2.877496 & -0.358314 \\ 6 & 1.726535 & -0.468081 & -2.059935 \\ 1 & 2.503836 & -0.102818 & -2.764858 \\ 1 & 0.750276 & -0.277895 & -2.552883 \\ 6 & 1.900715 & -1.930237 & -1.821576 \\ 6 & 1.492792 & 1.795880 & -1.382674 \\ 1 & 0.545795 & 1.732218 & -1.955781 \\ 1 & 2.282113 & 2.031887 & -2.128521 \\ 6 & 2.989220 & 0.354710 & -0.108512 \\ 1 & 3.845148 & 0.163790 & -0.787843 \\ 1 & 3.147986 & 1.356220 & 0.331085 \\ 6 & 2.993965 & -0.665522 & 1.017623 \\ 1 & 3.945962 & -0.573397 & 1.573834 \\ 1 & 2.956606 & -1.686366 & 0.610917 \\ 6 & -3.915191 & -0.565381 & -0.782497 \\ 1 & -4.111507 & -0.769512 & 0.284110 \\ 1 & -4.664100 & -1.101156 & -1.384519 \\ 6 & -2.496073 & -0.973719 & -1.112714 \\ 1 & -2.143178 & -1.846866 & -0.544614 \\ 1 & -2.350327 & -1.163474 & -2.192540 \\ 6 & -2.528893 & 1.296358 & -0.447017 \\ 1 & -2.574735 & 1.413442 & 0.650358 \\ 1 & -2.062948 & 2.196676 & -0.878090 \\ 6 & -3.877305 & 0.935376 & -1.028203 \\ 1 & -3.910634 & 1.154468 & -2.110392 \\ 1 & -4.699940 & 1.484587 & -0.546327 \\ 1 & 2.588008 & -2.496773 & -2.455333 \\ 1 & 1.221516 & -3.668324 & -0.790427 \\ 1 & 0.632821 & 3.571352 & 1.491567 \\ 1 & 1.949197 & 3.818981 & -0.546226 \\ 1 & 1.897094 & 0.372780 & 2.444345 \\ 1 & 1.6868886 & -1.255211 & 2.526597\end{array}$

[ABP]ClTi"II $\left(\mathrm{OC}\left(\mathrm{OCH}_{3}\right) \mathrm{C}\left(\mathrm{CH}_{2}\right)\left(\mathrm{CH}_{3}\right)\right)$

$E=-2227.306852$

$G=-2227.005299$

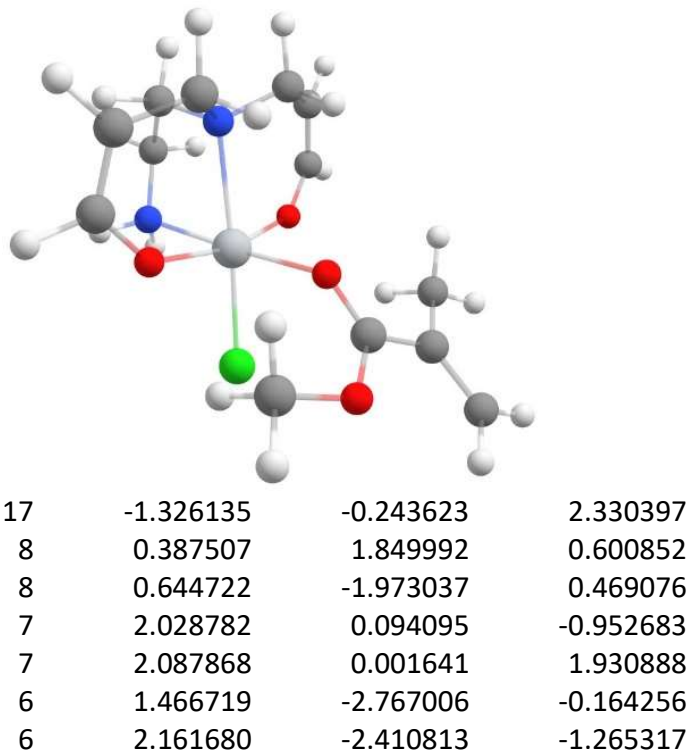




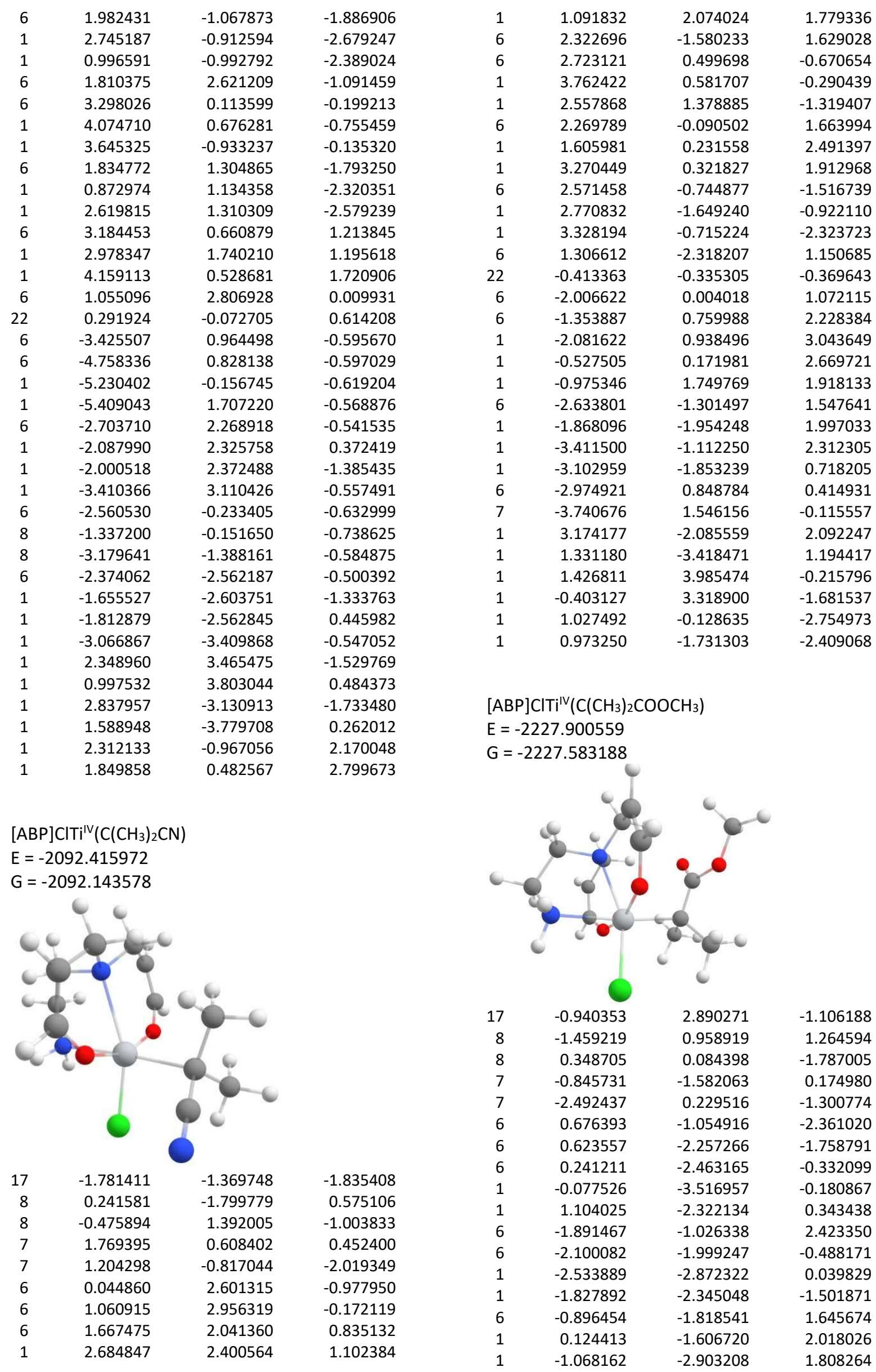




$\begin{array}{rrrr}6 & -3.128417 & -0.904879 & -0.643626 \\ 1 & -3.509987 & -0.574396 & 0.332818 \\ 1 & -3.992185 & -1.303585 & -1.209147 \\ 6 & -2.068739 & 0.290083 & 2.223764 \\ 22 & -0.469607 & 0.828958 & -0.296967 \\ 6 & 1.477443 & 1.212750 & 0.694153 \\ 6 & 2.162860 & 2.263470 & -0.182429 \\ 1 & 2.177959 & 1.999798 & -1.250798 \\ 1 & 1.659682 & 3.236585 & -0.081148 \\ 1 & 3.215869 & 2.395035 & 0.133000 \\ 6 & 1.201708 & 1.762052 & 2.088042 \\ 1 & 0.771861 & 1.005044 & 2.761362 \\ 1 & 2.139763 & 2.124966 & 2.553536 \\ 1 & 0.504050 & 2.614524 & 2.046310 \\ 6 & 2.241857 & -0.043971 & 0.767869 \\ 8 & 2.169852 & -0.877458 & 1.655111 \\ 8 & 3.074564 & -0.223627 & -0.280777 \\ 6 & 3.853149 & -1.397271 & -0.288421 \\ 1 & 4.334734 & -1.567618 & 0.686806 \\ 1 & 3.240658 & -2.282540 & -0.533265 \\ 1 & 4.615462 & -1.267620 & -1.068015 \\ 1 & 0.918151 & -3.134723 & -2.342055 \\ 1 & 0.998466 & -0.971286 & -3.411054 \\ 1 & -2.427267 & -1.514955 & 3.241508 \\ 1 & -2.761286 & 0.880110 & 2.843868 \\ 1 & -2.285603 & 0.035868 & -2.283660 \\ 1 & -3.066095 & 1.072913 & -1.273008\end{array}$

$\begin{array}{rrrr}22 & 0.694241 & -0.149327 & 0.853193 \\ 6 & -3.190592 & 0.752888 & 0.166996 \\ 6 & -4.544841 & 0.654064 & -0.451896 \\ 1 & -4.731691 & -0.334932 & -0.890903 \\ 1 & -5.333616 & 0.860382 & 0.295091 \\ 1 & -4.674046 & 1.413357 & -1.245696 \\ 6 & -2.822050 & 2.060291 & 0.785414 \\ 1 & -1.866362 & 2.016100 & 1.326881 \\ 1 & -2.744436 & 2.857288 & 0.020465 \\ 1 & -3.604437 & 2.390387 & 1.492655 \\ 6 & -2.324052 & -0.286306 & 0.157011 \\ 8 & -1.124148 & -0.248121 & 0.710920 \\ 8 & -2.619509 & -1.441281 & -0.500472 \\ 6 & -2.375893 & -2.634094 & 0.214271 \\ 1 & -1.311937 & -2.745186 & 0.479069 \\ 1 & -2.977301 & -2.667471 & 1.141353 \\ 1 & -2.675659 & -3.467153 & -0.435810 \\ 1 & 0.441733 & 3.666918 & -1.740274 \\ 1 & 0.971405 & 3.754445 & 0.645500 \\ 1 & 0.999462 & -2.866947 & -2.880567 \\ 1 & 1.607904 & -3.648678 & -0.657246 \\ 1 & 3.283141 & -0.855737 & 0.386458 \\ 1 & 3.286836 & 0.513849 & 1.302482\end{array}$
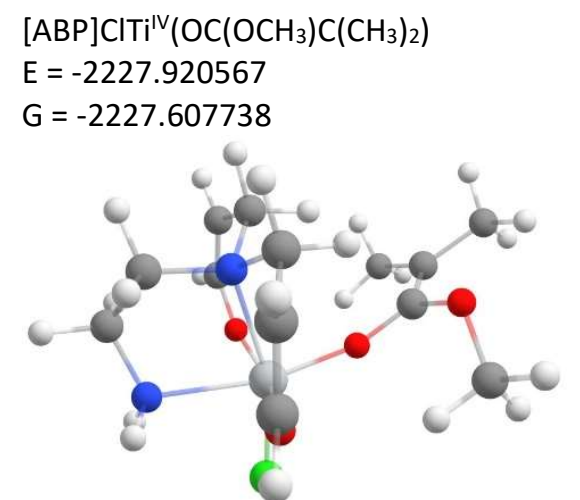

$\begin{array}{rrrr}17 & 1.203087 & -0.495824 & 3.036442 \\ 8 & 0.771900 & 1.740966 & 0.938183 \\ 8 & 1.039472 & -1.933815 & 0.301506 \\ 7 & 0.851052 & 0.258762 & -1.480015 \\ 7 & 2.899604 & 0.089818 & 0.457836 \\ 6 & 1.180395 & -2.641055 & -0.795421 \\ 6 & 0.849458 & -2.203366 & -2.025022 \\ 6 & 0.238595 & -0.862669 & -2.252814 \\ 1 & 0.293198 & -0.602801 & -3.330456 \\ 1 & -0.835602 & -0.873996 & -1.990907 \\ 6 & 0.452191 & 2.741879 & -1.158143 \\ 6 & 2.272348 & 0.404907 & -1.862506 \\ 1 & 2.366671 & 1.071861 & -2.741759 \\ 1 & 2.625460 & -0.593045 & -2.176725 \\ 6 & 0.055140 & 1.469494 & -1.825977 \\ 1 & -0.998734 & 1.219015 & -1.586124 \\ 1 & 0.093381 & 1.593907 & -2.927782 \\ 6 & 3.161390 & 0.890695 & -0.737078 \\ 1 & 2.951524 & 1.942793 & -0.497563 \\ 1 & 4.217877 & 0.835886 & -1.058994 \\ 6 & 0.730100 & 2.795256 & 0.157711\end{array}$


Complexes in Scheme S4

[ABP]CITiv $\left(\mathrm{OC}\left(\mathrm{OCH}_{3}\right) \mathrm{C}\left(\mathrm{CH}_{3}\right)_{2}\right)$ \& [ABP]ClTi"' $\left(\mathrm{OC}\left(\mathrm{OCH}_{3}\right) \mathrm{C}\left(\mathrm{CH}_{2}\right)\left(\mathrm{CH}_{3}\right)\right)$

$E=-4455.235673$

$G=-4454.603135$

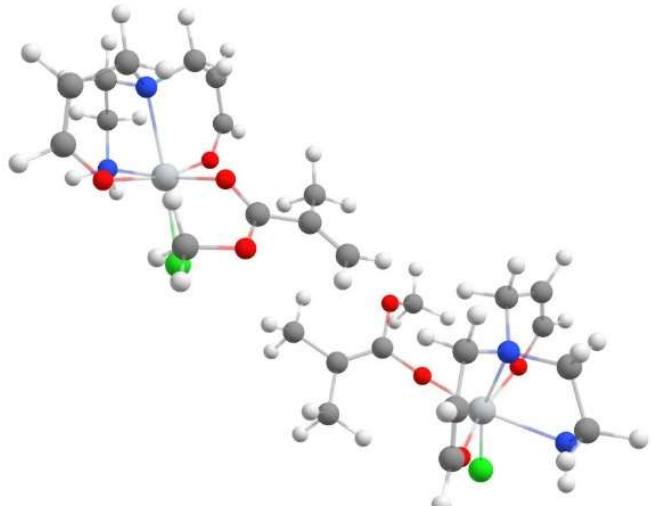

\begin{tabular}{rrrr}
17 & 6.843406 & -0.498147 & 2.347877 \\
8 & 5.369957 & 1.704586 & 0.786473 \\
8 & 5.890344 & -1.856953 & -0.277719 \\
7 & 4.735607 & 0.356076 & -1.626812 \\
7 & 7.360995 & 0.457420 & -0.613117 \\
6 & 5.739020 & -2.465565 & -1.429295 \\
6 & 4.911138 & -2.036548 & -2.402020 \\
6 & 4.049742 & -0.834019 & -2.215011 \\
1 & 3.607092 & -0.528288 & -3.185716 \\
1 & 3.201605 & -1.060314 & -1.540923 \\
6 & 4.120811 & 2.713726 & -0.921557 \\
6 & 5.859747 & 0.767732 & -2.494636 \\
1 & 5.510741 & 1.486487 & -3.261515 \\
1 & 6.198919 & -0.137294 & -3.027910 \\
6 & 3.695769 & 1.414996 & -1.516668 \\
1 & 2.882671 & 0.969586 & -0.913656 \\
1 & 3.268952 & 1.580271 & -2.528364 \\
6 & 7.039171 & 1.339339 & -1.734345 \\
1 & 6.804986 & 2.334933 & -1.333412 \\
1 & 7.889772 & 1.465889 & -2.429521 \\
6 & 4.871499 & 2.763581 & 0.195037 \\
22 & 5.519893 & -0.171329 & 0.532374 \\
6 & 1.737492 & 0.093088 & 1.941452 \\
6 & 0.302194 & -0.195739 & 2.223095 \\
1 & -0.051943 & -1.120865 & 1.748902 \\
1 & 0.134357 & -0.281736 & 3.312793 \\
1 & -0.358428 & 0.627480 & 1.894232 \\
6 & 2.286406 & 1.358465 & 2.512676 \\
1 & 3.370078 & 1.466847 & 2.360725 \\
1 & 1.782753 & 2.234902 & 2.060926 \\
1 & 2.079623 & 1.416765 & 3.596443 \\
6 & 2.522798 & -0.741183 & 1.221276 \\
8 & 3.825082 & -0.564239 & 1.052046 \\
8 & 2.010665 & -1.823864 & 0.566831 \\
6 & 2.683686 & -3.044247 & 0.800175 \\
1 & 3.736962 & -3.000892 & 0.474775 \\
1 & 2.658721 & -3.307579 & 1.873241 \\
1 & 2.157473 & -3.821581 & 0.229680 \\
1 & 3.746160 & 3.642717 & -1.358661 \\
1 & 5.132089 & 3.730889 & 0.656333 \\
1 & 4.843159 & -2.602131 & -3.334804 \\
1 & 6.348975 & -3.373770 & -1.571812 \\
1 & 7.829788 & -0.399476 & -0.916082 \\
& & & \\
\hline
\end{tabular}

$\begin{array}{rrrr}1 & 7.965173 & 0.909026 & 0.075253 \\ 17 & -3.296128 & 0.499641 & 2.236729 \\ 8 & -4.526298 & -1.825966 & 0.369604 \\ 8 & -5.664275 & 1.829894 & 0.529888 \\ 7 & -6.553200 & -0.380245 & -1.051616 \\ 7 & -6.581896 & -0.515541 & 1.831637 \\ 6 & -6.631268 & 2.471925 & -0.069941 \\ 6 & -7.231236 & 2.050974 & -1.203274 \\ 6 & -6.781531 & 0.817449 & -1.909885 \\ 1 & -7.514358 & 0.548481 & -2.700361 \\ 1 & -5.818408 & 0.987728 & -2.432124 \\ 6 & -5.788284 & -2.783844 & -1.353984 \\ 6 & -7.776403 & -0.727446 & -0.302046 \\ 1 & -8.420167 & -1.404985 & -0.898826 \\ 1 & -8.342053 & 0.211589 & -0.164024 \\ 6 & -6.108335 & -1.463239 & -1.968975 \\ 1 & -5.213067 & -1.052327 & -2.480303 \\ 1 & -6.882865 & -1.589756 & -2.755260 \\ 6 & -7.525030 & -1.338076 & 1.066588 \\ 1 & -7.094347 & -2.344288 & 0.966121 \\ 1 & -8.496752 & -1.454857 & 1.582980 \\ 6 & -4.985473 & -2.868094 & -0.273555 \\ 22 & -4.874539 & 0.062467 & 0.520425 \\ 6 & -1.078968 & -0.041377 & -0.956784 \\ 6 & 0.186744 & 0.380667 & -1.080741 \\ 1 & 0.436486 & 1.445710 & -1.064725 \\ 1 & 0.998640 & -0.347690 & -1.175103 \\ 6 & -1.486854 & -1.476381 & -0.925511 \\ 1 & -2.053335 & -1.690057 & -0.002321 \\ 1 & -2.164308 & -1.726992 & -1.760468 \\ 1 & -0.601405 & -2.126737 & -0.966265 \\ 6 & -2.173309 & 0.935218 & -0.801004 \\ 8 & -3.357912 & 0.603490 & -0.851915 \\ 8 & -1.807833 & 2.182751 & -0.620860 \\ 6 & -2.835178 & 3.126242 & -0.318572 \\ 1 & -3.590494 & 3.150347 & -1.119571 \\ 1 & -3.328167 & 2.846760 & 0.626032 \\ 1 & -2.337315 & 4.098626 & -0.230852 \\ 1 & -6.144816 & -3.696425 & -1.838818 \\ 1 & -4.705601 & -3.854762 & 0.137097 \\ 1 & -8.039836 & 2.645869 & -1.636297 \\ 1 & -6.966597 & 3.406922 & 0.415062 \\ 1 & -7.005685 & 0.359454 & 2.149141 \\ 1 & -6.231175 & -1.002339 & 2.657687\end{array}$

[ABP]CITiV $\left(\mathrm{OC}\left(\mathrm{OCH}_{3}\right) \mathrm{C}\left(\mathrm{CH}_{3}\right)_{2}\right)$ \& [ABP]CITi"' $\left(\mathrm{OC}\left(\mathrm{OCH}_{3}\right) \mathrm{C}\left(\mathrm{CH}_{2}\right)\left(\mathrm{CH}_{3}\right)\right)$ TS

$E=-4455.217415$

$G=-4454.578208$

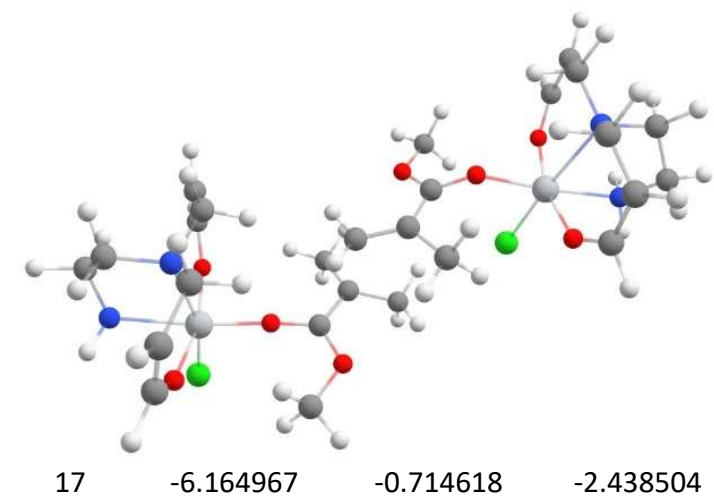




\begin{tabular}{|c|c|c|c|}
\hline 8 & -4.861574 & 1.685304 & -0.917051 \\
\hline 8 & -5.750067 & -1.660740 & 0.461484 \\
\hline 7 & -4.765702 & 0.652174 & 1.705908 \\
\hline 7 & -7.171053 & 0.684374 & 0.173938 \\
\hline 6 & -5.738346 & -2.175816 & 1.666771 \\
\hline 6 & -5.041292 & -1.656613 & 2.695931 \\
\hline 6 & -4.183651 & -0.449380 & 2.529155 \\
\hline 1 & -3.918606 & -0.034189 & 3.523180 \\
\hline 1 & -3.224590 & -0.707011 & 2.038902 \\
\hline 6 & -4.030677 & 2.938508 & 0.876389 \\
\hline 6 & -6.047995 & 1.102403 & 2.291509 \\
\hline 1 & -5.864963 & 1.856926 & 3.081804 \\
\hline 1 & -6.501956 & 0.226332 & 2.786877 \\
\hline 6 & -3.743813 & 1.736068 & 1.709323 \\
\hline 1 & -2.801428 & 1.259507 & 1.377015 \\
\hline 1 & -3.572728 & 2.036286 & 2.763565 \\
\hline 6 & -7.032288 & 1.641328 & 1.273451 \\
\hline 1 & -6.678271 & 2.593794 & 0.854998 \\
\hline 1 & -7.994941 & 1.846558 & 1.776851 \\
\hline 6 & -4.519403 & 2.831951 & -0.372258 \\
\hline 22 & -5.175909 & -0.118717 & -0.484508 \\
\hline 6 & -1.237973 & -0.308757 & -1.604684 \\
\hline 6 & -0.042896 & -0.929370 & -2.253636 \\
\hline 1 & 0.225165 & -1.896195 & -1.806287 \\
\hline 1 & -0.235043 & -1.095849 & -3.329464 \\
\hline 1 & 0.842911 & -0.274941 & -2.191139 \\
\hline 6 & -1.615180 & 1.061041 & -2.085468 \\
\hline 1 & -2.424756 & 1.511473 & -1.492207 \\
\hline 1 & -0.741774 & 1.731255 & -2.053992 \\
\hline 1 & -1.962550 & 1.023769 & -3.134598 \\
\hline 6 & -2.250344 & -1.134284 & -1.124835 \\
\hline 8 & -3.413491 & -0.707871 & -0.765037 \\
\hline 8 & -1.963852 & -2.421728 & -0.892812 \\
\hline 6 & -2.980551 & -3.258144 & -0.386957 \\
\hline 1 & -3.322722 & -2.931670 & 0.610804 \\
\hline 1 & -3.852184 & -3.287935 & -1.061664 \\
\hline 1 & -2.544251 & -4.261940 & -0.308664 \\
\hline 1 & -3.762902 & 3.923770 & 1.265830 \\
\hline 1 & -4.679776 & 3.722945 & -1.000293 \\
\hline 1 & -5.083934 & -2.144489 & 3.672827 \\
\hline 1 & -6.356479 & -3.078756 & 1.804100 \\
\hline 1 & -7.732085 & -0.129967 & 0.434912 \\
\hline 1 & -7.606409 & 1.101394 & -0.650440 \\
\hline 17 & 3.638110 & -0.340848 & -2.578975 \\
\hline 8 & 4.548002 & -1.892215 & 0.095277 \\
\hline 8 & 5.474743 & 1.687559 & -0.913784 \\
\hline 7 & 6.120314 & 0.118907 & 1.385990 \\
\hline 7 & 6.758320 & -0.798413 & -1.283102 \\
\hline 6 & 6.238199 & 2.581528 & -0.348180 \\
\hline 6 & 6.599468 & 2.563184 & 0.953013 \\
\hline 6 & 6.089117 & 1.521544 & 1.889737 \\
\hline 1 & 6.657535 & 1.562590 & 2.843817 \\
\hline 1 & 5.029695 & 1.709620 & 2.155820 \\
\hline 6 & 5.476710 & -2.190195 & 2.224220 \\
\hline 6 & 7.490771 & -0.267950 & 0.998634 \\
\hline 1 & 8.042972 & -0.671534 & 1.871864 \\
\hline 1 & 8.008938 & 0.659247 & 0.694620 \\
\hline 6 & 5.570865 & -0.723793 & 2.480918 \\
\hline 1 & 4.563040 & -0.306706 & 2.685040 \\
\hline 1 & 6.173891 & -0.536926 & 3.395291 \\
\hline 6 & 7.569709 & -1.257663 & -0.152614 \\
\hline 1 & 7.190691 & -2.241140 & 0.159439 \\
\hline 1 & 8.634237 & -1.393770 & -0.423585 \\
\hline
\end{tabular}

$\begin{array}{rrrr}6 & 4.929566 & -2.657361 & 1.082937 \\ 22 & 4.776586 & -0.080545 & -0.526598 \\ 6 & 0.838211 & -0.155956 & 0.517932 \\ 6 & -0.507250 & 0.127129 & 0.400243 \\ 1 & -0.830579 & 1.165108 & 0.273479 \\ 1 & -1.196651 & -0.529940 & 0.943496 \\ 6 & 1.353845 & -1.483248 & 0.978122 \\ 1 & 2.004927 & -1.949194 & 0.216543 \\ 1 & 1.977064 & -1.390472 & 1.887019 \\ 1 & 0.521523 & -2.171030 & 1.194354 \\ 6 & 1.827472 & 0.809581 & 0.141273 \\ 8 & 3.045913 & 0.636587 & 0.326492 \\ 8 & 1.388117 & 1.953001 & -0.382536 \\ 6 & 2.374044 & 2.858001 & -0.858985 \\ 1 & 3.068165 & 3.150883 & -0.054557 \\ 1 & 2.957227 & 2.395839 & -1.671222 \\ 1 & 1.831033 & 3.737320 & -1.226791 \\ 1 & 5.782587 & -2.888965 & 3.007268 \\ 1 & 4.810998 & -3.745281 & 0.928310 \\ 1 & 7.252139 & 3.350141 & 1.340514 \\ 1 & 6.611612 & 3.386365 & -1.008261 \\ 1 & 7.183661 & -0.003877 & -1.765960 \\ 1 & 6.607853 & -1.533552 & -1.975008\end{array}$

[ABP]CITi"l'OC(OCH$) \mathrm{C}\left(\mathrm{CH}_{3}\right)_{2} \mathrm{CH}_{2} \mathrm{C}\left(\mathrm{CH}_{3}\right) \mathrm{C}\left(\mathrm{OCH}_{3}\right) \mathrm{OT}$ $\mathrm{i}^{\mathrm{IV}}[\mathrm{ABP}] \mathrm{Cl}$
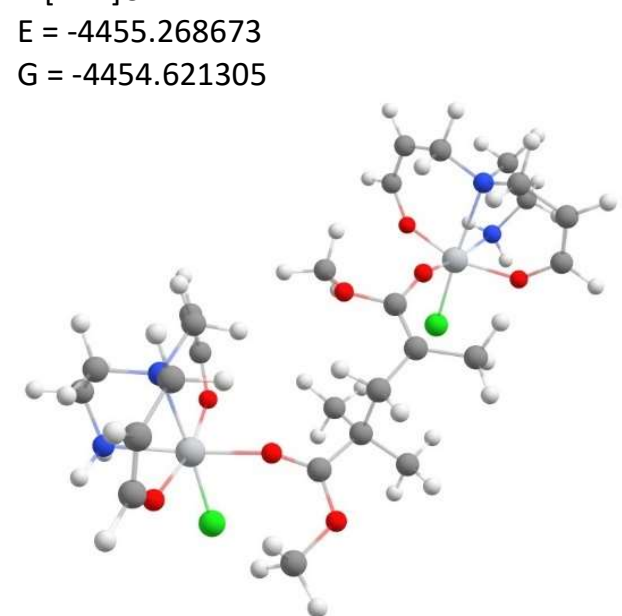

$\begin{array}{rrrr}17 & 5.217138 & 1.522943 & -2.147894 \\ 8 & 3.400021 & -1.082702 & -1.622219 \\ 8 & 5.995418 & 0.420598 & 0.770468 \\ 7 & 4.218792 & -1.792900 & 1.029565 \\ 7 & 6.172386 & -1.622260 & -1.119494 \\ 6 & 6.250634 & 0.191521 & 2.030067 \\ 6 & 5.500794 & -0.580295 & 2.846089 \\ 6 & 4.233652 & -1.234496 & 2.409868 \\ 1 & 3.974682 & -2.050514 & 3.119415 \\ 1 & 3.382277 & -0.525584 & 2.443266 \\ 6 & 2.549879 & -2.947620 & -0.499954 \\ 6 & 5.287030 & -2.795413 & 0.856932 \\ 1 & 4.949192 & -3.788575 & 1.219781 \\ 1 & 6.121842 & -2.487948 & 1.512252 \\ 6 & 2.857380 & -2.363694 & 0.838484 \\ 1 & 2.157495 & -1.528652 & 1.053617 \\ 1 & 2.687871 & -3.120598 & 1.633578 \\ 6 & 5.807977 & -2.930510 & -0.565922 \\ 1 & 5.036060 & -3.367640 & -1.215052 \\ 1 & 6.660394 & -3.636337 & -0.559654\end{array}$




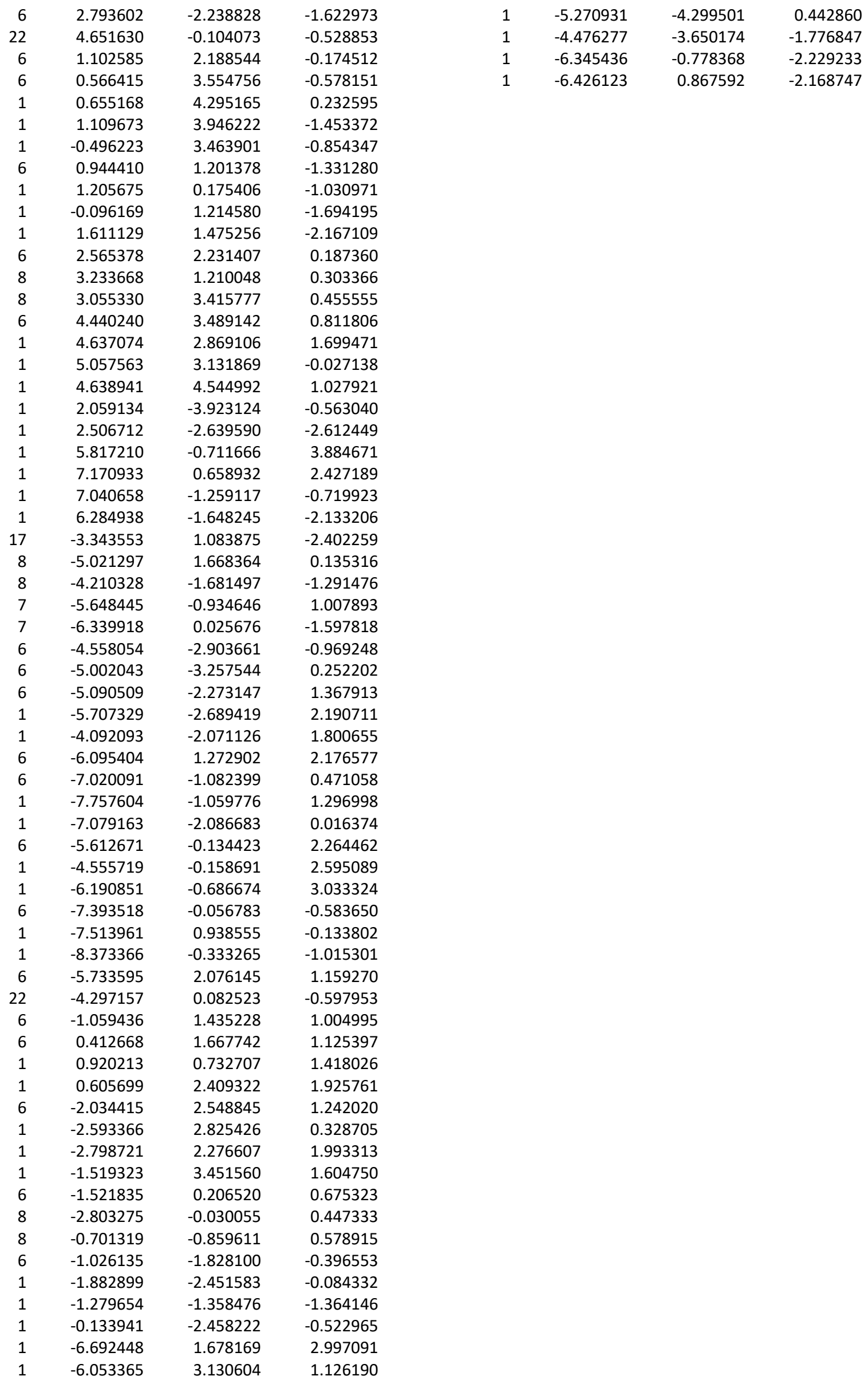




\section{Complexes in Scheme S5}

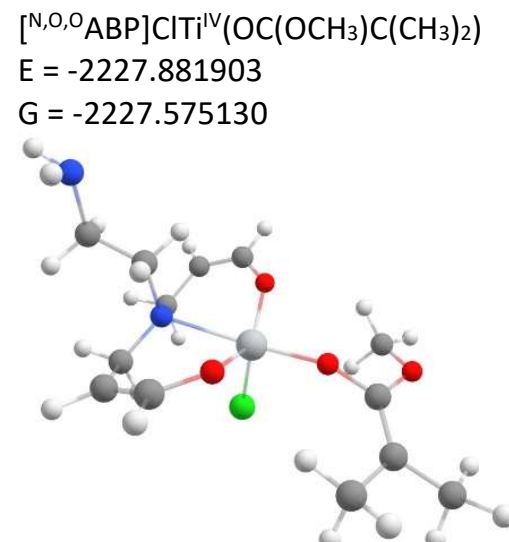

$\begin{array}{rrrr}17 & -0.729782 & 0.077164 & 2.152192 \\ 8 & 0.271185 & -1.649205 & -0.516428 \\ 8 & 0.423577 & 1.812969 & -0.467640 \\ 7 & 2.163248 & -0.020570 & 0.624713 \\ 7 & 4.978290 & -0.158120 & -1.877453 \\ 6 & 1.364946 & 2.699202 & -0.160322 \\ 6 & 2.354111 & 2.466380 & 0.715475 \\ 6 & 2.503872 & 1.167382 & 1.439072 \\ 1 & 3.536277 & 1.064632 & 1.829201 \\ 1 & 1.841866 & 1.136855 & 2.325306 \\ 6 & 2.066920 & -2.514021 & 0.748342 \\ 6 & 2.921635 & -0.060814 & -0.644427 \\ 1 & 2.644284 & 0.812265 & -1.253581 \\ 1 & 2.594542 & -0.952667 & -1.203685 \\ 6 & 2.387491 & -1.231782 & 1.445479 \\ 1 & 1.766781 & -1.116889 & 2.353055 \\ 1 & 3.437249 & -1.246900 & 1.800066 \\ 6 & 4.437575 & -0.086232 & -0.537471 \\ 1 & 4.760358 & -0.912497 & 0.135154 \\ 1 & 4.793450 & 0.851314 & -0.072515 \\ 6 & 1.074771 & -2.640309 & -0.146566 \\ 22 & -0.112412 & 0.097626 & -0.012004 \\ 6 & -3.855112 & -0.936270 & -0.366703 \\ 6 & -5.338626 & -0.876203 & -0.225405 \\ 1 & -5.662993 & -1.390426 & 0.697776 \\ 1 & -5.715873 & 0.154641 & -0.203778 \\ 1 & -5.837318 & -1.404537 & -1.059837 \\ 6 & -3.225621 & -2.285882 & -0.294138 \\ 1 & -3.431226 & -2.760317 & 0.683341 \\ 1 & -3.657360 & -2.960713 & -1.057106 \\ 1 & -2.137207 & -2.261314 & -0.442358 \\ 6 & -3.122389 & 0.182158 & -0.576580 \\ 8 & -1.796680 & 0.180528 & -0.651269 \\ 8 & -3.707274 & 1.389236 & -0.740636 \\ 6 & -3.044663 & 2.487966 & -0.159455 \\ 1 & -2.789817 & 2.292497 & 0.898976 \\ 1 & -2.117400 & 2.744168 & -0.699176 \\ 1 & -3.734787 & 3.341192 & -0.208668 \\ 1 & 2.653630 & -3.400985 & 1.002155 \\ 1 & 0.875180 & -3.602447 & -0.641529 \\ 1 & 3.070767 & 3.265933 & 0.920253 \\ 1 & 1.283621 & 3.660475 & -0.688553 \\ 1 & 5.992611 & -0.079367 & -1.870961 \\ 1 & 4.757844 & -1.058054 & -2.303773\end{array}$
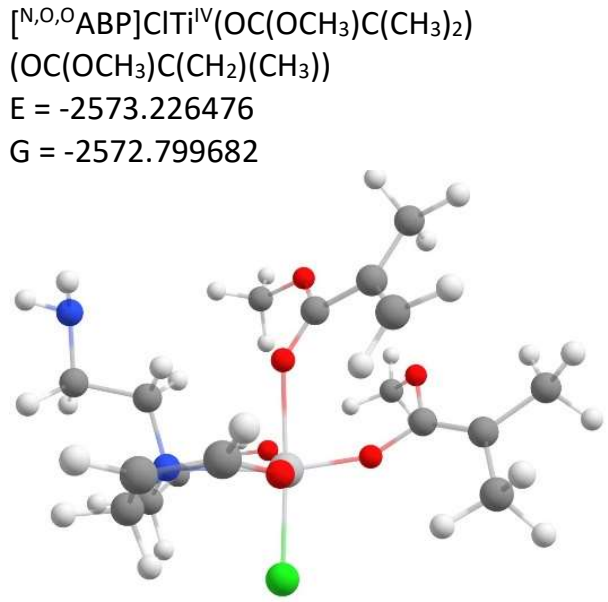

17

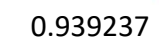

$-3.193534$

0.617748

8

$-1.212527$

$-1.647477$

0.835226

$-0.381820$

1.916684

2.594506

$-0.455830$

$-0.280118$

3.811887

3.075277

$-0.763775$

1.939396

$-0.175934$

2.606925

3.185697

3.476578

$-0.396565$

$-0.954592$

2.153859

4.532231

$-0.755331$

0.803039

3.370230

$-2.054242$

0.529021

2.213784

$-0.764370$

0.807817

2.670817

1.009892

$-2.732893$

2.245866

1.456494

$-0.408023$

2.007277

1.300984

0.501083

3.001857

$-1.154541$

$-1.239903$

2.874809

$-2.232981$

$-1.530151$

4.084433

$-0.998074$

$-1.320525$

4.031973

1.646638

$-1.702239$

4.555938

1.181394

$-0.628247$

4.676193

0.871283

1.481420

$-1.492526$

0.310671

$-0.845218$

0.253631

$-3.671741$

$-1.047124$

$-2.710060$

$-1.280300$

0.230383

$-5.063102$

$-5.799583$

$-0.741245$

$-0.216279$

$-0.233428$

$-5.203649$

$-5.330601$

$-1.53405$

$-0.006045$

0.078769

0.484767

$-3.369400$

$-0.363626$

$-1.239817$

$-2.317987$

$-2.422461$

$-1.128430$

$-1.072303$

$-0.897262$

$-3.005904$

$-3.296239$

$-2.179705$

$-2.708804$

$-1.463867$

2.153266

0.558577

0.613073

$-2.941045$

$-2.445543$

$-2.909666$

$-1.349673$

$-2.717673$

2.722573

0.268686

4.024485

1.782786

4.692752

3.336806

$-0.195455$

$-1.176972$

1.284241

0.393375

2.607457

3.205605

0.271101

2.638647

1.368243

3.047422

$-0.451045$

$-3.648247$

$-0.592633$

$-3.599218$

2.825707

3.623239

0.221853

$-0.788375$

$-1.644091$

$-0.226273$

$-1.079402$

3.275678

$-0.240083$

1.962723

3.031542

0.537319 


$\begin{array}{lrrr}6 & 0.124947 & 3.151706 & 1.384199 \\ 1 & 0.294676 & 2.217922 & 1.941578 \\ 1 & 1.026085 & 3.379616 & 0.790430 \\ 1 & -0.086682 & 3.979501 & 2.070774 \\ 6 & -2.268700 & 1.955951 & -1.115250 \\ 6 & -2.363504 & 0.969408 & -2.017786 \\ 1 & -1.598232 & 0.191021 & -2.096631 \\ 1 & -3.226929 & 0.913431 & -2.688462 \\ 6 & -3.303540 & 3.015355 & -0.921033 \\ 1 & -2.876191 & 4.026996 & -1.010761 \\ 1 & -4.111719 & 2.906226 & -1.658712 \\ 1 & -3.744627 & 2.944745 & 0.087326\end{array}$
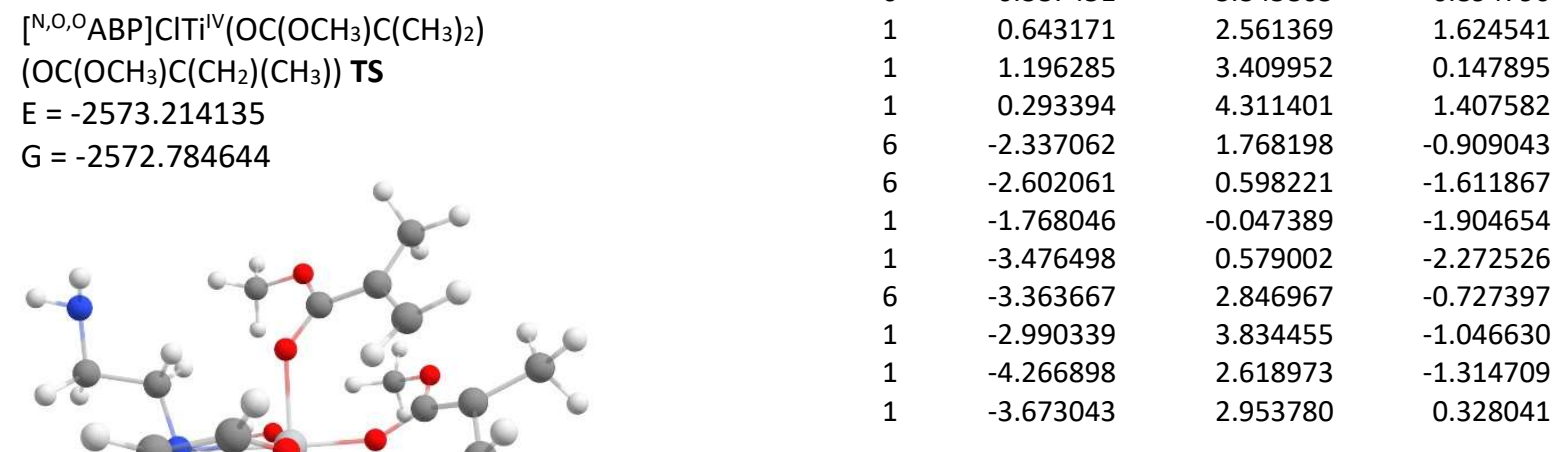

$\left[{ }^{\mathrm{N}, \mathrm{O}, \mathrm{O}} \mathrm{ABP}\right] \mathrm{ClTi}{ }^{\mathrm{iV}}\left(\mathrm{OC}\left(\mathrm{OCH}_{3}\right) \mathrm{C}\left(\mathrm{CH}_{3}\right) \mathrm{CH}_{2} \mathrm{C}\left(\mathrm{CH}_{3}\right)_{2} \mathrm{COOC}\right.$ $\left.\mathrm{H}_{3}\right)$

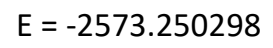

0.573518

2.555513

$-0.188284$

$-343578$

$0.690312 \quad 2.353018$

$1.525480 \quad 2.904225$

$-0.831918-3.574215$

$-0.888270 \quad-3.511715$

$-0.017412 \quad 2.848028$

$0.560508 \quad 3.593890$

$3.277719-1.249460$

$2.978617 \quad-2.009535$

$1.073282-0.315735$

$1.912373-0.307143$

$3.093053 \quad 0.291280$

$3.345865 \quad 0.894790$

$2.561369 \quad 1.624541$

$3.409952 \quad 0.147895$

$4.311401 \quad 1.407582$

$1.768198-0.909043$

$0.598221-1.611867$

$-1.904654$

$2.846967-0.727397$

$-1.314709$

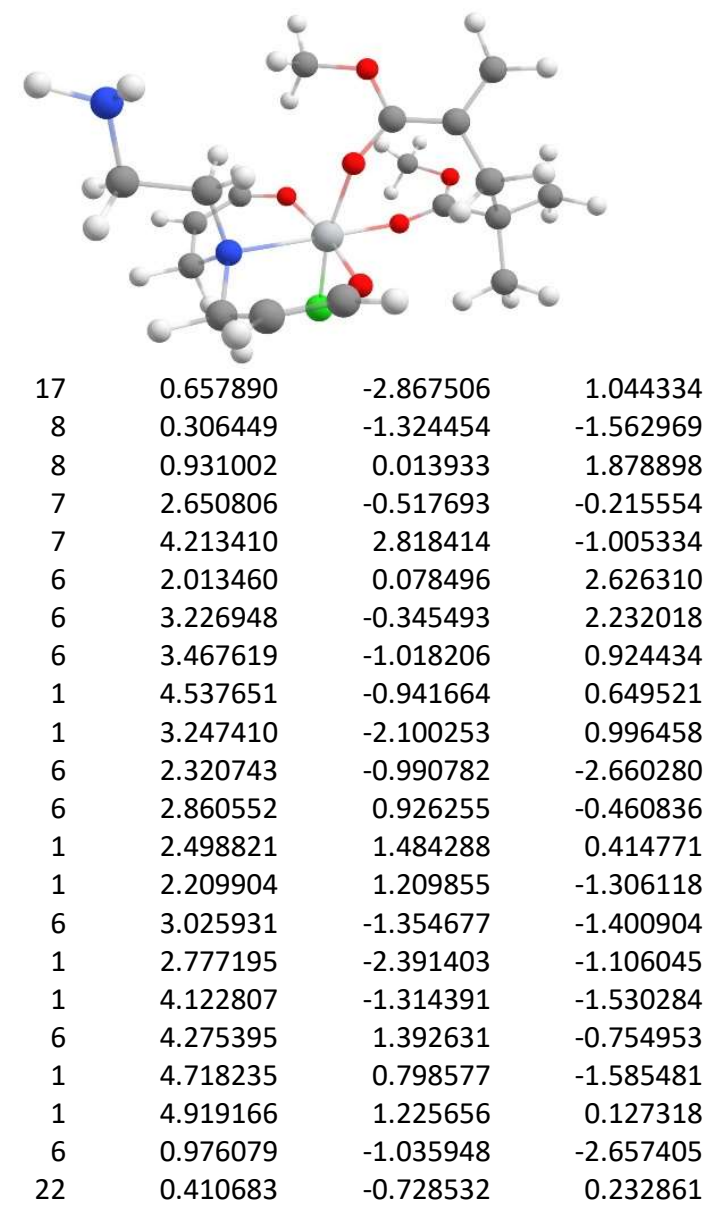




$\begin{array}{rrrr}6 & -3.412181 & -0.733966 & -0.825246 \\ 6 & -4.915821 & -0.500149 & -0.766058 \\ 1 & -5.415500 & -1.232441 & -0.111083 \\ 1 & -5.171454 & 0.506222 & -0.404050 \\ 1 & -5.335482 & -0.613216 & -1.779073 \\ 6 & -3.111000 & -2.107947 & -1.420559 \\ 1 & -2.031267 & -2.325054 & -1.416195 \\ 1 & -3.635707 & -2.910565 & -0.875895 \\ 1 & -3.459224 & -2.128226 & -2.466665 \\ 6 & -2.762881 & -0.616678 & 0.533354 \\ 8 & -1.597761 & -0.940064 & 0.748190 \\ 8 & -3.504551 & -0.130806 & 1.498703 \\ 6 & -2.826567 & 0.193272 & 2.713064 \\ 1 & -2.418858 & -0.713450 & 3.183202 \\ 1 & -1.995560 & 0.886043 & 2.502488 \\ 1 & -3.570611 & 0.665185 & 3.363901 \\ 1 & 2.875626 & -0.733573 & -3.565657 \\ 1 & 0.372641 & -0.824122 & -3.555507 \\ 1 & 4.069594 & -0.236902 & 2.919797 \\ 1 & 1.875694 & 0.547454 & 3.613660 \\ 1 & 5.144051 & 3.219252 & -1.098696 \\ 1 & 3.724483 & 3.004555 & -1.880957 \\ 8 & -0.220222 & 0.984559 & -0.343781 \\ 6 & -1.213909 & 1.830792 & -0.303781 \\ 8 & -1.021258 & 2.968018 & 0.412545 \\ 6 & 0.258047 & 3.217765 & 0.940934 \\ 1 & 0.572568 & 2.429057 & 1.645223 \\ 1 & 1.027638 & 3.307705 & 0.153224 \\ 1 & 0.192243 & 4.175440 & 1.474951 \\ 6 & -2.431004 & 1.643596 & -0.890542 \\ 6 & -2.699753 & 0.377060 & -1.655239 \\ 1 & -1.749899 & -0.035809 & -2.030962 \\ 1 & -3.336855 & 0.581240 & -2.537486 \\ 6 & -3.511566 & 2.665164 & -0.708647 \\ 1 & -3.106940 & 3.689531 & -0.716304 \\ 1 & -4.266750 & 2.590432 & -1.509236 \\ 1 & -4.050100 & 2.554252 & 0.254086\end{array}$

$\left[{ }^{\mathrm{N}, \mathrm{N}, \mathrm{O}, \mathrm{O}} \mathrm{ABP}\right] \mathrm{CITi \textrm {iV }}\left(\mathrm{OC}\left(\mathrm{OCH}_{3}\right) \mathrm{C}\left(\mathrm{CH}_{3}\right) \mathrm{CH}_{2} \mathrm{C}\left(\mathrm{CH}_{3}\right)_{2} \mathrm{COO}\right.$ $\left.\mathrm{CH}_{3}\right)$

$E=-2573.272045$

$G=-2572.838379$

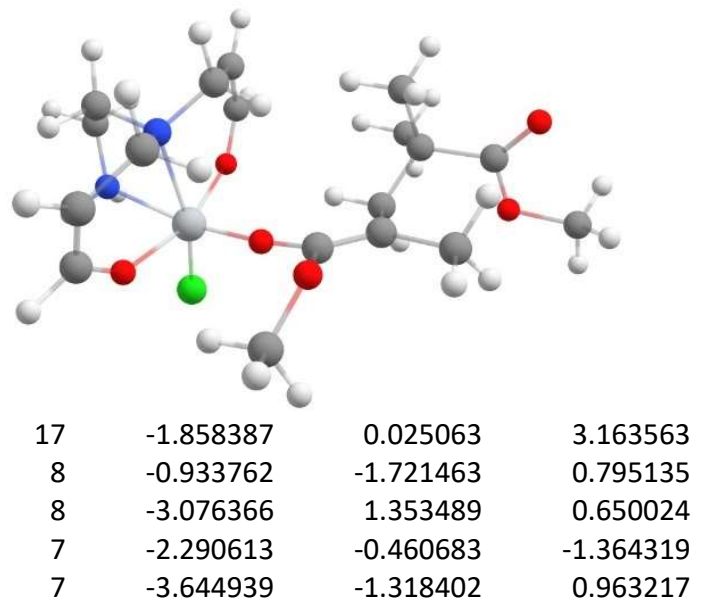

\begin{tabular}{|c|c|c|}
\hline-3.763743 & 1.923021 & -0.312658 \\
\hline-3.539098 & 1.714513 & -1.623734 \\
\hline-2.443758 & 0.826632 & -2.107555 \\
\hline-2.601713 & 0.584108 & -3.178810 \\
\hline-1.463381 & 1.336046 & -2.046939 \\
\hline-0.762357 & -2.480969 & -1.414670 \\
\hline-3.540068 & -1.246771 & -1.461384 \\
\hline-3.538007 & -1.860070 & -2.383902 \\
\hline-4.366580 & -0.521210 & -1.559089 \\
\hline-1.140225 & -1.161877 & -1.998402 \\
\hline-0.286728 & -0.455089 & -1.941665 \\
\hline-1.367140 & -1.276633 & -3.078086 \\
\hline-3.811085 & -2.116893 & -0.251068 \\
\hline-3.107268 & -2.959610 & -0.206041 \\
\hline-4.825483 & -2.548737 & -0.336580 \\
\hline-0.641429 & -2.648974 & -0.083728 \\
\hline-1.795020 & -0.028697 & 0.896293 \\
\hline 1.957725 & 1.238448 & -0.049691 \\
\hline 3.054905 & 2.116680 & -0.558460 \\
\hline 2.681162 & 3.100634 & -0.871427 \\
\hline 3.822073 & 2.263188 & 0.223873 \\
\hline 3.581059 & 1.674205 & -1.423888 \\
\hline 2.322529 & -0.047304 & 0.628181 \\
\hline 1.407618 & -0.616675 & 0.866370 \\
\hline 2.811396 & 0.171849 & 1.597123 \\
\hline 0.661051 & 1.616304 & -0.140259 \\
\hline-0.340491 & 0.945663 & 0.406213 \\
\hline 0.292393 & 2.731305 & -0.825397 \\
\hline-0.483781 & 3.659401 & -0.096085 \\
\hline-1.428166 & 3.216707 & 0.261578 \\
\hline 0.079312 & 4.040241 & 0.776095 \\
\hline-0.709042 & 4.497195 & -0.769678 \\
\hline-0.502051 & -3.303229 & -2.086048 \\
\hline-0.307072 & -3.613858 & 0.335216 \\
\hline-4.160925 & 2.233347 & -2.357752 \\
\hline-4.569091 & 2.600482 & 0.017442 \\
\hline-4.423948 & -0.673832 & 1.115228 \\
\hline-3.559191 & -1.896817 & 1.800319 \\
\hline 3.255263 & -0.987383 & -0.165972 \\
\hline 2.709793 & -1.261715 & -1.558861 \\
\hline 2.640683 & -0.343692 & -2.163613 \\
\hline 3.358071 & -1.965348 & -2.103942 \\
\hline 1.702229 & -1.702287 & -1.477486 \\
\hline 3.367705 & -2.307382 & 0.605809 \\
\hline 4.072987 & -2.998549 & 0.113913 \\
\hline 3.708058 & -2.141806 & 1.639991 \\
\hline 2.379380 & -2.796477 & 0.641441 \\
\hline 4.664993 & -0.429587 & -0.284446 \\
\hline 5.321708 & -0.384478 & -1.292738 \\
\hline 5.144639 & -0.022882 & 0.903756 \\
\hline 6.456000 & 0.504526 & 0.892748 \\
\hline 7.179006 & -0.230600 & 0.506362 \\
\hline 6.511392 & 1.401829 & 0.254756 \\
\hline 6.701753 & 0.766451 & 1.929393 \\
\hline
\end{tabular}




\section{NOTES AND REFERENCES}

(1) Min, K.; Gao, H.; Yoon, J. A.; Wu, W.; Kowalewski, T.; Matyjaszewski, K. One-Pot Synthesis of Hairy Nanoparticles by Emulsion ATRP. Macromolecules 2009, 42 (5), 1597-1603.

(2) Penzel, E.; Goetz, N. Solution Properties of Polyacrylic Esters. Die Angew. Makromol. Chemie 1990, 178 (1), 191-200.

(3) Dwyer, A. B.; Chambon, P.; Town, A.; He, T.; Owen, A.; Rannard, S. P. Is Methanol Really a Bad Solvent for Poly(n-Butyl Methacrylate)? Low Dispersity and High Molecular Weight Polymers of n-Butyl Methacrylate Synthesised via ATRP in Anhydrous Methanol. Polym. Chem. 2014, 5 (11), 3608-3616.

(4) Grcev, S.; Schoenmakers, P.; ledema, P. Determination of Molecular Weight and Size Distribution and Branching Characteristics of PVAc by Means of Size Exclusion Chromatography/Multi-Angle Laser Light Scattering (SEC/MALLS). Polymer (Guildf). 2004, 45 (1), 39-48.

(5) Evans, D. F. The Determination of the Paramagnetic Susceptibility of Substances in Solution by Nuclear Magnetic Resonance. J. Chem. Soc. 1959, 2003-2005.

(6) Dolomanov, O. V.; Bourhis, L. J.; Gildea, R. J.; Howard, J. A. K.; Puschmann, H. OLEX2: A Complete Structure Solution, Refinement and Analysis Program. J. Appl. Crystallogr. 2009, 42 (2), 339-341.

(7) Sheldrick, G. M. Crystal Structure Refinement with SHELXL. Acta Crystallogr. Sect. C Struct. Chem. 2015, $71,3-8$.

(8) Sheldrick, G. M. A Short History of SHELX. Acta Crystallogr. Sect. A Found. Crystallogr. 2008, 64 (1), 112-122.

(9) Frisch, M. J.; Trucks, G. W.; Schlegel, H. B.; Scuseria, G. E.; Robb, M. A.; Cheeseman, J. R.; Scalmani, G.; Barone, V.; Mennucci, B.; Petersson, G. A.; et al. Gaussian 09, Revision E.01. Gaussian 09, Revision E.01, Gaussian, Inc. Wallingford CT 2013.

(10) Barroso, S.; Cui, J.; Carretas, J. M.; Cruz, A.; Santos, I. C.; Duarte, M. T.; Telo, J. P.; Marques, N.; Martins, A. M. Diamine Bis(Phenolate) M(III) (Y, Ti) Complexes: Synthesis, Structures, and Reactivity. Organometallics 2009, 28 (12), 3449-3458.

(11) Repeated Elemental Analysis of This Sample Consistently Gave Lower than Expected C,H, N Compositions. We Ascribe This to Oxidative Decomposition of the (Air-Sensitive) Sample Either during Transit or Analytical Sample Preparation (or Both).

(12) Johnson, A. L.; Davidson, M. G.; Mahon, M. F. Reactivity of Boranes with a Titanium(Iv) Amine Tris(Phenolate) Alkoxide Complex; Formation of a Ti(Iv) Tetrahydroborate Complex, a Ti(lii) Dimer and a Ti(Iv) Hydroxide Lewis Acid Adduct. Dalt. Trans. 2007, No. 46, 5405. 